NBER WORKING PAPER SERIES

\title{
WHAT CAN RATIONAL INVESTORS DO ABOUT EXCESSIVE VOLATILITY AND SENTIMENT FLUCTUATIONS?
}

\author{
Bernard Dumas \\ Alexander Kurshev \\ Raman Uppal \\ Working Paper 11803 \\ http://www.nber.org/papers/w11803 \\ NATIONAL BUREAU OF ECONOMIC RESEARCH
1050 Massachusetts Avenue
Cambridge, MA 02138
November 2005
}

We are grateful to Wei Xiong for extensive discussions. We are also grateful for comments from Andrew Abel, Suleyman Basak, Tomas Björk, Andrea Buraschi, Joao Cocco, John Cotter, Xavier Gabaix, Francisco Gomes, Joao Gomes, Tim Johnson, Leonid Kogan, Kostas Koufopoulos, Karen Lewis, Deborah Lucas, Pascal Maenhout, Massimo Massa, Stavros Panageas, Anna Pavlova, Ludovic Phalippou, Valery Polkovnichencko, Bryan Routledge, Andrew Scott, Alex Stomper, Allan Timmermann, Skander van den Heuvel, Luis Viceira, Pierre-Olivier Weill, Hongjun Yan, Amir Yaron, Moto Yogo, Joseph Zechner, Stanley Zin and participants in workshops held at Bank of England, INSEAD, London Business School, Massachusetts Institute of Technology, University of Vienna, Wharton School, University College Dublin, University of Piraeus, the European Summer Symposium in Financial Markets at Gerzensee, and the NBER "Capital Markets and the Economy" workshop. The views expressed herein are those of the author(s) and do not necessarily reflect the views of the National Bureau of Economic Research.

(C2005 by Bernard Dumas, Alexander Kurshev, and Raman Uppal. All rights reserved. Short sections of text, not to exceed two paragraphs, may be quoted without explicit permission provided that full credit, including (C) notice, is given to the source. 
What Can Rational Investors Do About Excessive Volatility and Sentiment Fluctuations?

Bernard Dumas, Alexander Kurshev, and Raman Uppal

NBER Working Paper No. 11803

November 2005

JEL No. G1

\section{$\underline{\text { ABSTRACT }}$}

Our objective is to understand the trading strategy that would allow an investor to take advantage of "excessive" stock price volatility and "sentiment" fluctuations. We construct a general equilibrium model of sentiment. In it, there are two classes of agents and stock prices are excessively volatile because one class is overconfident about a public signal. This class of irrational agents changes its expectations too often, sometimes being excessively optimistic, sometimes being excessively pessimistic. We find that because irrational traders introduce an additional source of risk, rational investors reduce the proportion of wealth invested into equity except when they are extremely optimistic about future growth. Moreover, their optimal portfolio strategy is based not just on a current price divergence but also on a prediction concerning the speed of convergence. Thus, the portfolio strategy includes a protection in case there is a deviation from that prediction. We find that long maturity bonds are an essential accompaniment of equity investment, as they serve to hedge this "sentiment risk." The answer to the question posed in the title is: "There is little that rational investors can do optimally to exploit, and hence, eliminate excessive volatility, except in the very long run."

Bernard Dumas

Department of Finance

INSEAD

77305 Fontainebleau Cedex

FRANCE

and NBER

bernard.dumas@insead.edu

Alexander Kurshev

London Business School

akurshev.phd2003@1ondon.edu

Raman Uppal

London Business School

ruppal@london.edu 


\section{Introduction}

Suppose that a Bayesian, intertemporally optimizing investor operates in a financial market that is deemed to have fluctuations in market sentiment and asset returns that are "excessively volatile." We would like to know what investment policy this person will undertake under market equilibrium, what effect his or her intervention will have on equilibrium prices, and whether he or she will ultimately eradicate the source of excess volatility.

To address these issues, we need a model of a financial market in which a subpopulation of investors trades on sentiment and generates excess volatility and we need to specify the rules of the contest between these investors and the rational investors.

We construct a model where some investors are non-Bayesian in the sense that they give too much credence to some public information signal. One way to capture that behavioral feature has recently been proposed by Scheinkman and Xiong (2003). In their model of a "tree" economy, a stream of dividends is paid. Some aspect of the stochastic process of dividends is not observable by anyone. All investors are risk neutral, are constrained from short selling, and receive information in the form of the current dividend and some public signals. Rational agents know the true correlation between innovations in the signal and innovations in the unobserved variables. Irrational (they call them "overconfident") agents are people who steadfastly believe that this correlation is a positive number when, in fact, it is zero. This causes them to give too much weight to the signals. Thus, when they receive a signal, they overreact to it, which then generates excessive stock price movements.

Here, we consider a setting similar to that in Scheinkman and Xiong (2003) except that all investors are risk averse (and are allowed to sell short) and only one group of agents is overconfident while the other is rational. Because some investors in our model are overconfident about the public signal, they are also fickle and change their beliefs too often about economic prospects. This is the source of excessive volatility. We refer to "excessive volatility" as a situation in which, for the given utility functions of agents, the level of volatility is larger than it would if all agents were rational Bayesians, and we refer to the fluctuations in the probability beliefs of irrational agents relative to those of rational agents as fluctuations in "sentiment."

In their contest with rational investors, we want the traders who are irrational in the way they form their beliefs, to still be full-fledged intertemporal optimizers. It is well-known that complete irrationality in the manner of positive "feedback traders" à la De Long, Shleifer, Summers, and Waldmann (1990b) can amplify the volatility of stock prices and that the additional volatility creates "noise-trader risk" for rational arbitrageurs, thereby creating a limit to arbitrage. However, feedback traders or traders acting randomly may not be the best representation of irrational behavior as they constitute excessively easy game for rational investors. For this reason, we prefer to model a general equilibrium economy where the irrational traders are intertemporal optimizers, even if they are non-Bayesian in their learning. In this way, welfare analysis and the analysis of gains and losses of the two categories of traders remain meaningful. ${ }^{1}$

Our main goal is to derive and analyze equilibrium prices and the optimal dynamic trading strategy of the rational investors. We identify three distinctive aspects of the portfolio strategy adopted by rational investors. First, these investors may not agree today with the market about its current estimate of the growth rate of dividends: there is dispersion of beliefs. When the rational investors are more optimistic than the market, they increase their investment in equity while decreasing their investment in bonds, because equity and bonds are positively correlated. Second, if there is disagreement today, rational investors are aware that irrational investors will revise their estimate differently from the way their own estimate is revised: disagreement drives sentiment. Third, even if the two groups of investors

\footnotetext{
${ }^{1}$ Furthermore, models of feedback trading do not discuss the budget constraint of the feedback traders and, therefore, leave unclear the origin of the gains that the rational arbitrageurs would make at their expense. And, even when noise traders pursue an explicit objective, as is done in De Long, Shleifer, Summers, and Waldmann (1990a), one must be careful not to confuse "noise risk" with some output risk induced by the noise risk, as has been pointed out recently by Loewenstein and Willard (2005). Restricting our analysis to a pure exchange general equilibrium economy allows us to maintain a clean distinction between output risk and noise risk.
} 
happen to agree today, investors are aware that next period they will all revise their estimates. The second and third effects cause rational investors to hedge: they hold fewer shares of equity than would be optimal in a market without excess volatility and they take a positive position in bonds (which would be zero in the absence of excess volatility).

Overall, rational risk arbitrageurs find it beneficial to trade on their belief that the market is being foolish but, when doing so, must hedge future fluctuations in the market's sentiment. Thus, our analysis illustrates how "risk arbitrage" must be based not just on a current price divergence but also on a model of irrational behavior and a prediction concerning the dynamics of market sentiment. And, the risk arbitrage must include a protection in case there is a deviation from that prediction.

The profitability of the rational "risk arbitrage" strategy and the survival time of irrational investors are two sides of the same coin. We derive the speed of impoverishment of the irrational traders, or the speed of enrichment of the rational ones. Previous work (Kogan, Ross, Wang, and Westerfield (2003); Yan (2004)) has examined the survival of traders who are permanently optimistic or pessimistic. Here, we study the survival of traders who are sometimes optimistic and sometimes pessimistic, depending on the sequence of signals they have received. We find that, in contrast to what is typically assumed in standard models of asset pricing, in our model the presence of a few rational traders is not sufficient to eliminate the effect of overconfident investors on excess volatility, and that even a moderate-sized group of overconfident investors may do a lot of damage and survive for a long time before being driven out of the market by rational investors.

Our model can be viewed as an equilibrium model of investor sentiment, in the sense of Barberis, Shleifer, and Vishny (1998). Several authors have supported the hypothesis that agents active in the financial markets exhibit aspects of behavior that deviate from rationality. This was done typically on the basis of some natural experiments, for instance, spin offs, share repurchases, initial public offerings, reactions to news, etc. A gamut of behavioral aspects have been suggested, not all of which are consistent with each other. We do not know which behavioral aspects actually prevail in the financial markets and which do not. In order to sort this out, it would be important to conduct tests using data on asset prices. To that aim, one must deduce theoretically the behavior of asset prices and portfolio choices that will prevail in the financial markets as a result of a particular deviation from rationality. Indeed, if one could then establish empirically that asset prices and portfolio choices deviate from market efficiency precisely in the manner predicted by the theory, one would be able to distinguish between behavioral aspects that are not actually present from those that have a decisive impact on financial choices and equilibria. That was the intent of three classic papers in this strand of the literature, which can be called "behavioral equilibrium theory:" Barberis, Shleifer, and Vishny (1998), Daniel, Hirshleifer, and Subrahmanyam (1998), and Hong and Stein (1999). The first two of these papers feature a single group of agents who are non-Bayesian. ${ }^{2}$ The model of Hong and Stein (1999), like ours, features two categories of agents with heterogeneous beliefs who, however, are not intertemporal optimizers.

The balance of this paper covers the following material. Section 2 reviews additional literature that is related to our work. In Section 3, we present our modeling choices for the economy. In Section 4, we determine the equilibrium in this economy. In Section 5, we discuss the impact of irrational traders on asset prices, return volatilities, and risk premia and we analyze the proportion of rational investors needed in the economy to reduce the excessive volatility. In Section 6, we identify the main factors driving the portfolio strategy of the rational trader. In Section 7, we discuss the survival of irrational traders over time and the profits made at their expense by the rational ones. Section 8 contains the conclusion. We highlight our main results in propositions, while all the mathematical derivations are collected in appendixes.

\footnotetext{
${ }^{2}$ The agents in Barberis, Shleifer, and Vishny (1998) update their beliefs using Bayes' formula but they do so on the basis of the wrong prior category of models, which they refuse to update.
} 


\section{Related work}

Our paper is connected to at least five strands of literature, which are not completely separate from each other. The first one - "behavioral equilibrium theory" - has already been referred to in the introduction. The second one is the strand of literature that deals with heterogeneous beliefs in financial-market equilibrium. ${ }^{3}$ Heterogeneity of beliefs between agents needs to be sustained or it quickly become irrelevant. There exist basically three ways of doing that. Differences in the basic model agents believe in, or in some fixed model parameter was proposed earlier by Harris and Raviv (1993), Kandel and Pearson (1995) and Cecchetti, Lam, and Mark (2000) and used more recently by David (2004). Under this approach, agents are non-Bayesian. Another modeling possibility is differences in priors, while agents remain Bayesian, as in Biais and Bossaerts (1998), Buraschi and Jiltsov (2005), Detemple and Murthy (1994), Gallmeyer (2000), and Duffie, Garleanu, and Pedersen (2002). The model we use allows for both differences in priors and also differences in the model agents believe in. ${ }^{4}$ A third, more sophisticated possibility that, however, includes traders that behave randomly, is to let agents receive private signals as in the vast "Noisy-Rational Expectations" literature originating from the work of Grossman and Stiglitz (1980), Hellwig (1980) and Wang (1993). In the case of private signals, agents also learn from price, a channel that is not present in our model.

Thirdly, our paper is motivated by the empirical work that has been conducted on the excess volatility puzzle of Shiller (1981) and LeRoy and Porter (1981). ${ }^{5}$ The excess volatility puzzle is that "stock prices move too much to be justified by changes in subsequent dividends." ${ }^{6}$ In deriving their bounds, Shiller (1981) and LeRoy and Porter (1981) had made the assumption that discount rates, by which future dividends are discounted to obtain the current price, were constant into the future, thereby overstating the degree to which the theoretical volatility was smaller than the observed one. Here, we use the time-varying, equilibrium discount factors that derive from intertemporal optimization with learning.

The excess-volatility puzzle is one aspect of market inefficiency that begs for a behavioral explanation. It is natural to ascribe the excess volatility to fluctuations in irrational "sentiment". But Shiller never indicated the way in which rational investors could cause those responsible for excess volatility to part with their wealth. The volatility of stock prices, if it is excessive relative to the volatility of fundamentals, is an indication that the financial market is not information efficient. If so, there must exist a trading strategy that allows a rational, intertemporally optimizing investor (a "risk arbitrageur") to take advantage of this inefficiency. The main goal of our paper is to calculate and understand the strategy of rational investors and the impact of this on irrational investors responsible for excess volatility. ${ }^{7}$

Some headway into the design of a portfolio strategy has already been made in past research which dealt with the logical link that exists between the phenomenon of excessive volatility and the predictability of stock returns. ${ }^{8}$ Campbell and Shiller (1988a,b) and Cochrane (2001, page 394 ff), have pointed out

\footnotetext{
${ }^{3}$ For a comprehensive study of the influence of heterogeneous beliefs on asset prices, see Basak (2004). For a survey of the behavioral view of asset pricing, see Shefrin (2005).

${ }^{4}$ Heterogeneity of beliefs opens the possibility that investors will engage in "speculative behavior" in the sense of Harrison and Kreps (1978). We return to that topic in Subsection 5.1.

${ }^{5}$ We must underscore that our definition of excessive volatility, given in the introductory section above, is not identical to that of Shiller (1981).

${ }^{6} \mathrm{~A}$ controversy about excess volatility has been going on since the publication of Shiller (1981) and the matter is not fully settled even today. The empirical method of Shiller has been criticized. Flavin (1983) and Kleidon (1986) have pointed out that stock prices and dividends could not be detrended by a deterministic trend based on realized returns, as Shiller had done. Furthermore, if the process for prices and/or dividends is not stationary, the ergodic theorem does not apply and volatility, defined originally across the possible sample paths, cannot be measured over time. Even in the case of stationarity, a near-unit root may exist in the behavior of these two variables, causing the statistic to reject Shiller's variance inequality in finite samples when it should not be rejected. Good methodological evaluations are provided by West (1988a,b) and Cochrane (1991).

${ }^{7}$ The question being answered in our paper is the same as the one raised by Williams (1977) and Ziegler (2000) in a simpler setting in which the expected growth rate of dividends is constant (although unobserved) and in which there are fewer securities. In these two papers, the investor whose strategy one is studying is assumed to be of negligible weight in the market, in contrast to our model. For a review of the literature on models with incomplete information, see Feldman (2005).

${ }^{8}$ That relation is analogous to the relation established by Froot and Frankel (1989) between variance-bounds tests à la Shiller and regression tests of predictability.
} 
that the dividend-price ratio would be constant over time if dividends were unpredictable (specifically, if they followed a geometric Brownian walk) and expected returns were constant. Since the dividend-price ratio is changing, its changes must be predicting either future changes in dividends or future changes in expected returns. This statement is true in any economic model, unless there are violations of the transversality conditions. Empirically, the dividend-price ratio hardly predicts subsequent dividends. It must, therefore, predict returns. But, if it predicts returns, it can serve as valuable information for a rational trader, or arbitrageur, entering the market. That aspect is embedded in our model.

Fourthly, our model can be compared to other models that account for excessive volatility. In the literature on excess volatility, there are at least three kinds of models not based on differences in beliefs that have been considered. One class of models shows that Bayesian, rational learning alone can serve to develop theoretical models with volatility that matches the data, by assuming that investors do not know the true stochastic process of dividends. For instance, Barsky and De Long (1993) write that: "Major long-run swings in the U.S. stock market over the past century are broadly consistent with a model driven by changes in current and expected future dividends in which investors must estimate the time-varying long-run dividend growth rate" [our emphasis]. As investors do not know the expected growth rate of dividends, prices are revised when they receive information about it. These price revisions go beyond the change in the current dividend because the current dividend also contains information about future dividends. A similar argument has been made by Timmermann $(1993,1996)$, Bullard and Duffy (1998), David and Veronesi (2002), and Veronesi (1999). Brennan and Xia (2001) calibrate a model in which a single type of investors populate the financial market and learn about the expected growth rate of dividends and, separately, about the expected growth rate of output. In that model, as in ours, the expected growth rate of dividends is unobservable and needs to be filtered out, which then contributes positively to the volatility of the stock price. They find that they can match several moments of stock returns. However, their model is not really "closed" since aggregate consumption is not set equal to aggregate dividends plus endowments.

A second class of models studying excess volatility focuses on the discount rate. The literature on the equity-premium puzzle has developed a number of models, such as habit formation models (see Constantinides (1990); Abel (1990); Campbell and Cochrane (1999)), in which the effective discount rate is strongly time varying even though the consumption stream remains very smooth. Using models of that kind, Menzly, Santos, and Veronesi (2004) have recently calibrated a model of the U.S. stock market in which the volatility of stock returns is larger than the one observed in the data.

In a third line of investigation, Bansal and Yaron (2004) and Hansen, Heaton, and Li (2005) find that allowing for a small long-run predictable component in dividend growth rates can generate several observed asset-pricing phenomenon, including volatility of the market return.

Fifthly and finally, our model is related to the historic debate on the stabilizing or destabilizing effects of speculation. Alchian (1950) and Friedman (1953) are given credit for articulating the doctrine according to which agents who do not predict as accurately as others are driven out of the market. De Long, Shleifer, Summers, and Waldmann (1990a, 1991) have indicated that the doctrine may not be correct, but their approach has recently been criticized by Loewenstein and Willard (2005). Sandroni (2000), under the assumption that all investors have equal utility discount rates, shows that agents whose beliefs are most accurate in the entropy sense are the only ones who survive (in terms of wealth accumulation) with probability one in the long run in a complete financial market. As mentioned in the introduction, we also find that eventually rational traders drive out the irrational ones, but rather than happening instantly, this takes a very long time.

\section{Modeling choices and information structure}

We consider a setting similar to that in Scheinkman and Xiong (2003) except that investors are allowed to sell short and, rather than being risk neutral, are risk averse with constant relative risk aversion. The risk aversion does not prevent investors from short selling but it induces them not to take infinitely large 
positions. Moreover, in our model only one group of agents (Group $A$ ) is overconfident while the other category of investors (Group $B$ ) is fully rational.

We now describe the key features of our model. We adopt notation that is similar to the one used in the paper by Scheinkman and Xiong (2003).

\subsection{Process for aggregate output}

The dividend (output) paid by the aggregate economy at time $t$ is equal to $\delta_{t} d t$. The stochastic process for $\delta$ is:

$$
\frac{d \delta_{t}}{\delta_{t}}=f_{t} d t+\sigma_{\delta} d Z_{t}^{\delta}
$$

where $Z^{\delta}$ is a Wiener under the objective probability measure, which governs empirical realizations of the process. The conditional expected growth rate $f_{t}$ of dividend is also stochastic:

$$
d f_{t}=-\zeta\left(f_{t}-\bar{f}\right) d t+\sigma_{f} d Z_{t}^{f} ; \quad \zeta>0,
$$

where $Z^{f}$ is also a Wiener under the objective probability measure.

\subsection{Information structure and filtering}

The conditional expected growth rate of dividends $f$ is not observed by any agent. All investors must estimate, or filter out, the current value of $f$ and its future behavior. They do that from the observation of the current dividend and the observation of a public signal $s$, which has the following process:

$$
d s_{t}=f_{t} d t+\sigma_{s} d Z_{t}^{s}
$$

where $Z^{s}$ is a third Wiener under the objective probability measure as well. All three Wieners, $\left\{Z^{\delta}, Z^{f}, Z^{s}\right\}$, are uncorrelated with each other (under the objective probability measure and any measure equivalent to it) so that, instantaneously, innovations $d Z^{s}$ in the signal convey no information about innovations $d Z^{f}$ in the unobserved variable. Everyone, however, knows that the drift of $s$ at time $t$ is equal to the drift $f_{t}$ of the dividend process. So the signal, as well as the dividend itself, provide some long-run information about the drift of the dividend process. That is the only true reason for which the signal is informative.

There are two groups of agents. Agents in Group $A$ perform their filtering under the delusion that the signal $s$ has strictly positive correlation $\phi \in] 0,1\left[\right.$ with $f$ when, in fact, it is has zero correlation. ${ }^{9}$ The "model" they have in mind is:

$$
d s_{t}=f_{t} d t+\sigma_{s} \phi d Z_{t}^{f}+\sigma_{s} \sqrt{1-\phi^{2}} d Z_{t}^{s} .
$$

Group $B$, on the other hand, is rational (and so knows or learns that $\phi=0$ ). Now, there are two informative roles played by the signal $s$ : from the point of view of all people, the signal provides some information about the drift of the dividend process. But because of the assumed nonzero correlation $\phi$ in the eyes of irrational people, it also provides them with short-run, albeit incorrect, information about the current shock to the dividend growth rate. This single quantity $\phi$ parameterizes the degree of irrationality in the model.

From filtering theory (see Lipster and Shiryaev (2001, Theorem 12.7, page 36)), the conditional expected values, $\widehat{f}^{A}$ and $\widehat{f}^{B}$, of $f$ according to individuals of Group $A$ (deluded; $\phi \neq 0$ ) and Group $B$

\footnotetext{
${ }^{9}$ This is not just a prior, or it is an infinitely precise prior. They refuse to learn the true correlation.
} 
(rational; $\phi=0$ ) obey the following stochastic differential equation: ${ }^{10}$

$$
\begin{aligned}
& d \widehat{f}_{t}^{A}=-\zeta\left(\widehat{f}_{t}^{A}-\bar{f}\right) d t+\frac{\gamma^{A}}{\sigma_{\delta}^{2}}\left(\frac{d \delta}{\delta}-\widehat{f}_{t}^{A} d t\right)+\frac{\phi \sigma_{s} \sigma_{f}+\gamma^{A}}{\sigma_{s}^{2}}\left(d s-\widehat{f}_{t}^{A} d t\right), \\
& d \widehat{f}_{t}^{B}=-\zeta\left(\widehat{f}_{t}^{B}-\bar{f}\right) d t+\frac{\gamma^{B}}{\sigma_{\delta}^{2}}\left(\frac{d \delta}{\delta}-\widehat{f}_{t}^{B} d t\right)+\frac{\gamma^{B}}{\sigma_{s}^{2}}\left(d s-\widehat{f}_{t}^{B} d t\right) .
\end{aligned}
$$

The number $\gamma^{A}\left(\gamma^{B}\right)$ is the steady-state variance of $f$ as estimated by Group $A(B) .{ }^{11}$ These variances would normally be deterministic functions of time. But for simplicity we assume, as did Scheinkman and Xiong (2003), that there has been a sufficiently long period of learning for people of both groups to converge to their level of variance, irrespective of their prior, while, at the same time, agents in Group $A$ have refused to use the same information to infer the correlation number, which is the exact degree to which they are being irrational.

For the purpose of obtaining a martingale, "static" formulation (as done in Cox and Huang (1989) and Karatzas, Lehoczky, and Shreve (1987)), we now rewrite these stochastic differential equations in terms of processes that are Brownian motions under subjective probability measures. Consider a twodimensional process $W^{B}=\left(W_{\delta}^{B}, W_{s}^{B}\right)$ that is Brownian under the probability measure that reflects the expectations of the rational Group $B$. By the definition of the growth rate perceived by Group $B, \widehat{f}^{B}$, we can then write:

$$
\begin{aligned}
\frac{d \delta_{t}}{\delta_{t}} & =\widehat{f}_{t}^{B} d t+\sigma_{\delta} d W_{\delta, t}^{B} \\
d s_{t} & =\widehat{f}_{t}^{B} d t+\sigma_{s} d W_{s, t}^{B}
\end{aligned}
$$

A similar two-dimensional process $W^{A}=\left(W_{\delta}^{A}, W_{s}^{A}\right)$ that is Brownian under $A$ 's probability measure could be defined to represent $B$ 's expectations. The relation between them is:

$$
\begin{aligned}
& d W_{\delta, t}^{B}=d W_{\delta, t}^{A}-\frac{\widehat{f}_{t}^{B}-\widehat{f}_{t}^{A}}{\sigma_{\delta}} d t, \\
& d W_{s, t}^{B}=d W_{s, t}^{A}-\frac{\widehat{f}_{t}^{B}-\widehat{f}_{t}^{A}}{\sigma_{s}} d t .
\end{aligned}
$$

In our model, no agent knows the true state of the economy. Hence, the objective measure is not defined on either agent's $\sigma$-algebra and we can ignore it for the purpose of calculating the equilibrium. We use B's probability measure as the reference measure. From Equations (9) and (10) and Girsanov's theorem, we can determine that the change from $B$ 's measure to $A$ 's measure is given by the exponential martingale:

$$
\eta_{t}=\exp \left(-\frac{1}{2} \int_{0}^{t}\|\nu\|^{2} d t-\int_{0}^{t} \nu_{t}^{\top} d W_{t}^{B}\right)
$$

or

$$
\frac{d \eta_{t}}{\eta_{t}}=-\nu_{t}^{\top} d W_{t}^{B}
$$

\footnotetext{
${ }^{10}$ Observe once again that output $\delta$ serves as a signal, which causes an update of the growth rate of output, just as the signal $s$ does.

${ }^{11}$ The steady-state variances of $f$ as estimated by Group $A$ and Group $B$ are, respectively:$$
\gamma^{A} \triangleq \frac{\sqrt{\left(\zeta+\frac{\phi \sigma_{f}}{\sigma_{s}}\right)^{2}+\left(1-\phi^{2}\right) \sigma_{f}^{2}\left(\frac{1}{\sigma_{s}^{2}}+\frac{1}{\sigma_{\delta}^{2}}\right)}-\left(\zeta+\frac{\phi \sigma_{f}}{\sigma_{s}}\right)}{\frac{1}{\sigma_{s}^{2}}+\frac{1}{\sigma_{\delta}^{2}}}, \quad \gamma^{B} \triangleq \frac{\sqrt{\zeta^{2}+\sigma_{f}^{2}\left(\frac{1}{\sigma_{s}^{2}}+\frac{1}{\sigma_{\delta}^{2}}\right)}-\zeta}{\frac{1}{\sigma_{s}^{2}}+\frac{1}{\sigma_{\delta}^{2}}} .
$$

As has been pointed out by Scheinkman and Xiong (2003), $\gamma^{A}$ decreases as $\phi$ rises, which is the reason for which Group $A$ is called "overconfident". $\gamma^{A}$ starts at the value $\gamma^{B}$ when $\phi=0$ and would reach $\gamma^{A}=0$ when $\phi \rightarrow 1$. The signal can lead Group $A$ ultimately to complete (and foolish) unconditional certainty.
} 
where, defining the difference of opinion by $\widehat{g} \triangleq \widehat{f}^{B}-\widehat{f}^{A}$, we have

$$
\nu_{t}=\left(\widehat{f}_{t}^{B}-\widehat{f}_{t}^{A}\right)\left[\begin{array}{c}
\frac{1}{\sigma_{\delta}} \\
\frac{1}{\sigma_{s}}
\end{array}\right]=\widehat{g}_{t}\left[\begin{array}{c}
\frac{1}{\sigma_{\delta}} \\
\frac{1}{\sigma_{s}}
\end{array}\right] .
$$

The role of $\eta$ is to show how Group $A$ agents over- or under- estimate the probability of a state relative to Group $B$ agents. Girsanov's theorem tells us how current disagreement, $\widehat{f}^{B}-\widehat{f}^{A}$, gets encoded into $\eta$. When, for instance, Group $B$ is currently comparatively pessimistic $\left(\widehat{f}^{B}-\widehat{f}^{A}<0\right)$, Group $A$ views positive innovations in $\delta$ as more probable than Group $B$ does, which is coded by Girsanov as positive innovations in the change of measure $\eta$ for those states of nature in which $\delta$ has positive innovations.

Substituting (7) and (8) into (5) and (6) gives:

$$
\begin{aligned}
d \widehat{f}_{t}^{A}= & {\left[-\zeta\left(\widehat{f}^{A}-\bar{f}\right)+\left(\frac{\gamma^{A}}{\sigma_{\delta}^{2}}+\frac{\phi \sigma_{s} \sigma_{f}+\gamma^{A}}{\sigma_{s}^{2}}\right)\left(\widehat{f}_{t}^{B}-\widehat{f}^{A}\right)\right] d t } \\
& +\frac{\gamma^{A}}{\sigma_{\delta}^{2}} \sigma_{\delta} d W_{\delta, t}^{B}+\frac{\phi \sigma_{s} \sigma_{f}+\gamma^{A}}{\sigma_{s}^{2}} \sigma_{s} d W_{s, t}^{B}, \\
d \widehat{f}_{t}^{B}= & -\zeta\left(\widehat{f}^{B}-\bar{f}\right) d t+\frac{\gamma^{B}}{\sigma_{\delta}} d W_{\delta, t}^{B}+\frac{\gamma^{B}}{\sigma_{s}} d W_{s, t}^{B} .
\end{aligned}
$$

The conditional variance of $\widehat{f}^{A}$ is equal to:

$$
\left[\frac{\gamma^{A}}{\sigma_{\delta}}\right]^{2}+\left[\phi \sigma_{f}+\frac{\gamma^{A}}{\sigma_{s}}\right]^{2}=-2 \zeta \gamma^{A}+\sigma_{f}^{2}
$$

which is a monotonically increasing function of $\phi$, rising from $-2 \zeta \gamma^{B}+\sigma_{f}^{2}$ at $\phi=0$ to $\sigma_{f}^{2}$ at $\phi=1$. That is, the irrational investor Group $A$ changes its beliefs in a more volatile way than does Group $B$. It is also important to notice that the coefficient of $\left(\widehat{f}_{t}^{B}-\widehat{f}_{t}^{A}\right)$ in Equation (14), $\left(\frac{\gamma^{A}}{\sigma_{\delta}^{2}}+\frac{\phi \sigma_{s} \sigma_{f}+\gamma^{A}}{\sigma_{s}^{2}}\right)$, is positive. This means that, in the eyes of the rational investors, the beliefs of the irrational ones are expected to revert towards the beliefs of the rational investors (in addition to both reverting to the long-run average growth rate).

For later reference, we also write the process for the difference of opinion, $\widehat{g}$ :

$$
d \widehat{g}_{t}=-\left(\zeta+\frac{\gamma^{A}}{\sigma_{\delta}^{2}}+\frac{\phi \sigma_{s} \sigma_{f}+\gamma^{A}}{\sigma_{s}^{2}}\right) \widehat{g}_{t} d t+\frac{\gamma^{B}-\gamma^{A}}{\sigma_{\delta}} d W_{\delta, t}^{B}+\frac{\gamma^{B}-\left(\phi \sigma_{s} \sigma_{f}+\gamma^{A}\right)}{\sigma_{s}} d W_{s, t}^{B} .
$$

When $\widehat{g}=\widehat{f}^{B}-\widehat{f}^{A}>0$, Group $B$ investors are comparatively optimistic or Group $A$ comparatively pessimistic. Also, $\widehat{g}$ (or its absolute value) can be viewed as a measure of the dispersion of beliefs or opinions. Because $\gamma^{B}-\left(\phi \sigma_{s} \sigma_{f}+\gamma^{A}\right) \leq 0$, a positive realization of the signal increment $d W_{s, t}^{B}$ causes Group $A$ to become more optimistic relative to what it was before. As noted, the rational group expects the difference in beliefs to revert to zero.

\subsection{Properties of the state process}

The Markovian system made of (7), (8), (14) and (15) completely characterizes the evolution of the exogenous part of the economy in the eyes of Group $B$. The joint dynamics of the four state variables, $\left\{\delta, \eta, \widehat{f}^{B}, \widehat{g}\right\}$, are provided by Equations (7), (12), (15) and (17). They are driven by only two Brownians, $W_{\delta}^{B}$ and $W_{s}^{B}$, because variable $f$ is unobserved by anyone and is only a latent variable. Therefore, the four variables are by no means independent of each other. Since there are only two Brownians, the diffusion matrix of $\left\{\delta, \eta, \widehat{f}^{B}, \widehat{g}\right\}$ is a $4 \times 2$ matrix:

$$
\left[\begin{array}{cc}
\delta \sigma_{\delta}>0 & 0 \\
-\eta \frac{\widehat{g}}{\sigma_{\delta}} & -\eta \frac{\widehat{g}}{\sigma_{s}} \\
\frac{\gamma^{B}}{\sigma_{\delta}}>0 & \frac{\gamma^{B}}{\sigma_{s}}>0 \\
\frac{\gamma^{B}-\gamma^{A}}{\sigma_{\delta}} \geq 0 & \frac{\gamma^{B}-\left(\phi \sigma_{s} \sigma_{f}+\gamma^{A}\right)}{\sigma_{s}} \leq 0
\end{array}\right] .
$$


Evidently, $\delta$ and $\widehat{f}^{B}$ are always positively correlated with each other. The diffusion vector of $\eta$ has the sign opposite to the sign of $\widehat{g}$. The covariance of variables $\delta$ and $\eta$ will play a central role in what follows. Instantaneously, it is equal to $-\delta \eta \widehat{g}$. Thus, $\delta$ and $\eta$ covary positively when $\widehat{g}<0$ and negatively in the opposite case.

In the special case of pure Bayesian learning, in which everyone is rational $(\phi=0)$ and differences in beliefs can arise only from differences in priors, $\gamma^{A}=\gamma^{B}$ so that $\widehat{g}$ has zero diffusion and reverts to zero deterministically (see Equation (17)). However, even in that case, as long as $\widehat{g}$ has not reached the value $0, \eta$ fluctuates randomly as public signals $(\delta, s)$ are realized.

Of the four state variables, two have a direct, immediate effect on the economy. They are $\delta$ and $\eta$. We propose to call $\delta$ "the fundamental" and $\eta$ "sentiment". The "fundamental" moves on its own but "sentiment" is affected by the fundamental because realizations of the dividend provide information. ${ }^{12}$ The other two state variables, $\widehat{f}^{B}$ and $\widehat{g}$, have only an indirect effect in that they only act on the first two: $\widehat{f}^{B}$ is the current estimate of the drift of $\delta$, and $\widehat{g}$ determines the diffusion of $\eta$. The state variable $\widehat{g}$ will be called "disagreement" and, later, "dispersion of beliefs". We emphasize that two state variables - $\widehat{g}$ and $\eta$ - are needed to capture the dynamics of heterogeneous belief and their effects, unlike what happens in, e.g., Barberis, Shleifer, and Vishny (1998).

In the proposition below, we list the effects of irrationality and heterogeneity of beliefs.

Proposition 1 There are two distinct effects of imperfect learning and heterogeneous beliefs:

- Effect \#1 (effect of imperfect learning): $\widehat{g}$ has nonzero diffusion; disagreement is stochastic. Even if the two groups of investors happened to agree today about the growth rate of aggregate dividends $(\widehat{g}=0)$, all investors would still know that they will revise their future estimates of the growth rate. In particular, the rational investors are conscious of the fact that irrational investors will revise their estimate in a manner that differs from theirs, so that they know that they will not agree tomorrow. This effect is instantaneous.

- Effect \#2 (effect of heterogeneous beliefs): $\widehat{g}$ affects the diffusion of $\eta$; disagreement is sentiment risk. One group of investors may not agree today with the other one about its estimate of the current rate of growth of dividend, that is, $\widehat{g} \neq 0$. This effect is cumulative: $\widehat{g}$, which is stochastic as per Effect \#1, scales the diffusion of $\eta$, which implies that $\eta$ has a diffusion that can take large positive or negative values.

The joint conditional distribution of $\eta_{u}$ and $\delta_{u}$, given $\delta_{t}, \eta_{t}, \widehat{f}_{t}^{B}, \widehat{g}_{t}$ at $t$ will be needed to characterize prices and portfolio policies. That joint distribution is not easy to obtain but its characteristic or moment generating function $\mathbb{E}_{\widehat{f}^{B}, \widehat{g}}^{B}\left[\left(\frac{\delta_{u}}{\delta}\right)^{\varepsilon}\left(\frac{\eta_{u}}{\eta}\right)^{\chi}\right] ; u \geq t ; \varepsilon, \chi \in \mathbb{R}$ (or $\mathbb{C}$ ) can be obtained in closed form. We decompose the moment generating function into a product of two terms and give names to the corresponding functions:

$$
\mathbb{E}_{\widehat{f}^{B}, \widehat{g}}^{B}\left[\left(\frac{\delta_{u}}{\delta}\right)^{\varepsilon}\left(\frac{\eta_{u}}{\eta}\right)^{\chi}\right]=H_{f}\left(\widehat{f}^{B}, t ; u, \varepsilon\right) \times H_{g}(\widehat{g}, t, u ; \varepsilon, \chi),
$$

where:

$$
\begin{aligned}
H_{f}\left(\widehat{f}^{B}, t ; u, \varepsilon\right) & \triangleq \mathbb{E}_{\widehat{f}^{B}}^{B}\left[\left(\frac{\delta_{u}}{\delta}\right)^{\varepsilon}\right], \\
H_{g}(\widehat{g}, t, u ; \varepsilon, \chi) & \triangleq \frac{\mathbb{E}_{\widehat{f}^{B}, \widehat{g}}^{B}\left[\left(\frac{\delta_{u}}{\delta}\right)^{\varepsilon}\left(\frac{\eta_{u}}{\eta}\right)^{\chi}\right]}{\mathbb{E}_{\widehat{f}^{B}}^{B}\left[\left(\frac{\delta_{u}}{\delta}\right)^{\varepsilon}\right]} .
\end{aligned}
$$

Guided by the functional form of the coefficients of the associated partial differential equations, we present fully explicit formulae for $H_{f}$ and $H_{g}$ in Lemma 1 of Appendix A. ${ }^{13}$

\footnotetext{
${ }^{12}$ It will soon become apparent that, in equilibrium, changes in $\eta$ will also be changes in the relative weights, or consumption shares, of the two subpopulations.

${ }^{13}$ These formulae are generalization of Heston (1993) and Kim and Omberg (1996) and perhaps others.
} 
Further decomposing the moment generating function as

$$
\begin{aligned}
& \mathbb{E}_{\widehat{f}^{B}, \widehat{g}}^{B}\left[\left(\frac{\delta_{u}}{\delta}\right)^{\varepsilon}\left(\frac{\eta_{u}}{\eta}\right)^{\chi}\right] \\
& =\mathbb{E}_{\widehat{f}^{B}}^{B}\left[\left(\frac{\delta_{u}}{\delta}\right)^{\varepsilon}\right] \times\left\{\mathbb{E}_{\widehat{g}}^{B}\left[\left(\frac{\eta_{u}}{\eta}\right)^{\chi}\right]+\left[\frac{\mathbb{E}_{\widehat{f}^{B}, \widehat{g}}^{B}\left[\left(\frac{\delta_{u}}{\delta}\right)^{\varepsilon}\left(\frac{\eta_{u}}{\eta}\right)^{\chi}\right]}{\mathbb{E}_{\widehat{f}^{B}}^{B}\left[\left(\frac{\delta_{u}}{\delta}\right)^{\varepsilon}\right]}-\mathbb{E}_{\widehat{g}}^{B}\left[\left(\frac{\eta_{u}}{\eta}\right)^{\chi}\right]\right]\right\} \\
& =H_{f}\left(\widehat{f}^{B}, t ; u, \varepsilon\right) \times\left\{H_{g}(\widehat{g}, t, u ; 0, \chi)+\left[H_{g}(\widehat{g}, t, u ; \varepsilon, \chi)-H_{g}(\widehat{g}, t, u ; 0, \chi)\right]\right\},
\end{aligned}
$$

leads to the following:

Definition 1 We call the first term of this product (22) "the effect of the growth and variance of $\delta$ ". The second term (within braces) of the product is the joint effect of the variance of $\eta$ and the correlation between $\delta$ and $\eta$. It is broken into two additive pieces. We call the first piece "the effect of the variance of $\eta "(\varepsilon=0)$ and the second piece "the effect of the correlation between $\delta$ and $\eta$ ".

We also establish in Appendix A a number of properties of the moment generating function that will be useful in understanding the behavior of equilibrium prices and portfolios. These are stated in the lemma below.

Lemma 2 The moment generating function (19) has the following properties for $0 \leq \chi \leq 1:^{14}$

1. The effect of the variance of sentiment, $\eta$, is to weakly reduce $H_{g}$ (i.e., $\left.\mathbb{E}_{\widehat{g}}^{B}\left[\left(\frac{\eta_{u}}{\eta}\right)^{\chi}\right] \leq 1\right)$. In the absence of the correlation effect (i.e., when $\varepsilon=0$ ), the function $H_{g}$ is symmetric and, in the neighborhood of $\widehat{g}=0$, is concave with respect to $\widehat{g}$.

2. When $\varepsilon \times \widehat{g} \geq 0$ (i.e., $\varepsilon \leq 0$ and $\widehat{g} \leq 0$, or $\varepsilon \geq 0$ and $\widehat{g} \geq 0$ ), the effect of the correlation between the fundamental, $\delta$, and sentiment, $\eta$, is nonpositive for any maturity (i.e., $H_{g}(\widehat{g}, t, u ; \varepsilon, \chi)-$ $\left.H_{g}(\widehat{g}, t, u ; 0, \chi) \leq 0\right)$.

3. For $\widehat{g}=0, H_{g}=1$ if $\phi=0$. When $u>t$, then for $\widehat{g}=0, H_{g}$ is strictly smaller than 1 if $\phi>0$.

4. The logarithmic derivative $\frac{1}{H_{g}} \frac{\partial H_{g}}{\partial \widehat{g}}$ is a straight-line, nonincreasing function of $\widehat{g}$. In a neighborhood of $\widehat{g}=0$, with $\varepsilon<0$, the derivative $\frac{1}{H_{g}} \frac{\partial H_{g}}{\partial \widehat{g}}$ is nonnegative.

5. The logarithmic derivative $\frac{1}{H_{f}} \frac{\partial H_{f}}{\partial \widehat{f}^{B}}$ has the sign of $\varepsilon$.

Statement 1 of the lemma follows directly from Jensen's inequality. An obvious consequence of it is that, for any random variable $X_{u}$ that is uncorrelated with $\eta_{u}$, we have: $\mathbb{E}_{\delta, \eta, \widehat{f}^{B}, \widehat{g}}\left[\left(\frac{\eta_{u}}{\eta}\right)^{\chi} X_{u}\right] \leq$ $\mathbb{E}_{\delta, \eta, \widehat{f}^{B}, \widehat{g}}^{B}\left[X_{u}\right]$. Statement 1 also says that only $\widehat{g}^{2}$ not the sign of $\widehat{g}$ matters for the effect of the variance of $\eta$. An increase in $\widehat{g}$ intensifies the (negative) effect of the variance of sentiment on $H_{g}$. This is because $\widehat{g}^{2}$ scales the variance of $\eta$. The first panel of Figure 1 illustrates the effect of the variance of $\eta$ as a function of $\widehat{g}$.

Statement 2 says that the effect of the correlation between the fundamental $\delta$ and sentiment is negative if $\varepsilon \times \widehat{g} \geq 0$. If $\varepsilon \times \widehat{g}<0$, the effect of the correlation is ambiguous. The second panel of Figure 1 shows the variance and correlation effects described above. When $\phi=0, H_{g}=1$; this is the horizontal, dotted line. The difference between this curve and the other curves in this plot shows the effect of irrationality on $H_{g}$. The solid curve, which is for $\varepsilon=0$, reflects only the variance effect of

\footnotetext{
${ }^{14}$ The reason we consider only values of $0 \leq \chi \leq 1$ is that the expressions for prices that we are going to derive will contain only these powers of $\eta$.
} 
Figure 1: Properties of the moment generating function
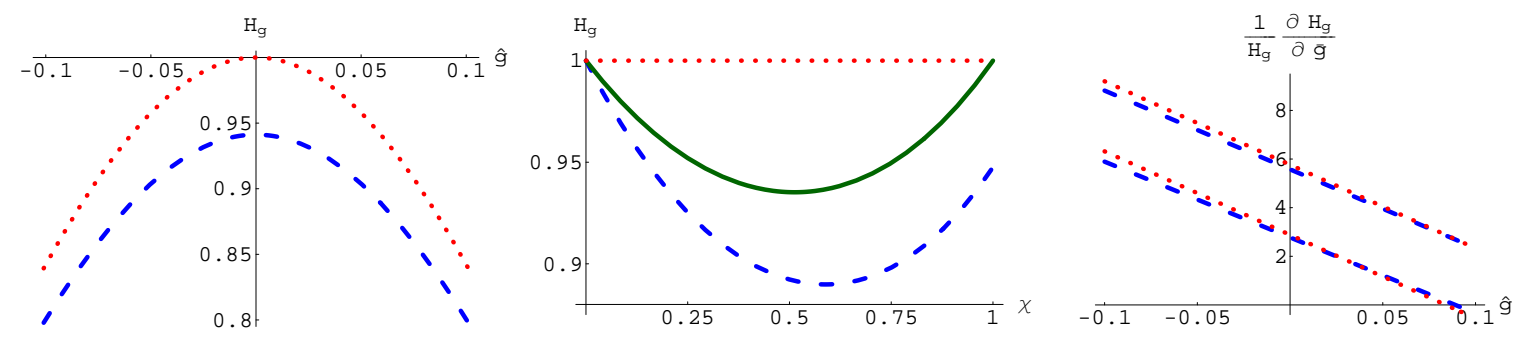

The first panel of the figure plots $H_{g}$ against $\widehat{g}$ for different $\phi$, with $\varepsilon=0$ and $\chi=2 / 3$. The dotted curve is for the case of $\phi=0$ and the dashed curve is for the case of $\phi=0.95$. The second panel plots $H_{g}$ against $\chi$ for different $\phi$ and $\varepsilon$, with $\widehat{g}=0$. The dotted horizontal line is $H_{g}$ for the case of $\phi=0$. The two other curves are for $\phi=0.95$. The solid curve, which is for $\varepsilon=0$, reflects only the variance effect of sentiment; the dashed curve, which is for $\varepsilon=-2$, reflects both the variance and correlation effects. The third panel plots $\frac{1}{H_{g}} \frac{\partial H_{g}}{\partial \widehat{g}}$ against $\widehat{g}$, with $\varepsilon=-2$ and $\chi=1 / 3$ (lower pair of lines) and $\chi=2 / 3$ (upper pair of lines). Other parameter values used here are given in Table 1.

sentiment; the dashed curve, which is for $\varepsilon=-2$, reflects both the variance and correlation effects. Thus, the difference between the dashed lower curve and the solid higher curve reflects the effect of correlation between $\delta$ and $\eta$.

Statement 3 shows that the effect of irrationality (that is, a positive $\phi$ ) is to reduce $H_{g}$ when $\widehat{g}=0$. If $\widehat{g}>0$, however, the variations of $H_{g}$ with respect to $\phi$ are generally ambiguous; if the irrational investors are optimistic, their optimism counteracts their irrationality. The effect of $\phi$ on $H_{g}$ can be seen in the first panel of Figure 1 by comparing the intercepts of the two curves for $H_{g}$ : the dotted one for $\phi=0$ and the dashed one for $\phi=0.95$ (both for the case of $\varepsilon=0$ ). The effect of imperfect learning can also be seen in the second panel of Figure 1 by comparing the dotted line with the dashed curve.

The properties described in Statement 4 are illustrated in the third panel of Figure 1, which plots $\frac{1}{H_{g}} \frac{\partial H_{g}}{\partial \widehat{g}}$ against $\widehat{g}$, for two values of $\phi$ and two values of $\chi$. Note that a change in $\phi$ does not modify the derivative appreciably.

\section{Individual optimization and equilibrium}

In this section, we first describe the optimization problem faced by each investor and then, assuming complete financial markets, the equilibrium in the economy. This includes a characterization of the instantaneously riskless interest rate and the market price of risk and also the pricing of single-maturity claims. We conclude this section by explaining how the complete-markets equilibrium can be implemented via dynamic trading in long-lived securities and how one can determine the prices of these securities.

\subsection{Preferences of agents and their optimization problems}

We are interested in the interaction between two groups, one of which is rational and the other is not. Differences in risk aversion and differences in the rate of impatience are not our main focus. So, we restrict our analysis to the setting in which both groups have power utility with the same risk aversion, $1-\alpha$, and rate of impatience, $\rho$. 
Assuming a complete financial market, ${ }^{15}$ the problem of Group $B$ is to maximize the expected utility from lifetime consumption:

$$
\sup _{c} \mathbb{E}^{B} \int_{0}^{\infty} e^{-\rho t} \frac{1}{\alpha}\left(c_{t}^{B}\right)^{\alpha} d t ; \quad \alpha<1,
$$

subject to the static budget constraint:

$$
\mathbb{E}^{B} \int_{0}^{\infty} \xi_{t}^{B} c_{t}^{B} d t=\bar{\theta}^{B} \mathbb{E}^{B} \int_{0}^{\infty} \xi_{t}^{B} \delta_{t} d t
$$

where $\xi^{B}$ is the change of measure from Group $B$ 's probability measure to the risk neutralized measure (which we determine in the next section) and $\bar{\theta}^{B}$ is the share of equity with which $B$ is initially endowed. The first-order condition for consumption equates marginal utility to $\lambda^{B} \xi_{t}^{B}$, where $\lambda^{B}$ is the Lagrange multiplier of the budget constraint (24):

$$
e^{-\rho t}\left(c_{t}^{B}\right)^{\alpha-1}=\lambda^{B} \xi_{t}^{B}
$$

Group $A$ is assumed to have the same utility function and an initial share $\bar{\theta}^{A}=1-\bar{\theta}^{B}$ of the equity, and thus, an analogous optimization problem. The only difference is that Group $A$ uses a probability measure that is different from that of Group $B$. The problem of Group $A$ is to maximize the expected utility from lifetime consumption:

$$
\sup _{c} \mathbb{E}^{A} \int_{0}^{\infty} e^{-\rho t} \frac{1}{\alpha}\left(c_{t}^{A}\right)^{\alpha} d t
$$

subject to the static budget constraint:

$$
\mathbb{E}^{A} \int_{0}^{\infty} \xi_{t}^{A} c_{t}^{A} d t=\bar{\theta}^{A} \mathbb{E}^{A} \int_{0}^{\infty} \xi_{t}^{A} \delta_{t} d t
$$

where $\xi^{A}$ is the change of measure from agents $A$ 's probability measure to the risk neutralized measure. ${ }^{16}$ The problem of $A$ can be restated under $B$ 's probability measure as follows:

$$
\sup _{c} \mathbb{E}^{B} \int_{0}^{\infty} \eta_{t} e^{-\rho t} \frac{1}{\alpha}\left(c_{t}^{A}\right)^{\alpha} d t
$$

subject to the static budget constraint:

$$
\mathbb{E}^{B} \int_{0}^{\infty} \xi_{t}^{B} c_{t}^{A} d t=\bar{\theta}^{A} \mathbb{E}^{B} \int_{0}^{\infty} \xi_{t}^{B} \delta_{t} d t
$$

The first-order condition for consumption in this case is

$$
\eta_{t} e^{-\rho t}\left(c_{t}^{A}\right)^{\alpha-1}=\lambda^{A} \xi_{t}^{B},
$$

where $\lambda^{A}$ is the Lagrange multiplier of the budget constraint (29).

\footnotetext{
${ }^{15}$ David (2004) says that the fluctuating difference of measure $\eta$ between the two groups makes the market "effectively incomplete". That is a matter of semantics. Analytically, the equilibrium can be obtained by complete-market methods. It would probably be more descriptive of the analytical structure that is reflected in Equation (28) below, to say that the fluctuating $\eta$ causes the utility function of agents $A$ to become "effectively state dependent" (i.e. non von NeumanMorgenstern) relative to the probability measure of Group $B$.

${ }^{16} \xi^{A}$ is the density that makes prices martingales under $A^{A}$ 's probability measure. $\xi^{B}$ is the density that makes prices martingales under $B$ 's probability measure. For any event $E: \mathbb{E}^{A}\left[\xi^{A} \mathbf{1}_{E}\right]=\mathbb{E}^{B}\left[\eta \xi^{A} \mathbf{1}_{E}\right]=\mathbb{E}^{B}\left[\xi^{B} \mathbf{1}_{E}\right]$, which implies that $\xi^{B}=\eta \xi^{A}$. The martingale pricing density is defined relative to each agent's probability measure. But the risk neutral measure is the same in the end.
} 


\subsection{Equilibrium pricing measure}

An equilibrium is a price system and a pair of consumption-portfolio processes such that: (i) investors choose their optimal consumption-portfolio strategies, given their perceived price processes; (ii) the perceived security price processes are consistent across investors; and (iii) commodity and securities markets clear.

The aggregate resource constraint, from (25) and (30), is

$$
\left(\frac{\lambda^{A} \xi_{t}^{B} e^{\rho t}}{\eta_{t}}\right)^{\frac{1}{\alpha-1}}+\left(\lambda^{B} \xi_{t}^{B} e^{\rho t}\right)^{\frac{1}{\alpha-1}}=\delta_{t} .
$$

Solving this equation:

$$
\xi_{t}^{B}\left(\delta_{t}, \eta_{t}\right)=e^{-\rho t}\left[\left(\frac{\eta_{t}}{\lambda^{A}}\right)^{\frac{1}{1-\alpha}}+\left(\frac{1}{\lambda^{B}}\right)^{\frac{1}{1-\alpha}}\right]^{1-\alpha} \delta_{t}^{\alpha-1},
$$

and, therefore:

$$
\begin{aligned}
c_{t}^{A} & =\delta_{t} \omega\left(\eta_{t}\right), \\
c_{t}^{B} & =\delta_{t}\left(1-\omega\left(\eta_{t}\right)\right),
\end{aligned}
$$

where: ${ }^{17}$

$$
\omega\left(\eta_{t}\right) \triangleq \frac{\left(\frac{\eta_{t}}{\lambda^{A}}\right)^{\frac{1}{1-\alpha}}}{\left(\frac{\eta_{t}}{\lambda^{A}}\right)^{\frac{1}{1-\alpha}}+\left(\frac{1}{\lambda^{B}}\right)^{\frac{1}{1-\alpha}}}
$$

is the share of consumption of Group $A .{ }^{18}$ The consumption-sharing rule is linear in $\delta$ because both groups have the same risk aversion. But the slope of the linear relation, that is, the share of consumption allocated to each group $\omega(\eta)$, is stochastic and driven by sentiment, $\eta$, because of the improper use of signal by individuals of Group $A$.

The equilibrium value of $\xi^{B}$ - the martingale pricing density under $B^{\prime}$ s probability measure depends on $\eta$, the probability density of $A$ relative to $B$, that is, sentiment. In addition to reflecting the abundance or scarcity of goods, as is usual in the absence of state preference or heterogeneous beliefs, the state prices also incorporate a power (or Hölder) average of the probability beliefs of the two groups (given by the term that is in square brackets in Equation (32)). As $\eta$ fluctuates, average probability belief or "sentiment" fluctuates with it. In writing his/her budget constraint based on $\xi^{B}$, Agent $B$ anticipates $A$ 's beliefs. This reflects "higher-order beliefs."

Given the constant multipliers $\lambda^{A}$ and $\lambda^{B}$, and given the exogenous process for the fundamental, $\delta$, and sentiment, $\eta$, we have now characterized the complete-market equilibrium. It would only remain to relate the Lagrange multipliers $\lambda^{A}$ and $\lambda^{B}$ to the initial endowments. This requires the calculation of the wealth of each group, which is done in Equation (47) below. Equilibrium prices will be built from future values of $\xi^{B}$, which itself is a function of the future fundamental and sentiment. In the previous section we have already discussed the distributional properties of the fundamental and sentiment. We now discuss the effect of these two state variables on the risk-neutral pricing measure, $\xi^{B}(\delta, \eta)$.

Notice in Equation (32) that the functional forms of $\xi^{B}$ with respect to $\delta$ and with respect to $\eta$ are very different from each other. This is because fundamental risk and sentiment risk have very different economic effects on utility and marginal utility. From Equation (32), one can show that the

\footnotetext{
${ }^{17}$ For arbitrary (but von Neuman Morgenstern) utility, $\omega$ would be defined as the ratio of Group $A$ 's absolute risk tolerance over the sum of absolute risk tolerances of Group A and Group B. See Lintner (1969) and Basak (2004). In the isoelastic case, this ratio reduces to the share of consumption.

${ }^{18}$ Along any sample path of the economy, $\omega(\eta)$ is monotonically increasing with $\eta$. Thus, we can use $\omega$ as a representation of $\eta$. This is the sense in which changes in $\eta$ are equivalent to changes in the relative weights, or consumption shares, of the two subpopulations.
} 
first derivative of $\xi^{B}(\delta, \eta)$ with respect to $\delta$ is negative while the second derivative is positive. The second derivative of the function $\xi^{B}(\delta, \eta)$ with respect to $\eta$ has the same sign as $\alpha$. The cross derivative of the function $\xi^{B}(\delta, \eta)$ is unambiguously negative. These derivatives have the following economic interpretation.

The fundamental, $\delta$, has the customary aggregate effect on both groups: when output increases, marginal utility decreases. Thus, an increase in future expected output decreases the expected value of discount factors. Furthermore, marginal utility is convex with respect to $\delta$. Jensen's inequality implies that an increase in fundamental risk increases the expected value of discount factors, which is the familiar precautionary-saving motive. ${ }^{19}$

In contrast to the fundamental, which is an aggregate shock, sentiment acts like a wedge between the two groups. ${ }^{20}$ Because the second derivative of $\xi^{B}(\delta, \eta)$ with respect to $\eta$ has the same sign as $\alpha$, if $\alpha<0$ (risk aversion greater than 1), discount factors are concave with respect to $\eta$ so that an increase in sentiment risk (the variance of $\eta$ ), by Jensen's inequality, reduces the expected values of all the future stochastic discount factors written with respect to $B$ 's measure. The exact nature of this property will be reflected in the following subsections and Theorem 2 below.

\subsection{Instantaneous pricing of risk and rate of interest}

The rate of interest and the prices of risk in this equilibrium are implied by the pricing measure in (32). Defining, as in Cox and Huang (1989), the rate of interest $r$ on an instantaneous maturity deposit as the drift of the risk-neutralized measure for Group $i$, and the market prices of risk in the eyes of Group $i=\{A, B\}$ denoted by the vector $\kappa^{i}$ as the diffusion of the risk-neutralized measure for Group $i$, where the risk-neutralized measure is:

$$
\xi_{t}^{i}=\delta_{0}^{\alpha-1} \exp \left(-\int_{0}^{t} r d t-\frac{1}{2} \int_{0}^{t}\left\|\kappa^{i}\right\|^{2} d t-\int_{0}^{t}\left(\kappa^{i}\right)^{\top} d W^{i}\right)
$$

one can obtain the interest rate and the market prices of risk by applying Itô's lemma to (32).

Theorem 1 In equilibrium, the instantaneous interest rate is

$$
\begin{aligned}
r\left(\eta, \widehat{f}^{B}, \widehat{g}\right)= & \rho+(1-\alpha) \widehat{f}^{B}-\frac{1}{2}(1-\alpha)(2-\alpha) \sigma_{\delta}^{2}-(1-\alpha) \widehat{g} \omega(\eta) \\
& -\frac{1}{2}\left(\frac{\alpha}{1-\alpha}\right)\left(\frac{1}{\sigma_{\delta}^{2}}+\frac{1}{\sigma_{s}^{2}}\right) \widehat{g}^{2} \omega(\eta)[1-\omega(\eta)],
\end{aligned}
$$

and the market prices of risk in the eyes of Groups $B$ and $A$ are: $:^{21}$

$$
\begin{aligned}
& \kappa^{B}(\eta, \widehat{g})=\left[\begin{array}{c}
(1-\alpha) \sigma_{\delta} \\
0
\end{array}\right]+\widehat{g} \omega(\eta)\left[\begin{array}{c}
\frac{1}{\sigma_{\delta}} \\
\frac{1}{\sigma_{s}}
\end{array}\right], \\
& \kappa^{A}(\eta, \widehat{g})=\left[\begin{array}{c}
(1-\alpha) \sigma_{\delta} \\
0
\end{array}\right]-\widehat{g}[1-\omega(\eta)]\left[\begin{array}{c}
\frac{1}{\sigma_{\delta}} \\
\frac{1}{\sigma_{s}}
\end{array}\right] .
\end{aligned}
$$

\footnotetext{
${ }^{19}$ For arbitrary (but von Neuman Morgenstern) utility, the curvature of $\xi^{B}$ with respect to the fundamental $\delta$ is equal to total absolute risk aversion multiplied by total absolute prudence. See Basak (2004).

${ }^{20}$ For arbitrary (but von Neuman Morgenstern) utility, Basak (2004) shows that the curvature of the $\xi^{B}$ with respect to $\eta$ is given by a combination of the risk aversions and the prudences of the two groups. One can verify on his formula that the knife-edge case of zero curvature is the case in which both groups have log utility. A special case of his result is obtained here for isoelastic utility.

${ }^{21}$ The risk-neutral measures for Groups $A$ and $B$ differ only in the market prices of risk. That is, the instantaneously riskless interest rate perceived by all agents is the same, and so the difference in the risk neutral measures is purely a difference in the market prices of risk perceived by the two groups.
} 
The rate of interest, from equation (37), is an increasing function of Group B's expected rate of growth of the dividend, $\widehat{f}^{B}$. In fact, expectation of future growth is impounded only in the rate of interest and not in the prices of risk.

Observe also that the rate of interest is influenced in a nonmonotonic and asymmetric way by the difference in beliefs, $\widehat{g}$, as it is in David (2004). The asymmetry occurs because $\widehat{g}$ contributes both to the average of $\widehat{f}^{A}$ and $\widehat{f}^{B}$, and also to the difference between them. To highlight this point, one could define an average belief:

$$
\widehat{f}^{M} \triangleq \widehat{f}^{A} \times \omega(\eta)+\widehat{f}^{B} \times(1-\omega(\eta)) .
$$

The rate of interest can then be written:

$$
\begin{aligned}
r\left(\eta, \widehat{f}^{M}, \widehat{g}\right)= & \rho+(1-\alpha) \widehat{f}^{M}-\frac{1}{2}(1-\alpha)(2-\alpha) \sigma_{\delta}^{2} \\
& -\frac{1}{2}\left(\frac{\alpha}{1-\alpha}\right)\left(\frac{1}{\sigma_{\delta}^{2}}+\frac{1}{\sigma_{s}^{2}}\right) \widehat{g}^{2} \omega(\eta)[1-\omega(\eta)] .
\end{aligned}
$$

Holding average belief fixed, the effect of $\widehat{g}$, which appears in the last term is now purely quadratic and symmetric. In this way, $\widehat{g}$ represents the effect of pure dispersion of beliefs in the population. Once $\widehat{f}^{M}$ has been introduced as a variable, the second derivative of $\xi^{B}$ with respect to $\eta$ is the sole cause of the influence of $\widehat{g}$, which drives the variance of $\eta$. The effect of the cross derivative of $\xi^{B}$ with respect to $\eta$ and $\delta$ and of the covariance between these two variables has now been absorbed into $f^{M}{ }^{22}$ The very last terms of Equations (37) and (41), containing $\widehat{g}^{2}$, arise from the second derivative of $\xi^{B}$ with respect to $\eta$, which, as we discussed in Subsection 4.2, is negative whenever risk aversion is greater than $1(\alpha<0)$. When that is true, disagreement increases the equilibrium rate of interest because it depresses all the stochastic discount factors. ${ }^{23}$

We now discuss the prices of risk, $\kappa$, which are the expected excess returns on a unit of exposure to the fundamental and signal shocks, $W_{\delta}$ and $W_{s}$. From (38) and (39), we see that under agreement $(\widehat{g}=0)$, the prices of risk $\kappa$ include a reward for fundamental risk $W_{\delta}$ but zero reward for signal risk $W_{s}$. As soon as there is disagreement, both groups of investors realize that "sentiment", that is, the probability measure of the other group, will fluctuate randomly. Hence, they start charging a premium for the risk arising from the vagaries of others.

It is noteworthy that neither the rate of interest nor the prices of risk depend directly on the parameter $\phi$ measuring irrationality. They depend on $\phi$ indirectly via the current value of the probability difference, $\eta$, and the current value of the difference of opinion, $\widehat{g}$.

\footnotetext{
${ }^{22}$ It is also conceivable to recognize average beliefs, $\widehat{f}^{M}$, and dispersion of beliefs, $\widehat{g}$, as two drivers of not just the price of the riskless instantaneous deposit but also for the prices of other securities. It is unfortunately not possible to define a single concept of "average belief" that would be valid for assets of all maturities. The way in which beliefs compound over time and get discounted into prices via marginal utility, when $\eta$ is stochastic (and generally correlated with $\delta$ ), would imply a different concept of average beliefs for different maturities. The average belief $\widehat{f}^{M}$ that we have defined in (40) applies only to the rate of interest, which is an instantaneous-maturity asset. Keeping $\widehat{f}^{M}$ fixed is not sufficient to keep the average measure $M$ fixed. This point, which also arises in Jouini and Napp (2003), is related to the observation made by Allen, Morris, and Shin (2004) that, under risk aversion, market-average beliefs do not satisfy the law of iterated expectations. Note that $\widehat{f}^{M}$ is the drift of $\delta$ under an average probability belief/measure $M$ defined by the change of measure (from $B$ 's measure to $M$ ):

$$
\frac{\left[\left(\frac{\eta_{t}}{\lambda^{A}}\right)^{\frac{1}{1-\alpha}}+\left(\frac{1}{\lambda^{B}}\right)^{\frac{1}{1-\alpha}}\right]^{1-\alpha}}{\left[\left(\frac{1}{\lambda^{A}}\right)^{\frac{1}{1-\alpha}}+\left(\frac{1}{\lambda^{B}}\right)^{\frac{1}{1-\alpha}}\right]^{1-\alpha}},
$$

and this process does not have zero drift. It generates probability densities that do not sum to 1 and do not satisfy the law of iterated expectations. This issue does not arise in a one-period model such as Gollier (2005).

${ }^{23}$ David (2004) assumed a risk aversion lower than 1, precisely in order to bring down the rate of interest. See our numerical illustrations below. Had we assumed a lifetime utility of the recursive, Epstein-Zin type, we could have distinguished risk aversion from elasticity of intertemporal substitution. It is likely that the condition for the rate of interest to be reduced (increased) by dispersion of beliefs would have hinged on the elasticity of substitution being higher (lower) than 1 , not on the level of risk aversion.
} 


\subsection{Pricing of sentiment risk in single-maturity claims}

The rate of interest and the prices of risk characterize the pricing of securities over the immediate, infinitesimal future period of time. To characterize the pricing of single-maturity claims, we need to compound the discounting from the present time $t$ to some future maturity date $u$. That means we need to establish the properties of the conditional expected values of future discount factors: $\mathbb{E}_{\eta, \widehat{f}^{B}, \widehat{g}}\left[\frac{\xi_{u}^{B}}{\xi_{t}^{B}}\right]$ as functions of $\eta, \widehat{f}^{B}$ and $\widehat{g}$. That task is accomplished in the following theorem, which relies on Lemmas 1 and 2.

Theorem 2 The functions $\mathbb{E}_{\eta, \widehat{f}^{B}, \widehat{g}}\left[\frac{\xi_{u}^{B}}{\xi_{t}^{B}}\right]$ and $\mathbb{E}_{\eta, \widehat{f}^{B}, \widehat{g}}\left[\frac{\xi_{u}^{B}}{\xi_{t}^{B}} \frac{\delta_{u}}{\delta_{t}}\right]$ have the following properties for positive integer risk aversions, $(1-\alpha) \in \mathbb{N}$ :

1. The effect of the variance of $\eta$ on the functions is to weakly reduce their values. In the absence of the correlation effect, ${ }^{24}$ the functions are symmetric and, in the neighborhood of $\widehat{g}=0$, are concave with respect to $\widehat{g}$.

2. When $\widehat{g} \leq 0$, the effect of the correlation between fundamental and sentiment on the two functions is nonpositive for any maturity.

3. When $u>t$, for $\widehat{g}=0$, the functions are strictly smaller for $\phi>0$ than they are for $\phi=0$.

4. In a neighborhood of $\widehat{g}=0$, the logarithmic derivatives of the functions with respect to $\widehat{g}$ are nonnegative.

5. The logarithmic derivatives of the functions with respect to $\widehat{f}^{B}$ have the sign of $(\alpha-1)$ and $\alpha$ respectively.

Proof: The proof is based on a technical device which applies when risk aversion is an integer and which constructs a bridge between the pricing measure in (32) and the moment generating function $\mathbb{E}_{\widehat{f}^{B}, \widehat{g}}^{B}\left[\left(\frac{\delta_{u}}{\delta}\right)^{\varepsilon}\left(\frac{\eta_{u}}{\eta}\right)^{\chi}\right]$. While in general the numerator of the measure (32) is a weighted power mean of the two terms corresponding to the two groups, in the special case in which risk aversion is an integer, $(1-\alpha) \in \mathbb{N}$, the pricing measure can be written in an alternative way by expanding the bracket into an exact finite sum by virtue of the binomial formula: ${ }^{25}$

$$
\left[\left(\frac{\eta_{u}}{\lambda^{A}}\right)^{\frac{1}{1-\alpha}}+\left(\frac{1}{\lambda^{B}}\right)^{\frac{1}{1-\alpha}}\right]^{1-\alpha}=\frac{1}{\lambda^{B}} \sum_{j=0}^{1-\alpha} C_{1-\alpha}^{j}\left(\frac{\eta_{u} \lambda^{B}}{\lambda^{A}}\right)^{\frac{j}{1-\alpha}},
$$

where $C_{1-\alpha}^{j}$ denotes the binomial coefficient $\left(\begin{array}{c}1-\alpha \\ j\end{array}\right)$. Therefore: ${ }^{26}$

$$
\begin{aligned}
\mathbb{E}_{\eta, \widehat{f}^{B}, \widehat{g}}^{B}\left[\frac{\xi_{u}^{B}\left(\delta_{u}, \eta_{u}\right)}{\xi_{t}^{B}(\delta, \eta)}\right]= & e^{-\rho(u-t)} H_{f}\left(\widehat{f}^{B}, t, u ; \alpha-1\right) \\
& \times[1-\omega(\eta)]^{1-\alpha} \sum_{j=0}^{1-\alpha} C_{1-\alpha}^{j}\left(\frac{\omega(\eta)}{1-\omega(\eta)}\right)^{j} H_{g}\left(\widehat{g}, t, u ; \alpha-1, \frac{j}{1-\alpha}\right),
\end{aligned}
$$

\footnotetext{
${ }^{24}$ For the definition of the correlation effect, please, refer back to Definition 1.

${ }^{25}$ The overall calculation of expectations of future discount factors is greatly simplified because the power mean has been replaced by a pseudo arithmetic mean, which allows us to use the closed-form expression for the moment generating function in (A4) when computing equilibrium prices. This is a tool that can be used to characterize equilibrium in other models with heterogeneous beliefs. Referring to the power to which $\eta$ is taken as a "degree of irrationality", this shows that, when risk aversion is $1-\alpha$, the market's average irrationality is mimicked by $(1-\alpha)+1$ degrees of irrationality, corresponding to the $(1-\alpha)+1$ terms in the summation above. Yan (2004) uses a similar approach.

${ }^{26}$ In Appendix B, we provide an alternative method for writing the pricing functions explicitly for the general case where risk aversion is not restricted to be an integer and can be any positive real number.
} 
and

$$
\begin{aligned}
\mathbb{E}_{\eta, \widehat{f}^{B}, \widehat{g}}^{B}\left[\frac{\xi_{u}^{B}\left(\delta_{u}, \eta_{u}\right)}{\xi_{t}^{B}(\delta, \eta)} \frac{\delta_{u}}{\delta}\right]= & e^{-\rho(u-t)} H_{f}\left(\widehat{f}^{B}, t, u ; \alpha\right) \\
& \times[1-\omega(\eta)]^{1-\alpha} \sum_{j=0}^{1-\alpha} C_{1-\alpha}^{j}\left(\frac{\omega(\eta)}{1-\omega(\eta)}\right)^{j} H_{g}\left(\widehat{g}, t, u ; \alpha, \frac{j}{1-\alpha}\right) .
\end{aligned}
$$

Notice how functions (43) and (44) are obtained from the moment generating function by setting, respectively, $\varepsilon=\alpha-1<0$ or $\varepsilon=\alpha \leq 0$, and summing over values of $\chi=\frac{j}{1-\alpha}, j=0, \ldots, 1-\alpha$. As a result, the validity of each and every statement of the theorem follows directly from the corresponding statement of Lemma 2.

Statement 1 shows that the risk created by the fluctuations in the expectations of others (relative to one's own) weakly reduces financial prices. ${ }^{27}$ If one were to draw the graphs of the two functions against $\widehat{g}$, they would be isomorphic to the first panel of Figure 1.

Statement 2 says that the effect of the correlation between the fundamental $\delta$ and sentiment is negative if $\widehat{g} \leq 0$. In other words, the expected values of future discount factors is further depressed when the fundamental and sentiment covary positively. If $\widehat{g}>0$, the effect of the correlation is ambiguous. The second panel of Figure 1 showed the variance and correlation effects on $H_{g}$. It was drawn against $\chi$. The two functions considered now could analogously have been graphed against $\omega$. The graph against $\omega$ would have been isomorphic to the graph of $H_{g}$ against $\chi$, as will be illustrated by the graphs for the prices of equity and bond shown below (Figure 3). ${ }^{28}$

Statement 3 shows that the effect of irrationality (that is, positive $\phi$ ) is to reduce asset prices when $\widehat{g}=0$. If $\widehat{g}>0$, however, the variations of asset prices with respect to $\phi$ are generally ambiguous. As we saw, if the irrational investors are optimistic, their optimism counteracts their irrationality.

\subsection{Securities-market implementation of the complete-market equilibrium}

There are three Wieners, $\left\{W_{\delta}, W_{f}, W_{s}\right\}$, in the economy. However, because the growth rate $f$ is not observed, only two of the three variables are observable and can be used to define "states of nature" or as a basis for writing the terms of a security's contract. Correspondingly, there are only two Wieners of consequence: $W_{\delta}^{B}$ and $W_{s}^{B}$. Therefore, three securities that are not linearly dependent are required to implement the equilibrium.

The choice of securities is largely arbitrary. We assume that there is a riskless, instantaneous bank deposit with a rate of interest $r$. "Equity" or total wealth pays the aggregate dividend $\delta$ perpetually. We introduce also a consol bond with infinite maturity paying a coupon $1 \times d t$ in each time period of length $d t .^{29}$ That makes three securities, two of which are instantaneously risky.

The equilibrium price of a fixed-income consol bond can be obtained directly from the pricing measure (32):

$$
P\left(\eta, \widehat{f}^{B}, \widehat{g}, t\right)=\frac{1}{\xi_{t}^{B}} \mathbb{E}_{\delta, \eta, \widehat{f}^{B}, \widehat{g}}^{B} \int_{t}^{\infty} \xi_{u}^{B} d u=\int_{t}^{\infty} \mathbb{E}_{\eta, \widehat{f}^{B}, \widehat{g}}^{B}\left[\frac{\xi_{u}^{B}}{\xi_{t}^{B}}\right] d u .
$$

\footnotetext{
${ }^{27}$ While the theorem pertains only to integer values of risk aversion (in which case risk aversion can only be larger than or equal to 1), one can conjecture that, whenever $\alpha<0$ (risk aversion greater than 1), an increase in the variance of sentiment reduces the expected values of all the future stochastic discount factors. But proving it would not be straightforward.

${ }^{28}$ The link between the two graphs is provided by Equations (43) and (44). In these expressions, the function $H_{g}$ is evaluated at $\chi=\frac{j}{1-\alpha}, j=0, \ldots, 1-\alpha$. As the weight $\omega$ of Group $A$ in the population increases, a higher weight $\left(\frac{\omega(\eta)}{1-\omega(\eta)}\right)^{j}$ in the summation is placed on the larger values of $\chi$. Increasing $\eta$ or $\omega$ amounts to increasing the "average" value of $\chi$ in the summation.

${ }^{29}$ We also considered finite maturity bonds and the results with these bonds instead of consols are qualitatively similar.
} 
Similarly, the equilibrium price of long-lived equity, which is also the total wealth of the economy, is the discounted sum of all future dividends:

$$
F\left(\delta, \eta, \widehat{f}^{B}, \widehat{g}, t\right)=\frac{1}{\xi_{t}^{B}} \mathbb{E}_{\delta, \eta, \widehat{f}^{B}, \widehat{g}}^{B} \int_{t}^{\infty} \xi_{u}^{B} \delta_{u} d u=\delta \int_{t}^{\infty} \mathbb{E}_{\eta, \widehat{f}^{B}, \widehat{g}}^{B}\left[\frac{\xi_{u}^{B}}{\xi_{t}^{B}} \frac{\delta_{u}}{\delta}\right] d u .
$$

The bond and equity prices are written as a summation over time of the functions (43) and (44), respectively. ${ }^{30}$ As we saw in Theorem 2, the properties of the moment generating function established earlier in Lemma 2 serve to establish properties of the prices of single-maturity assets. These properties are equally valid for perpetual assets as they were for single-maturity assets.

\section{Effect of irrationality and dispersion of beliefs on asset prices, return volatilities and risk premia}

We now study the effect of disagreement and irrationality on asset prices and their volatilities. Except for purposes of comparison, we limit ourselves to the case in which risk aversion is greater than one $(\alpha<0)$.

In order to illustrate the effect of irrationality on securities prices, we specify numerical values for the parameters of the model. Even though our objective is not to match the magnitude of particular moments in the data, we would like to work with parameter values that are reasonable. ${ }^{31}$ The parameter values that we specify are based on the estimation of a model similar to ours undertaken in Brennan and Xia (2001). In addition, we have set the volatility of the signal, $\sigma_{s}$, equal to 0.13 . The particular values chosen for all the parameters are listed in Table 1.

The dynamics of equilibrium prices are evidently determined by the state variables $\delta, \eta, \widehat{f}^{B}$, and $\widehat{g}$. Among them, the level of fundamental, $\delta$, is only a scale variable multiplying the total value of the stock market and the wealth of each group and not affecting at all the price of the bond. The state variable $\eta$ that reflects sentiment always appears in the ratio $\frac{\lambda^{B} \eta}{\lambda^{A}}$, incorporating Lagrange multipliers and the current probability measure difference between the two groups. It captures the relative Negishi weights of the two groups, with $\frac{\lambda^{B}}{\lambda^{A}}$ representing the initial (time-0) weights and the initial distribution of wealth and $\eta$ representing sentiment or the changes in the weights that have occurred as a result of the gains and losses accumulated by the irrational group. When we do not vary that ratio, we set it equal to 1 to represent the situation in which the two groups currently have similar sizes. ${ }^{32}$ But we also vary the relative weights of the two groups because we wish to study how many rational investors are needed for the market to behave almost as it would under full rationality.

As we analyze securities prices, return volatilities, and portfolio strategies, we consider the following four cases: (1) where all agents are rational $(\phi=0)$ and in agreement $(\widehat{g}=0$, or "zero dispersion of beliefs") about the growth rate of aggregate dividends; (2) where all agents are rational $(\phi=0)$ but disagree $(\widehat{g} \neq 0)$ about the growth rate; (3) where one group of agents is irrational $(\phi=0.95)$ but

\footnotetext{
${ }^{30}$ The "growth conditions" sufficient to guarantee that the time integrals in the case of perpetual securities converge (and that the interchange of the integration and expectation operators in (45) and (46) is allowed) are provided in Theorem 3 in Appendix B. Similar to the way it has been done in Brennan and Xia (2001), one can show that the condition for the equity price to converge requires that the long-run growth rate of aggregate dividends be less than the long-run risk free rate. It is worth noting that this condition depends only on the anticipated behavior of dividends, $\mathbb{E}_{\widehat{f}^{B}}^{B}\left[\left(\frac{\delta_{u}}{\delta}\right)^{\varepsilon}\right]$, and not on the anticipated behavior of sentiment, $\mathbb{E}_{\widehat{g}}^{B}\left[\left(\frac{\eta_{u}}{\eta}\right)^{\chi}\right]$, which implies that it is independent of the heterogeneity of agents in the economy.

${ }^{31}$ The range of parameter values that can be considered is restricted by the need to satisfy the growth conditions in Theorem 3, which is given in Appendix B, so that the prices of perpetual assets (equity and consol bond) are well defined. This limits, in particular, the range of values for the discount rate, or for risk aversion, that can be considered. Because of this constraint, the risk aversion we consider is somewhat too low by itself to account for the equity premium. The presence of irrational traders, however, will suffice to bring the equity premium up to realistic levels.

${ }^{32}$ If we vary the parameter $\phi$, we adjust the ratio $\frac{\lambda^{B} \eta}{\lambda^{A}}$ in such a way that the time-0 lifetime budget constraints of the two groups still hold, with unchanged time-0 endowments of securities.
} 
Table 1: Choice of parameter values and benchmark values of the state variables

\begin{tabular}{lcr} 
Name & Symbol & Value \\
\hline Parameters for aggregate endowment and the signal & $\bar{f}$ & 0.015 \\
Long-term average growth rate of aggregate endowment & $\sigma_{f}$ & 0.03 \\
Volatility of expected growth rate of endowment & $\sigma_{\delta}$ & 0.13 \\
Volatility of aggregate endowment & $\zeta$ & 0.2 \\
Mean reversion parameter & $\sigma_{s}$ & 0.13 \\
Volatility of the signal & & \\
& & \\
Parameters for the agents & $\phi$ & 0.95 \\
Agent A's correlation between signal and mean growth rate & $\lambda^{B} / \lambda^{A}$ & 0 \\
Agent B's correlation between signal and mean growth rate & $\rho$ & 0.20 \\
Agent A's initial share of aggregate endowment & $1-\alpha$ & 3 \\
Time-preference parameter for both agents & & \\
Relative risk aversion for both agents & $\delta$ & 1 \\
& $\eta$ & 1 \\
Benchmark values of the state variables & $\widehat{f}^{M}$ & $\bar{f}$ \\
The level of aggregate dividends & $\widehat{g}$ & 0 \\
The change from $B$ 's measure to $A$ 's measure &
\end{tabular}

This table lists the particular choice of parameter values used for all the figures in the paper. These values are similar to the estimation results reported in Brennan and Xia (2001). The table also indicates the benchmark values of state variables, which are the reference values taken by all state variables except for the particular one being varied in a given graph.

currently in agreement $(\widehat{g}=0)$ with the other group about the growth rate; (4) where one group of agents is irrational $(\phi=0.95)$ and disagrees $(\widehat{g} \neq 0)$ about the growth rate. Each plot in the figures below has two curves on it, with the dotted line representing the case where all agents are rational $(\phi=0)$, and the dashed line representing the case where Group $A$ is irrational $(\phi=0.95)$. Each plot has on the $x$-axis either current disagreement, $\widehat{g}$, or the relative share of aggregate consumption of the irrational Groups $A, \omega$.

\subsection{Prices}

We first consider how prices change with the average belief, $\widehat{f}^{M}$, keeping dispersion in beliefs, $\widehat{g}$, at its benchmark value of 0 , so that $\widehat{f}^{M}=\widehat{f}^{B}$. In equation (41), we saw that in our economy the rate of interest increases linearly with average belief about the growth rate, $\widehat{f}^{M}$. In other words, the yields of bonds increase with the increase in the short rate, which arises from higher expected growth. This is because higher growth of dividends implies lower marginal utility of future consumption. In the case of equity, the same effect is present and, when risk aversion is greater than 1, that effect dominates the effect of increased future dividends. ${ }^{33}$ Thus, the ratio of the price of equity to current dividends drops, as did the price of the bond, with an increase in average belief of future growth. For analytical confirmation, see Statement 5 of Theorem 2.

\footnotetext{
${ }^{33}$ That is one way in which our model differs from those of Brennan and Xia (2001) and David (2004). When Brennan and Xia increase their investors' estimate of expected dividend growth, they keep constant their investors' estimate of expected aggregate consumption growth. The ratio of the price of equity to current dividend rises. When increasing their investors' estimate of expected aggregate consumption while keeping expected dividend growth constant, the price of equity drops. In our model, consumption equals dividend. When investors become more optimistic about future dividend growth, if risk aversion is greater than 1 then the second effect dominates, and hence, the price-dividend ratio drops.
} 
Figure 2: Equity and bond prices against dispersion of beliefs
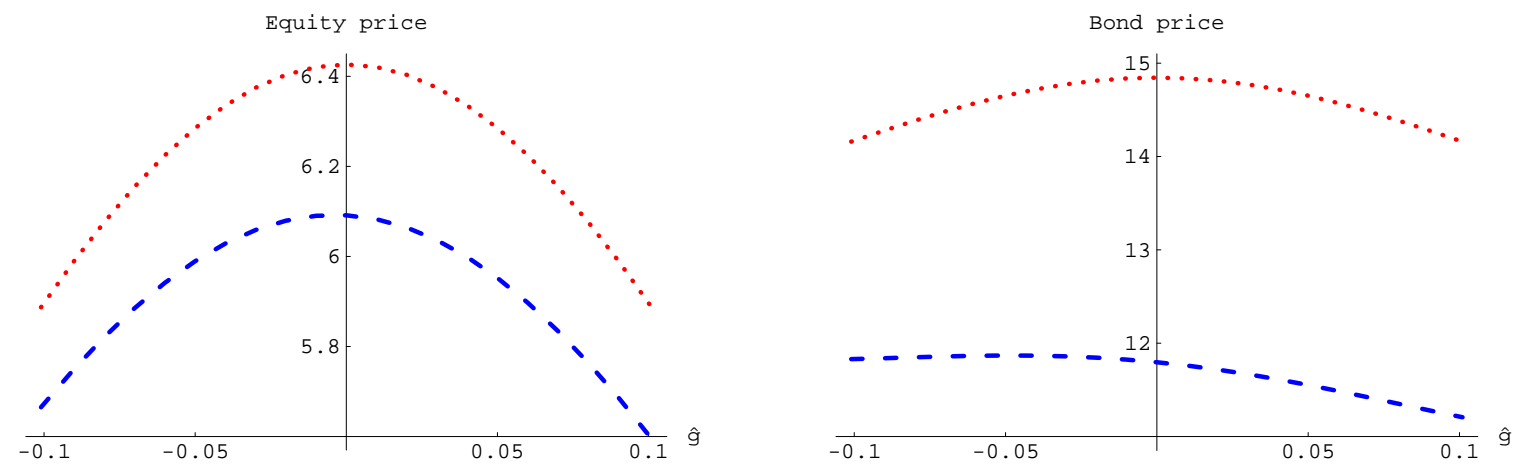

The prices of equity (panel on the left) and bonds (panel on the right) are plotted as functions of dispersion of beliefs, $\widehat{g}$, for $\phi=0$ (higher dotted curve) as well as $\phi=0.95$ (lower dashed curve). The parameter values used here are given in Table 1. In particular, the two groups of investors have equal weight and average expectation, $f^{M}$, is held fixed at $\bar{f}$.

We now turn to the role of the dispersion of beliefs. When varying the dispersion of beliefs, $\widehat{g}$, keeping other variables, especially average belief $\widehat{f}^{M}$, at their benchmark values, we recall that the rate of interest is a quadratic function of $\widehat{g}$ that is symmetric around $\widehat{g}=0$. Almost as a mirror image of the rate of interest, the prices of the bond and of equity decline in Figure 2 as the square of $\widehat{g}$ increases. These variations are due to the fact that disagreement $\widehat{g}$ translates into a larger variance of the sentiment $\eta$. We pointed out in Subsection 4.2 that state prices are concave functions of sentiment so that, by Jensen's inequality, the expected value of their future value is reduced when the variance of $\eta$ increases. Disagreement is a genuine sources of risk in the marketplace, with negative consequences for prices. The same point was made rigorously in Statement 1 of Theorem 2 and, of course, the same effect is present in perpetual asset prices. When some investors in the population are irrational, there is a further drop in asset prices (dashed line for $\phi \neq 0$ in Figure 2), which can be traced back to Statements 3 of Theorem 2 and Lemma $2 .{ }^{34}$

Next, we study the effect on asset prices of a change in the weight $\omega(\eta)=c^{A} / \delta$ of the irrational Group $A$ in the population, keeping the average belief and dispersion of beliefs at their benchmark values $\left(\widehat{f}^{M}=\bar{f}\right.$ and $\left.\widehat{g}=0\right)$. The result for equities and bonds is shown in Figure 3. The dotted horizontal line shows that, not surprisingly, if everybody is rational and there is no disagreement, the relative weight of the two groups has no effect on asset prices. The solid curve is drawn for the case where only the effect of variance of sentiment $(\eta)$ matters and the effect of correlation between the fundamental and sentiment is zero. ${ }^{35}$ As described in Theorem 2, the variance effect is unambiguously negative. Consequently, asset prices drop. The minimum prices for this case obtain when the degree of heterogeneity is highest, $\omega=0.5$. Finally, the dashed curve shows the price when one accounts also for the effect of correlation between the fundamental, $\delta$, and sentiment, $\eta$. As discussed in Statement 2 of Theorem 2, when $\widehat{g}=0$, this correlation effect is also negative, and so leads to a further drop in asset prices. ${ }^{36}$

The correlation effect also accounts for the fact that if the share of the irrational group approaches 1 and $\widehat{g}=0$, prices of all securities are lower than in the pure rational case (that is, in the neighborhood of $\omega=1$ in Figure 3, the dashed line for the case with irrationality is below the dotted line for the case with rationality). ${ }^{37}$ The replacement of $B$ 's measure with $A$ 's measure is the only difference between the security price levels for these two extreme values of $\omega$. It amounts to replacing $\widehat{f}^{B}$ with $\widehat{f}^{A}$, because the two groups have different beliefs about future growth, and simultaneously replacing $\gamma^{A}$ with $\gamma^{B}>\gamma^{A}$,

\footnotetext{
${ }^{34}$ If $\omega=0.5$, the price functions for equity and the bond are symmetric under rational, that is, pure Bayesian learning (dotted line), where $\phi=0$, and disagreement can arise only from differences in priors. However, they are not symmetric when Group $A$ is irrational. As has been mentioned in Footnote 22, this is because the concepts of average belief $\widehat{f}^{M}$ and dispersion of beliefs $\widehat{g}$ are only applicable, strictly speaking, to the immediate maturity and not to other maturities.

${ }^{35}$ For the definition of the correlation effect, please, refer back to Definition 1 and to Equation (22).

${ }^{36}$ The reason for which Figure 3 mirrors the second panel of Figure 1 was given in Footnote 28.

${ }^{37}$ In this respect also, Figure 3 mirrors the second panel of Figure 1.
} 
Figure 3: Equity and bond prices against Group A's consumption share
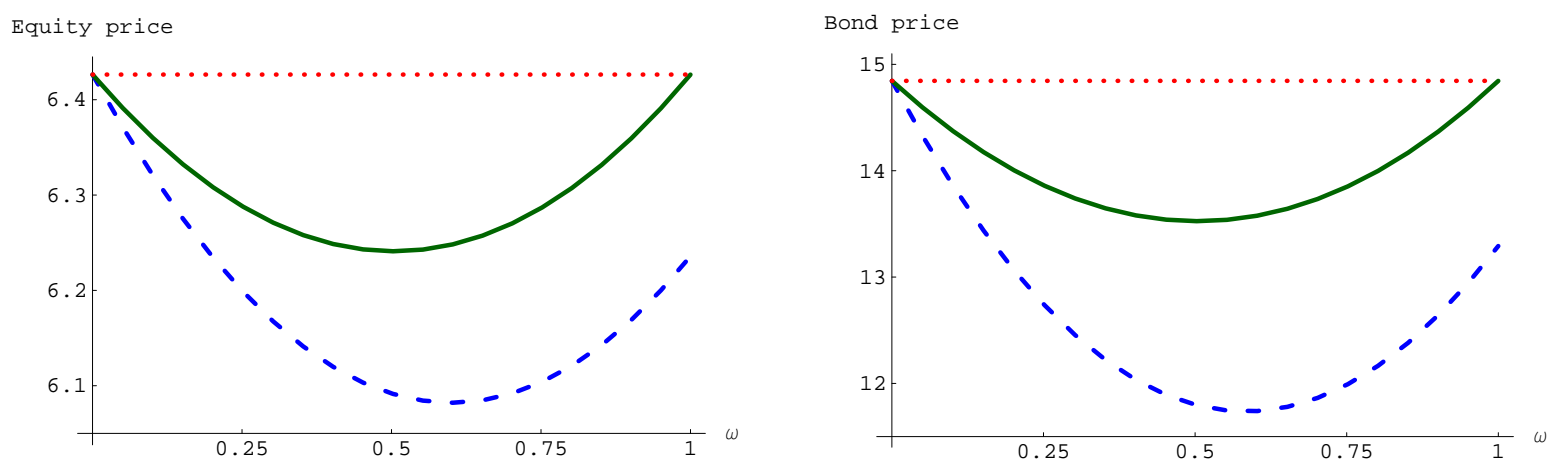

The prices of equity and bonds are plotted as functions of $\omega$, the consumption-share of Group $A$, for $\phi=0$ (higher dotted line) as well as $\phi=0.95$ (lower dashed curve and intermediate solid curve). The intermediate, solid curve represents the pure effect of sentiment fluctuations on price. The difference between the solid and the dashed lines is the effect of the covariation between the fundamental and sentiment. The parameter values and benchmark values used here are given in Table 1. In particular, the plots are drawn for the case of zero dispersion of beliefs, $\widehat{g}=0$ and average expectation, $f^{M}$, is held fixed at $\bar{f}$.

because the two groups do not treat the signal the same way and Group $A$ is overconfident about their conditional estimate of growth (see Footnote 11). The first effect is absent in our figure because we are keeping $\widehat{g}=0$. So, the second effect, the difference between $\gamma^{B}$ and $\gamma^{A}$ is the reason for the price gap between the rational solution (dotted line) and the irrational solution (dashed line) in the neighborhood of $\omega=1$. By being overconfident conditionally, Group $A$ creates noise, in the form of additional unconditional or long-run $\widehat{g}$ risk, which in turn increases $\eta$ risk, which itself is priced in the market.

We summarize our findings about the levels of securities prices in the two cases of rationality and irrationality in the proposition below. Comparing the levels of dashed curves to those of dotted curves in Figures 2 and 3 illustrates that:

Proposition 2 In a neighborhood of zero dispersion of beliefs $(\widehat{g}=0)$ and provided risk aversion is greater than $1(\alpha<0)$ : (i) the price levels of equity and bonds are reduced by the presence of irrational traders, and (ii) there exists a value of the weight of the irrational investor group in the market such that, above that value, the prices of equity and the bond are lower than they would have been had either group been alone in the economy.

The reason for the above result is that irrational traders add "noise" for which all traders require a risk premium. We have shown that "noise traders create their own space" in the sense of De Long, Shleifer, Summers, and Waldmann (1990a). Because we get this result in a general equilibrium setting, our analysis is not subject to the criticism of Loewenstein and Willard (2005).

Harrison and Kreps (1978) provide an example of a speculative-behavior equilibrium in which asset prices are above the valuation of all agents. In their model, investors are risk neutral and are not allowed to sell short. ${ }^{38}$ In such a setting, when a stock is perceived to be overvalued, agents cannot sell it short. Hence, they go along with the overvaluation and hold the stock positively because there is a chance that a category of investors will grow to be very optimistic in the future and will want to buy the stock. The model in Scheinkman and Xiong (2003) is an example of a dynamic equilibrium of that type. The "option to resell" adds value to the stock over and above the value it has in the eyes of the current owners.

In our setting, the mechanism driving prices away from their fundamental value is different from that in Harrison and Kreps. Investors are not risk neutral and we do not constrain them from selling

${ }^{38}$ For the analysis of the effect of short selling on excess volatility, see Gallmeyer and Hollifield (2004). 
short. Instead, investors limit the portfolio positions they take naturally as a consequence of being risk averse. In fact, in the setting we consider, no investor would pay for the option to sell at a future market price because they could simply sell short without cost. Moreover, in our model, both categories of investors generally hold the stock simultaneously. So, in our model, it is possible for today's valuation to be either above or below what it would be if each category of investors had to value the anticipated dividend stream based on its own expectations process. If risk aversion is greater than 1 and there is no disagreement, prices can only be below the private valuation of the rational investors. The prices are also below the private valuation of irrational investors provided the weight of the rational group is not too large; that is, in Figure 3 the lowest prices are attained not for the case where $\omega=1$ but when there is heterogeneity in the population. Had we, instead, assumed that risk aversion is less than 1, thereby getting closer to the Harrison-and-Kreps case of risk neutrality, prices in our model could very well have been above the private valuation of rational investors. ${ }^{39}$

\subsection{Return diffusions, volatilities and correlation}

In this subsection, we study the volatilities of equity and bond returns, and also the correlation between equity and bond returns. The volatilities are obtained from the diffusion vectors (sensitivities or exposures to the innovation in the fundamental, $d W_{\delta}$, and in the signal, $d W_{s}$ ) of the prices of equity and the bond, which result from a straightforward application of Itô's lemma to the explicit expressions for the value of equity and bond. They are equal to the gradient of the price function premultiplying the diffusion matrix of state variables (Equation (18)). Generally, equity is positively exposed to the realized innovation in the fundamental, $d W_{\delta}$, whereas the bond is negatively exposed. The reason for this is that a shock to the fundamental affects equity in two ways: it changes the payoff and also the valuation operator. On the other hand, for bonds an innovation in the fundamental has only a valuation effect, which is negative because it is harbinger of larger future output. The signal innovation, $d W_{s}$, is similar but it has no immediate payoff implication, so that both the equity and the bond have a negative exposure to it.

As we saw in the previous section, the levels of securities prices are typically reduced when the "irrationality parameter" $\phi$ is taken from $\phi=0$ to $\phi=0.95$. The effect of the irrationality of Group $A$ is also to increase the volatility of asset prices. This occurs because of the greatly increased volatility of the state variable $\widehat{g}$ and, because of it, also from the increase in the volatility of $\eta$. Figure 4 plots the volatilities of the rate of return on equity and the bond, and the correlation between them. As before, in the first column of the figure, each quantity is plotted against dispersion of beliefs, $\widehat{g}$, in the two cases of irrationality and rationality. Irrational investors create "noise" that increases the volatility of both risky assets - the stock and the bond. The volatility increases without bounds when there is disagreement $(\widehat{g} \neq 0)$. The values produced by the model for the volatility of bond returns (and interest rates) are regrettably too high to fit real-world data. ${ }^{40}$

The last plot in the first column of Figure 4 shows that the correlation between stock and bond returns also increases with the presence of overconfident investors, as well as with disagreement between the two investor groups. This is because, as we pointed out in the previous section, the prices of the equity and bond move in the same direction when expectations fluctuate. The correlation increases under irrationality but agreement $(\widehat{g}=0)$ and it approaches 1 as disagreement is introduced $(\widehat{g} \neq 0)$.

To determine how the level of volatility in the market varies with the relative weight of Groups $A$ and $B$, we plot in the second column of Figure 4 the volatilities of the equity and bond returns and their correlation as a function of Group $A$ 's share of aggregate consumption, $\omega$. These plots shows that the excess volatility in the market increases with an increase in the relative weight of the irrational Group $A$.

\footnotetext{
${ }^{39} \mathrm{~A}$ similar phenomenon was discovered by Cao and Ou-Yang (2005). It would appear that their results are based mostly on the effect of the covariance between $\eta$ and $\delta$ (in our notation). Each investor faces the prospect of the other investor becoming more or less optimistic than he/she is today and incorporates that prospect in the price he/she is willing to pay today. Our conclusion is that the prospect of the other investor becoming less optimistic dominates whenever risk aversion is greater than one.

${ }^{40}$ With a risk aversion smaller than 1, David (2004) was able to match the volatility of interest rates much better. Alternatively, if one wanted to match interest-rate volatility, one could introduce habit formation.
} 


\section{Figure 4: Volatilities of equity and bond returns and their correlation}
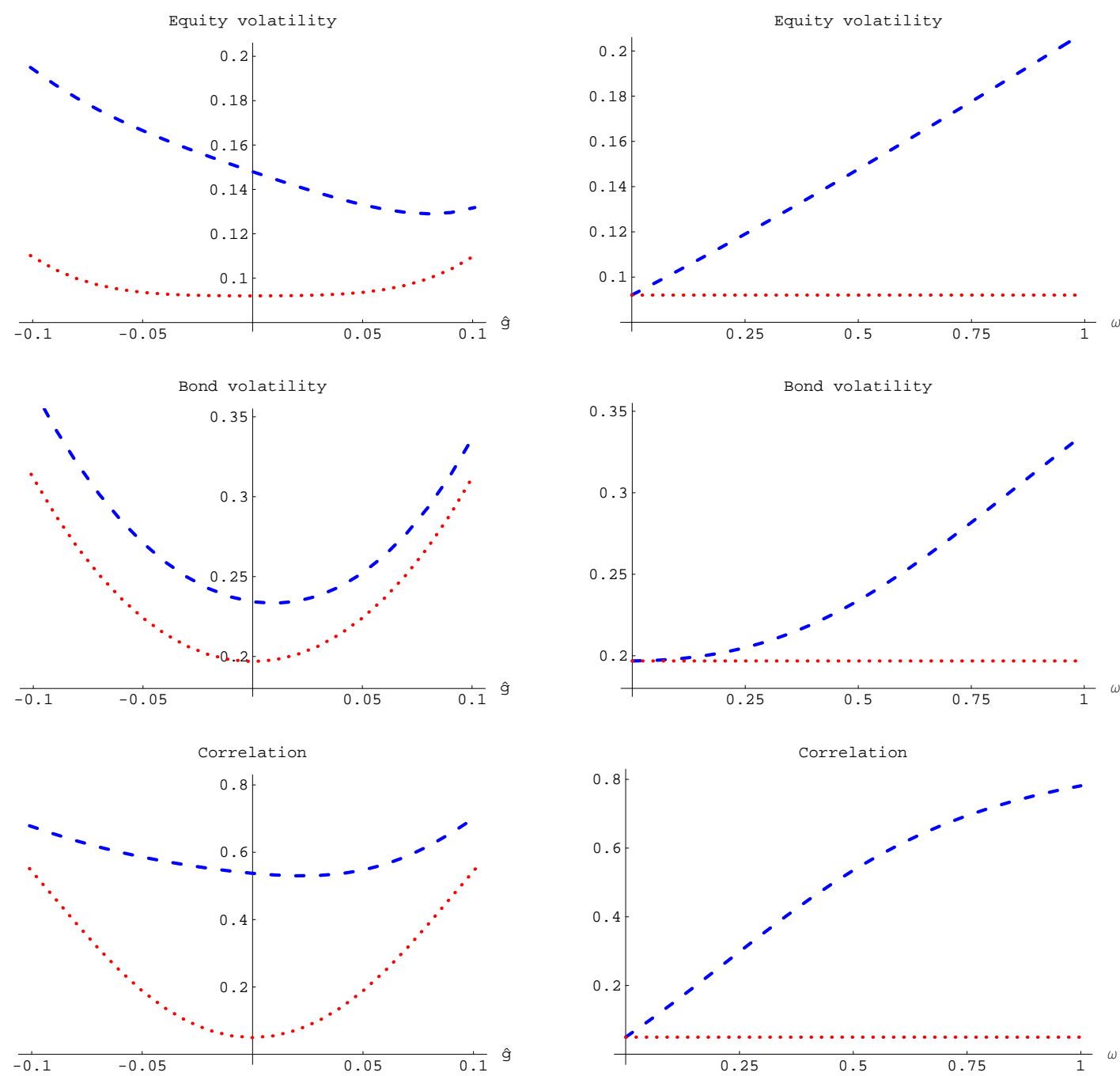

The first column of this figure plots against dispersion in beliefs, $\widehat{g}$, the volatilities of equity and bond returns and their correlation, assuming that the two groups of investors have equal weight, $\omega=0.5$. In the second column of this figure, the same quantities are plotted but this time against the relative weight of Group $A$ in the population, $\omega$, assuming that $\widehat{g}=0$. There are two curves in each plot: the dotted curve is for the case of rationality $(\phi=0)$ and the dashed curve is for the case of irrationality $(\phi=0.95)$. In all the plots, average expectation, $f^{M}$, is held fixed at $\bar{f}$. All other parameter values used in this figure are given in Table 1.

More importantly, these plot show that it is not enough to have just a few rational investors to get the volatility down to the level warranted by fundamentals; in fact, the first plot in the second column shows that the equity return volatility decreases only linearly as we increase the relative weight of rational investors in the population.

We summarize these results about second moments in the proposition below.

Proposition 3 Irrational investors create "noise" that increases the volatility of both stock and bond returns and also the correlation between them. The volatilities and correlation increase without bounds when there is disagreement $(\widehat{g} \neq 0)$. The volatilities and correlation increase also with an increase in the relative weight in the population of Group A. 
Figure 5: Expected excess returns for equity and bond
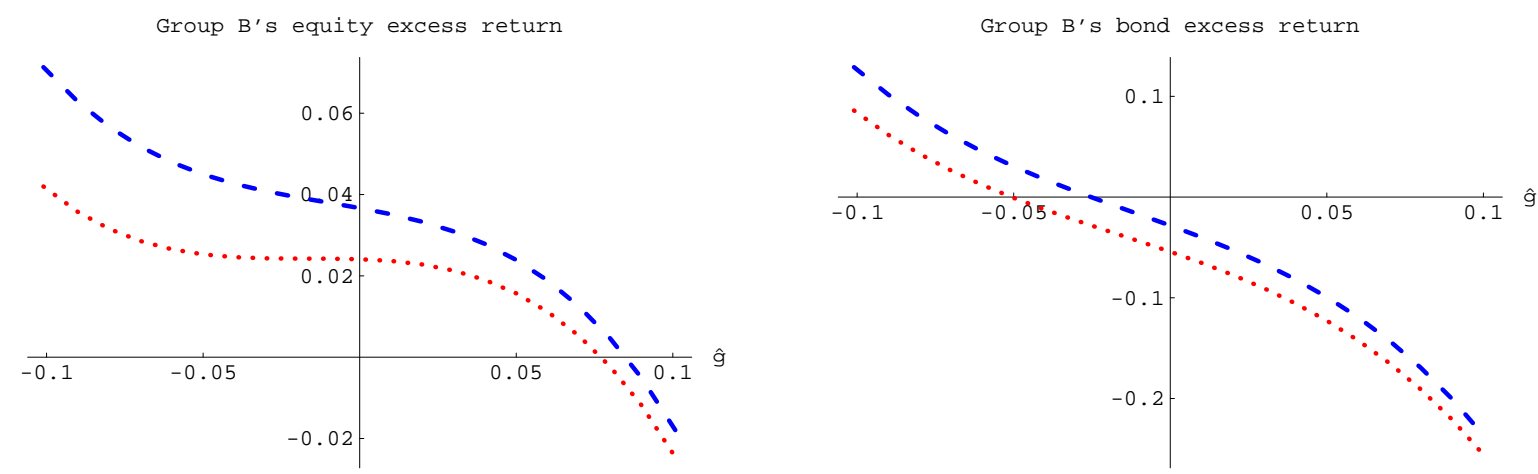

The expected excess returns on equity and bond are plotted against $\widehat{g}$, for $\phi=0$ (dotted line) as well as $\phi=0.95$ (dashed curve). The parameter values used here are given in Table 1. In particular, the two groups of investors have equal weight and average expectation, $f^{M}$, is held fixed at $\bar{f}$.

\subsection{Conditionally expected excess returns}

A final determinant of Group $B$ 's portfolio choice will be the excess rates of return conditionally expected by them. From the first plot of Figure 5, we see that with enough disagreement and/or irrationality, it is quite easy to account for a high equity risk premium, especially when rational investors are currently relatively pessimistic $(\widehat{g}<0)$. The expected excess return on bonds is typically negative. The reason for this, as explained in the section below that describes the portfolio choice of investors, is that the demand for bonds is very high because they serve as a useful security for intertemporal hedging. Thus, in equilibrium, investors are willing to hold bonds even when the expected return on bonds is less than that on the instantaneously riskless security.

An additional observation is that expected excess returns are not independently, identically distributed over time. Expected returns are very much affected by the current values of state variables, a situation commonly referred to in the Finance literature as "return predictability". In this model, however, return predictability is not as simple as has been commonly envisaged in the empirical Finance literature. For instance, there exists sometimes a positive relation between expected return on equity and the dividend yield and sometimes a negative one.

\section{The optimal portfolio of rational Group $B$}

In this section, we study the fluctuations of the wealth of Group $B$ and deduce from it the main features of the portfolio strategy of the rational investors.

The wealth of agents of Group $B$ can be determined by applying the same approach that we used to find the equity and bond prices. To do so, we interpret the wealth of agents of Group $B$ as the price of a "security" whose flow payoff at future time $u$ is the consumption (34) of these investors:

$$
F^{B}\left(\delta, \eta, \widehat{f}^{B}, \widehat{g}, t\right)=\frac{1}{\xi_{t}^{B}} \mathbb{E}_{\delta, \eta, \widehat{f}^{B}, \widehat{g}}^{B} \int_{t}^{\infty} \xi_{u}^{B} c_{u}^{B} d u=\delta[1-\omega(\eta)] \int_{t}^{\infty} \mathbb{E}_{\eta, \widehat{f}^{B}, \widehat{g}}^{B}\left[\frac{\xi_{u}^{B}}{\xi_{t}^{B}} \frac{c_{u}^{B}}{c_{t}^{B}}\right] d u,
$$

where the price of the single-maturity claim to Group B's consumption,

$$
\begin{aligned}
\mathbb{E}_{\eta, \hat{f}^{B}, \widehat{g}}^{B}\left[\frac{\xi_{u}^{B}\left(\delta_{u}, \eta_{u}\right)}{\xi_{t}^{B}(\delta, \eta)} \frac{c_{u}^{B}\left(\delta_{u}, \eta_{u}\right)}{c_{t}^{B}(\delta, \eta)}\right]= & e^{-\rho(u-t)} H_{f}\left(\widehat{f}^{B}, t, u ; \alpha\right) \\
& \times[1-\omega(\eta)]^{-\alpha} \sum_{j=0}^{-\alpha} C_{-\alpha}^{j}\left(\frac{\omega(\eta)}{1-\omega(\eta)}\right)^{j} H_{g}\left(\widehat{g}, t, u ; \alpha, \frac{j}{1-\alpha}\right),
\end{aligned}
$$


is obtained using the binomial expansion described in Equation (42). Therefore, the properties delineated in Theorem 2 hold for function (48) as well.

Following Cox and Huang (1989), the portfolio composition of Group $B$ in terms of actual securities can be calculated from Group B's demand for exposure to shocks to the state variables, which are themselves obtained by multiplying the gradient vector of $B$ 's wealth by the diffusion matrix of state variables given in Equation (18). In order to interpret the optimal portfolio holdings, we first put to use, in Subsection 6.1, the terminology introduced in Merton (1973) to explain when investors view shocks to the fundamental, $d W_{\delta}$, and innovations in the signal, $d W_{s}$, as favorable or unfavorable shifts in their investment opportunity set. The optimal exposure demanded by agents to these two shocks is derived in Subsection 6.2. In Subsection 6.3, we show how to construct "elementary contracts" that create a unit of exposure to each of the shocks. When an investor holds an elementary contract creating a unit of exposure to shocks to the fundamental, for instance, he or she receives a stream of payoff flow, $d W_{\delta, u}$ time after time. The market price of such elementary contracts, being the expectation of a stochastic integral relative to Wieners, is zero. Finally, in Subsection 6.4 we show how to implement the optimal strategy of Group $B$ using the stock, bond and riskless asset.

\subsection{Favorable vs. unfavorable shifts in a person's investment opportunity set}

Exposure demands are the direct consequences of the gradient of the wealth function, $F^{B}\left(\delta, \eta, \widehat{f}^{B}, \widehat{g}, t\right)$. A state variable has an impact on Group $B$ 's wealth for two possible economic reasons: (i) it can change the prospect for the immediate return on a given portfolio held by Group $B$, and (ii) it can affect the investment opportunities in the future that Group $B$ will face when rebalancing their portfolio. Were the variable $\xi^{B}(\delta, \eta)$ an argument of $F^{B}$, it would capture entirely the first effect. Indeed, Cox and Huang (1989) have shown that the derivative $\frac{\partial F^{B}}{\partial \xi^{B}}$ is the basis for the "static" component of portfolio demand, corresponding to pure "Markowitz" one-period portfolio choice. In order to segregate the two effects, one can introduce the following change of variable:

$$
\widetilde{F}^{B}\left(\xi^{B}, \eta, \widehat{f}^{B}, \widehat{g}, t\right) \equiv F^{B}\left(\delta, \eta, \widehat{f}^{B}, \widehat{g}, t\right) .
$$

Then, given that $\frac{\partial F^{B}}{\partial \delta}>0$ and $\frac{\partial F^{B}}{\partial \eta}<0$, it is easy to show that $\frac{\partial \widetilde{F}^{B}}{\partial \xi^{B}}<0$. In fact,

$$
\frac{1}{F^{B}} \frac{\partial \widetilde{F}^{B}}{\partial \xi^{B}}=-\frac{1}{1-\alpha} \frac{1}{\xi^{B}}<0
$$

This derivative will determine what we call the static component of exposure demand that is driven by immediate returns.

The interpretation of the effect of Type (ii) can be based on the concept of "favorable or unfavorable shift in the investment opportunity set" introduced by Merton (1973), where "a favorable shift" is defined as a change in a state variable such that consumption rises for a given level of wealth. In the present context, the nature of a shift is revealed by the sign of the derivative of the wealth function $\widetilde{F}^{B}$ for a given level of $\xi^{B}{ }^{41}$ The gradient of the wealth function (47) is obtained from the derivatives of the moment generating function (for the signs of the derivatives, $c f$. Statements 4 and 5 of Theorem 2).

\footnotetext{
${ }^{41}$ Denote by $J\left(F^{B}, Y\right)$ the value function of the investor's lifetime utility under a Merton-like dynamic-programming formulation of the investor's optimization problem, where $F^{B}$ denotes the investor's wealth and $Y$ any state variable. Then: $\xi^{B}=\frac{\partial J}{\partial F^{B}}\left(\widetilde{F}^{B}(\xi, Y), Y\right)$, which can be differentiated with respect to $Y: 0=\frac{\partial^{2} J}{\partial\left(F^{B}\right)^{2}} \times \frac{\partial \widetilde{F}^{B}}{\partial Y}+\frac{\partial^{2} J}{\partial F^{B} \partial Y}$ implying that $\frac{\partial \widetilde{F}^{B}}{\partial Y}=-\frac{\partial^{2} J}{\partial F^{B} \partial Y} / \frac{\partial^{2} J}{\partial\left(F^{B}\right)^{2}}$. From the concavity of the utility function, we know that $\frac{\partial^{2} J}{\partial\left(F^{B}\right)^{2}}<0$ so that $\frac{\partial F^{B}}{\partial Y}$ has the same sign as $\frac{\partial^{2} J}{\partial F^{B} \partial Y}$. Merton (1973) defines "a favorable shift" as a positive shock to a state variable $Y$ that has the property that $\frac{\partial^{2} J}{\partial F^{B} \partial Y}<0$, because that implies that a positive shock to $Y$ induces a decrease in marginal utility and a rise in consumption. So, in terms of wealth, a positive shock to a variable $Y$ with the property that $\frac{\partial F^{B}}{\partial Y}<0$ is viewed as a favorable shift.
} 
Because $\xi^{B}$ depends on the fundamental, $\delta$, and sentiment, $\eta$, but not on $B$ 's belief about the expected growth rate, $\widehat{f}^{B}$, and the dispersion in beliefs, $\widehat{g}$, the last two variables can have only effects of Type (ii). As was the case for equity, or total wealth of the economy, the effect on the wealth of Group $B$ of a rise in the growth anticipated by themselves $\left(\hat{f}^{B}\right)$ is to decrease wealth. This means that a positive random shock on $\widehat{f}^{B}$ is a "favorable shift" for Group $B$ (as well as for Group $A$ ): in states of nature in which their growth expectation is high, investors have arranged to have less accumulated savings to finance future consumption. That is, when choosing its portfolio, Group $B$ seeks to hold securities with high unexpected returns in states of nature that have negative shocks to $\widehat{f}^{B}$. Subsections 6.3 and 6.4 will reveal that bonds have that property.

The derivative of the wealth function (Equation 47) with respect to dispersion in beliefs, $\widehat{g}$, is nonnegative. ${ }^{42}$ This means that, in a neighborhood of no disagreement, a positive random shock in $\widehat{g}$, which renders Group $A$ more pessimistic than Group $B$, is an "unfavorable shift" for Group $B$. Consequently, when making up its portfolio, Group $B$ will seek to protect itself against future positive shocks to $\widehat{g} .{ }^{43}$

\subsection{Exposure demand of Group $B$}

In this subsection, we analyze Group $B$ 's equilibrium levels of demand for elementary contracts which are equal to the gradient of $B$ 's wealth multiplied by the diffusion matrix of the state variables $\left\{\xi^{B}, \eta, \widehat{f}^{B}, \widehat{g}\right\}$ :

$$
\left[\begin{array}{llll}
\xi^{B} \frac{\partial \widetilde{F}^{B}}{\partial \xi^{B}} & \eta \frac{\partial \widetilde{F}^{B}}{\partial \eta} & \frac{\partial \widetilde{F}^{B}}{\partial \widehat{f}^{B}} & \frac{\partial \widetilde{F}^{B}}{\partial \widehat{g}}
\end{array}\right] \cdot\left[\begin{array}{cc}
-(1-\alpha) \sigma_{\delta}-\widehat{g} \omega(\eta) \frac{1}{\sigma_{\delta}} & -\widehat{g} \omega(\eta) \frac{1}{\sigma_{s}} \\
-\frac{\widehat{g}}{\sigma_{\delta}} & -\frac{\widehat{g}}{\sigma_{s}} \\
\frac{\gamma^{B}}{\sigma_{\delta}}>0 & \frac{\gamma^{B}}{\sigma_{s}}>0 \\
\frac{\gamma^{B}-\gamma^{A}}{\sigma_{\delta}} \geq 0 & \frac{\gamma^{B}-\left(\phi \sigma_{s} \sigma_{f}+\gamma^{A}\right)}{\sigma_{s}} \leq 0
\end{array}\right] .
$$

Exposure demands as percentages of the total wealth of Group $B$ are plotted in the first row of Figure 6 against the current level of disagreement $\widehat{g}$, in the two cases of rationality and irrationality, holding average expectation $f^{M}$ fixed at $\bar{f}$. ${ }^{44}$ The first plot in the top row shows that Group $B$ wants to be positively exposed to fundamental shock (unless they are very pessimistic) and the second plot shows that Group $B$ wants to be negatively exposed to signal shock (unless they are very optimistic). ${ }^{45}$ Observe also that Group B's exposures to both shocks increase as they become more optimistic $(\widehat{g}$ increases). Both statements are true in the case of rationality and agreement as well as in the case of irrationality. Moreover, in the case of irrationality, the positive exposure to $\delta$ shocks and the negative exposure to signal shock are both increased in size by approximately $50 \%$ (in the neighborhood of agreement), compared to the case of rationality.

The scales on the vertical axes of Figure 6 should not mislead the reader. For instance, under rationality and agreement, Group $B$ demands an exposure to the fundamental shock under rationality which is about $5 \%$ of their wealth and an exposure to signal shock which is about $-7 \%$. The size of the number does not mean that these are small amounts of risk. Recall that the shocks are standard Brownians with a standard deviation equal to 1 . So a $100 \%$ exposure to one of these risks would reflect a desire to hold a portfolio that has a $100 \%$ standard deviation per year! When we implement these exposures by means of equity and bonds, we shall have occasion to verify that investors put most of their wealth into risky equity.

\footnotetext{
${ }^{42}$ In Statement 4 of Lemma 1, we saw that, around $\widehat{g}=0$, with $\varepsilon=\alpha<0$, the derivative $\frac{1}{H_{g}} \frac{\partial H_{g}}{\partial \widehat{g}}$ is nonnegative, as evidenced in the third panel of Figure 1. The same is true also for the single-payoff claims characterized in Statement 4 of Theorem 2. This property carries over also to the wealth function.

${ }^{43}$ However, as can be seen from the third panel of Figure 1, and verified analytically from the second term in the derivative (A49), for large enough positive $\widehat{g}$, the effect of the variance of $\eta$ can dominate and change the sign of the derivative $\partial F^{B} / \partial \widehat{g}$ to turn a positive random shock in $\widehat{g}$ from an unfavorable shift into a favorable shift.

${ }^{44}$ Recall, however, the caveat of Footnote 22: holding $\widehat{f}^{M}$ fixed holds average belief fixed for only the immediate maturity. For other maturities, this is only a convenient but approximate interpretation device.

${ }^{45}$ Elementary contracts require no financing since their price is zero. Besides entering into these contracts, investors put all their wealth into the riskless security.
} 
Figure 6: Group B's demand for exposures to fundamental shocks and signal shocks
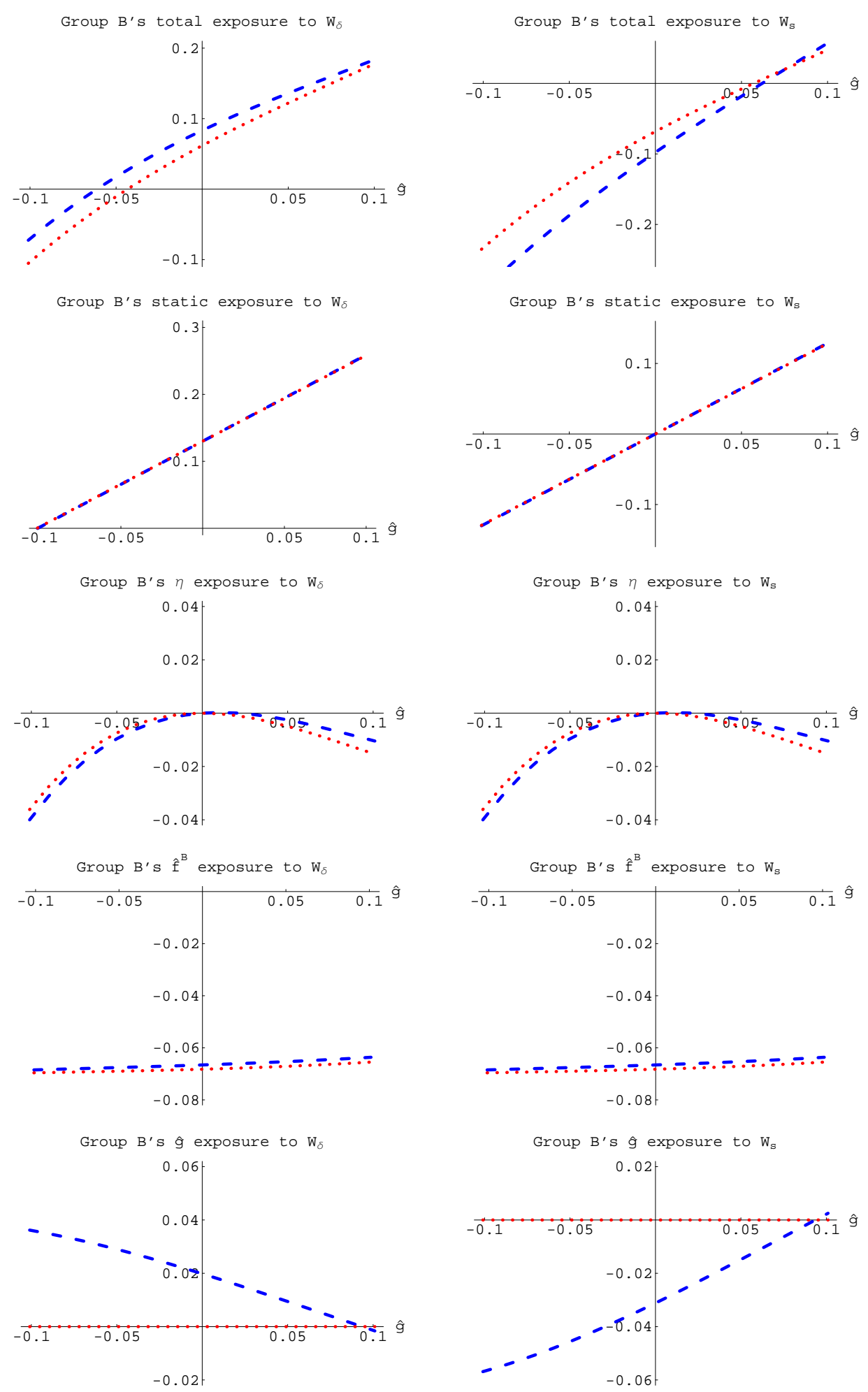

Group B's equilibrium levels of demand for exposures to the fundamental and signal shocks, $d W_{\delta}$ and $d W_{s}$, are plotted in the first row against $\widehat{g}$, for $\phi=0$ (dotted line) and $\phi=0.95$ (dashed line). Rows 2 to 5 of the figure decompose the exposure demands into a static component (driven by immediate returns) and intertemporal hedging components. The parameter values used in these plots are given in Table 1. In particular, average expectation, $f^{M}$, is held fixed at $\bar{f}$. 
In order to explain the motives behind these exposure demands, we now break up the total exposures to the fundamental and signal innovation into four pieces, each corresponding to one of the elements of the gradient of $\widetilde{F}^{B}$. We give a name to each piece of the product (51): the static exposure, the $\eta$ exposure, the $\widehat{f}^{B}$-exposure and the $\widehat{g}$-exposure. Rows 2 to 5 of Figure 6 show each of these components of total exposure. ${ }^{46}$

Two clear conclusions emerge from Rows $2-5$ of Figure 6 . First, the only components of exposure demand that are markedly affected by the presence of irrationality are the $\widehat{g}$-exposures. Indeed, under rationality, $\widehat{g}$, as we have seen, has zero diffusion so that the exposures are zero. Under irrationality, they become sizable because of the last row of the diffusion matrix, which is where the influence of $\phi$ makes itself felt (with the signs indicated). Because $\gamma^{B}-\gamma^{A} \geq 0$, the rational traders know that a positive $d W_{\delta}$ shock will increase disagreement (see also Equation (17)) which, as we have seen, is an unfavorable shift for them, at least in a neighborhood of $\widehat{g}=0$. Hence, they construct their portfolio to have a positive exposure to $d W_{\delta}$, as an offset. Because $\gamma^{B}-\left(\phi \sigma_{s} \sigma_{f}+\gamma^{A}\right) \leq 0$, the opposite is true for the signal shock.

Second, the static exposures are distinctly not affected by irrationality; that is, in the second row of Figure 6, the dotted line for $\phi=0$ and the dashed line for $\phi=0.95$ are identical. This result can be demonstrated by multiplying the partial log-derivative with respect to $\xi^{B}$ in (50) by the diffusion of $\xi^{B}$ (which is $-\xi^{B}\left(\kappa^{B}\right)^{\top}$ as defined in (38)) and observing that the product does not involve $\phi$ :

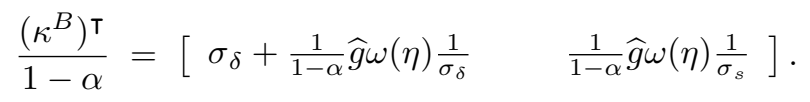

We know from Cox and Huang (1989), that the partial derivative with respect to $\xi^{B}$ gives the exposure to $\xi^{B}$, which is precisely the static (Markowitz) component. This is because the diffusion vector of $\xi^{B}$ is equal to the vector of expected returns premultiplied by the diffusion matrix of securities returns, which means that the static exposure serves to exploit return predictability. Clearly, exploiting the return predictability created by irrationality is not a concern of Group B's portfolio strategy. However, it is also clear that, under irrationality as well as under rationality, the static exposures are affected by disagreement: as Group $B$ grows more optimistic than Group $A$, it greatly increases its exposure to both shocks. The divergent opinion of Group $A$ is, in the eyes of Group $B$, a source of return predictability because, as we have seen, disagreement is mean reverting.

We summarize our conclusions in the proposition below.

Proposition 4 The only components of exposure demand that are markedly affected by the presence of irrationality are the exposures to the dispersion in beliefs (that is, the $\widehat{g}$-exposures). Exploiting the return predictability created by irrationality is not a concern of Group B's portfolio strategy, while exploiting the return predictability created by disagreement is an important concern.

\subsection{Implementation: Constructing elementary contracts}

The elementary contracts that were demanded by investors in Subsection 6.2 are the key to the implementation of the "risk-arbitrage" strategy based on excessive volatility. These elementary contracts can be viewed as zero market value derivatives or contingent debt-equity swaps that can be replicated by combining equity, the bond and the riskless asset. Replicating them is a standard exercise in financial engineering. The replicating portfolio is obtained by inverting the diffusion matrix of the risky returns of equity and the bond. The result is displayed in Figure 7.

\footnotetext{
${ }^{46} \mathrm{It}$ is easy to see how each row arises from the multiplication of each derivative by the corresponding row of the diffusion matrix of state variables. For instance, we have seen in Theorem 2, Statement 4, that, within a neighborhood of $\widehat{g}=0$, $\frac{\partial \widetilde{F}^{B}}{\partial \widehat{g}} \geq 0$. Its profile is similar to that of the third panel of Figure 1. That explains that the left-hand graph of the last row of Figure 6 is similar to the third panel of Figure $1\left(\gamma^{B}-\gamma^{A} \geq 0\right)$ and the right-hand graph of the same row is also similar but with the opposite $\operatorname{sign}\left(\gamma^{B}-\left(\phi \sigma_{s} \sigma_{f}+\gamma^{A}\right) \leq 0\right)$.
} 


\section{Figure 7: Constructing elementary contracts}
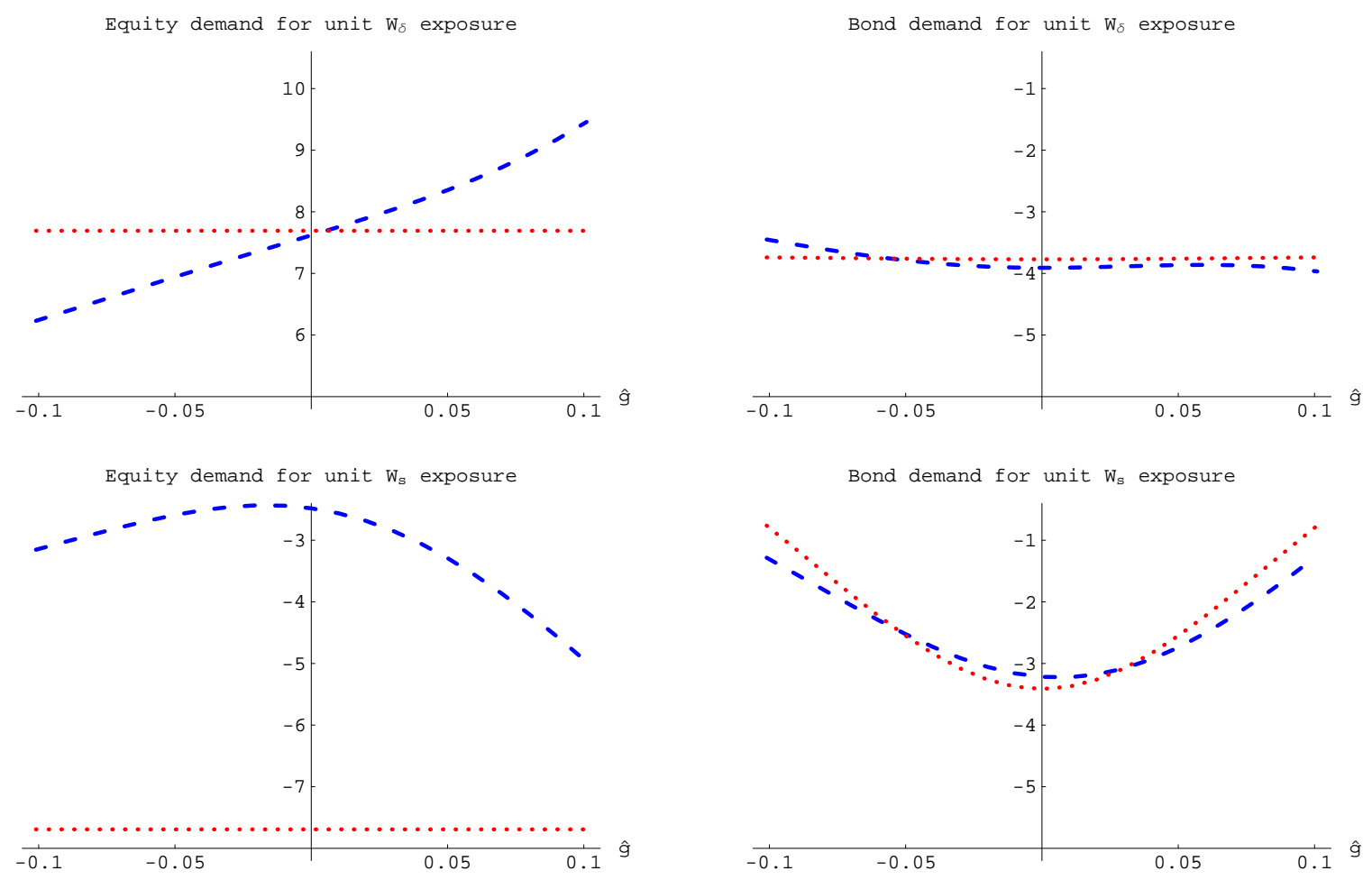

In each row of the figure, is plotted the composition of the portfolio of equity and bonds needed to construct a unit exposure to each of the two shocks. The four-cell figure is to be read row by row. For instance, the first row indicates that an investor who wishes to construct an exposure to the $d W_{\delta}$ shock amounting to $1 \%$ of his or her wealth should hold the equity in an amount equal to $7.8 \%$ of his or her wealth and short the bond in an amount equal to $3.77 \%$ of his or her wealth approximately. The parameter values and benchmark values used here are listed in Table 1 . In particular, average expectation, $f^{M}$, is held fixed at $\bar{f}$.

To understand the composition of the portfolio needed to construct a unit exposure to the fundamental shock, $d W_{\delta}$, and the signal shock, $d W_{s}$, note that equity is positively exposed to the innovation in the fundamental, $d W_{\delta}$. The reason for this is that a shock to the fundamental affects equity in two ways: it changes the payoff but also the valuation operator, and the positive effect dominates. ${ }^{47}$ On the other hand, the exposure of the bond to $d W_{\delta}$ is negative, because for bonds an innovation in the fundamental has only a valuation effect (it is harbinger of larger future output). For the same reason, both equity and bonds have negative exposures to innovations in the signal, $W_{s}$.

Because equity is positively exposed to the fundamental shock, the investor who wants to have positive exposure to the fundamental shock should hold equity positively. However, that would also expose him or her negatively to the signal shock. In order to construct a pure exposure to the fundamental shock and remove the unwanted negative exposure to the signal shock, one needs to hold negatively the bond which has a negative exposure to the signal shock. This is shown in the two plots that are displayed in the first row of Figure 7. On the other hand, the second row of Figure 7 shows that in order to construct a pure positive exposure to the signal shock, one would hold both equity and the bond negatively because each is negatively exposed to the signal shock. The ratio in which one holds them is designed to cancel off the exposure to the fundamental shock.

The plots in the first column of Figure 7 show that in the rational case (dotted line), the amount of equity needed as a percentage of $B$ 's wealth for a unit exposure to fundamental and signal shocks,

\footnotetext{
${ }^{47}$ This should not be confused with the negative effect of an increase in $\widehat{f}^{B}$ on the price of equity for the case where $\alpha<0$. In contrast to an increase in $\widehat{f}^{B}$, an increase in the fundamental, $\delta$, has an effect also on the other state variables.
} 
$d W_{\delta}$ and $d W_{s}$, are both equal to constants, the former being equal to $1 / \sigma_{\delta}$, and the latter to $-\sigma_{s} / \sigma_{\delta}^{2}$. Under irrationality, the amount of equity needed to generate a unit exposure to the fundamental shock rises with disagreement. This is because, in a neighborhood of $\widehat{g}=0$, the exposure of equity to the fundamental shock drops with $\widehat{g}$. It does so because its $\widehat{g}$-exposure components drop. ${ }^{48}$ The plots in the second column of Figure 7 show that the effect of irrationality on the bonds needed for a unit exposure to fundamental and signal shocks is small; that is, the difference between the dotted curves for rationality and the dashed curves for irrationality is small.

\subsection{Portfolio of Group $B$ in terms of equity, bond and riskless asset}

The equilibrium exposures demanded by Group B, which we identified in Subsection 6.2, can now be implemented by means of equity, the bond and the riskless asset, as explained in Subsection 6.3. This will give the portfolio strategy of Group $B$ investors. That is, premultiplying the vector of exposure demands to fundamental and signal shocks $d W_{\delta}$ and $d W_{s}$ (shown in the top row of Figure 6), by the matrix of equity and bonds demanded in order to generate a unit exposure to each of the two shocks (shown in Figure 7), gives the optimal portfolio weights allocated to equity and bonds. Below, we provide the economic intuition for these optimal portfolio weights.

Figure 8 gives Group B's portfolio holding (expressed as a percentage of wealth). In all the plots of this figure, the variable on the $x$-axis is the dispersion in beliefs, $\widehat{g}$. There are two columns of plots, with the one on the left giving the position in equity and the one on the right giving the position in bonds. The two plots in the first row give the overall investment in stocks and in bonds. The second row gives the static (mean-variance or myopic) investment in stocks and in bonds; the third row gives the investment in stocks and bonds that is done in order to hedge intertemporally against changes in all the state variables. ${ }^{49}$

Let us start with the case in which Group $A$ is rational ( $\phi=0$; dotted line) and agreement prevails $(\widehat{g}=0)$. Then, the two groups are identical and the top row of Figure 8 shows that in this case both groups are $100 \%$ invested in equity and $0 \%$ in bonds. We can also relate this result to the exposure demands that we have identified. Recall from Subsection 6.3 that holding only equity leads to a positive exposure to $W_{\delta}$ and a negative exposure to $W_{s}$, which is exactly the exposure desired by this investor (see the two plots in the first row of Figure 6).

Now, consider the case where there is some current disagreement $(\widehat{g} \neq 0)$ while retaining rationality (dotted line corresponding to $\phi=0$ ). Recall, from Subsection 6.1 and Figure 6, that the investor wishes to achieve a positive exposure to the fundamental shock, which rises with disagreement, and a negative exposure to signal shock, which also rises with disagreement. The first plot of Figure 8 shows that Group B continues to be $100 \%$ invested in equity irrespective of the disagreement but takes positions in the bond market that decline with a rise in $\widehat{g}$. The demand curve for bonds is approximately symmetric around the benchmark value of $\widehat{g}$. The holding of equity, as before, generates a positive exposure to $W_{\delta}$ and a negative exposure to $W_{s}$. On the other hand, shorting bonds leads to a positive exposure to $W_{\delta}$, and also a positive exposure to $W_{s}$. Thus, the short position in bonds, which increases in magnitude with $\widehat{g}$, leads to exactly the desired increase in the exposure to the two shocks, as shown in Figure 6.

Consider now the case of irrationality $(\phi>0)$. From the top row of Figure 8 , we see that the total bond holdings are virtually the same between the two cases of irrationality and rationality, and the main change is for equity (compare dashed and dotted lines). In the case of irrationality (dashed line) and agreement $(\widehat{g}=0)$, the rational Group $B$ agents invest into equity a smaller fraction of their wealth than they would in a rational market. The reason is that rational, risk averse investors are deterred by the presence of the irrational traders, who are a source of risk in their eyes. Hence, rational investors prefer to take refuge in the riskless short-term asset unless they are very optimistic about future growth.

\footnotetext{
${ }^{48}$ This fact can be traced back to Statement 4 of Lemma 2 and Theorem 2 and the nonincreasing lines of the third panel of Figure 1, themselves direct results of Jensen's inequality. That effect is shut out under rationality because $\widehat{g}$ then has zero diffusion.

${ }^{49}$ The intertemporal hedge itself could be broken down into three hedges against the three state variables: $\eta, \widehat{f}^{B}$ and $\widehat{g}$.
} 
Figure 8: Group B's portfolio weights for equity and bonds
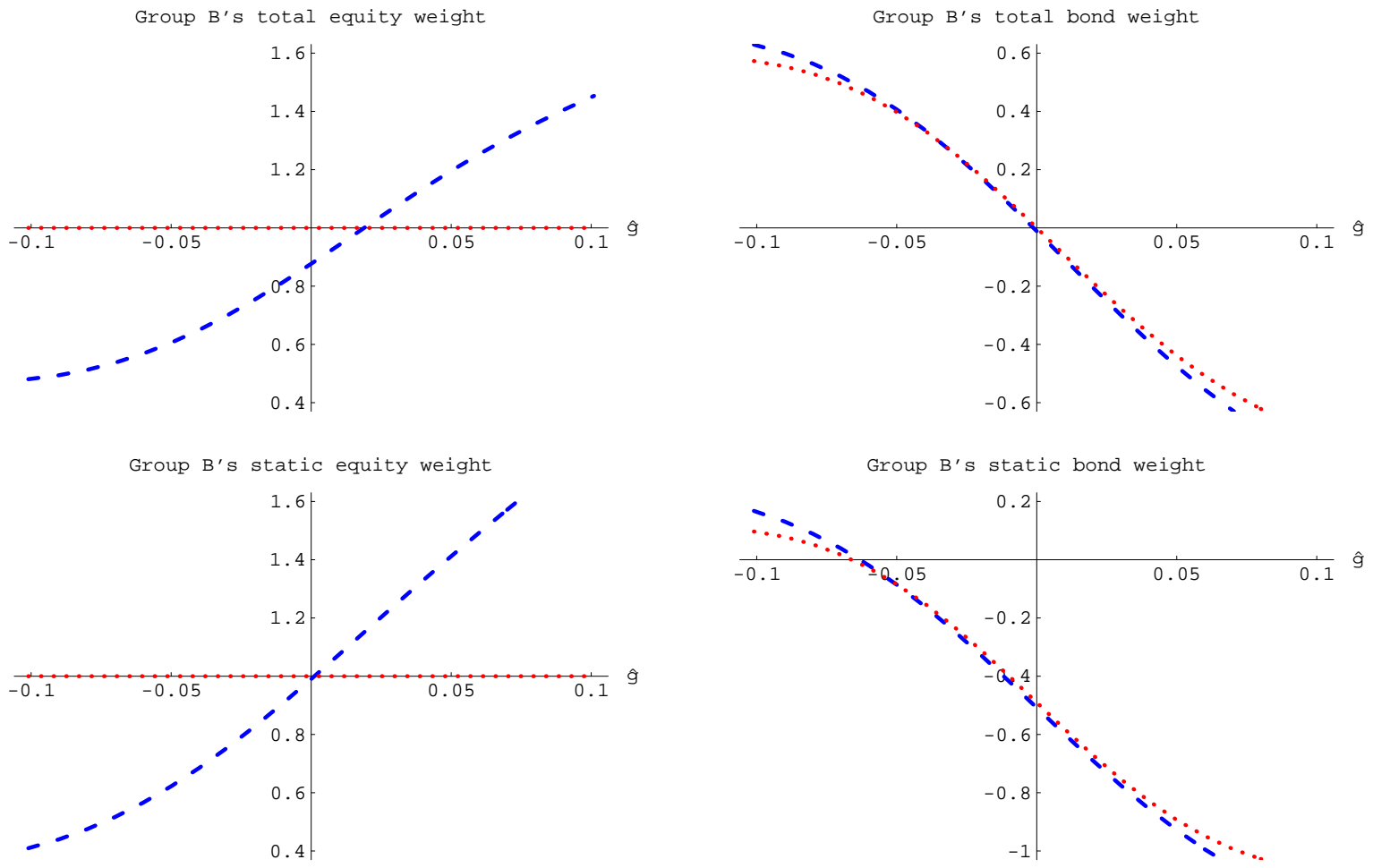

Group $B^{\prime}$ s hedging equity weight
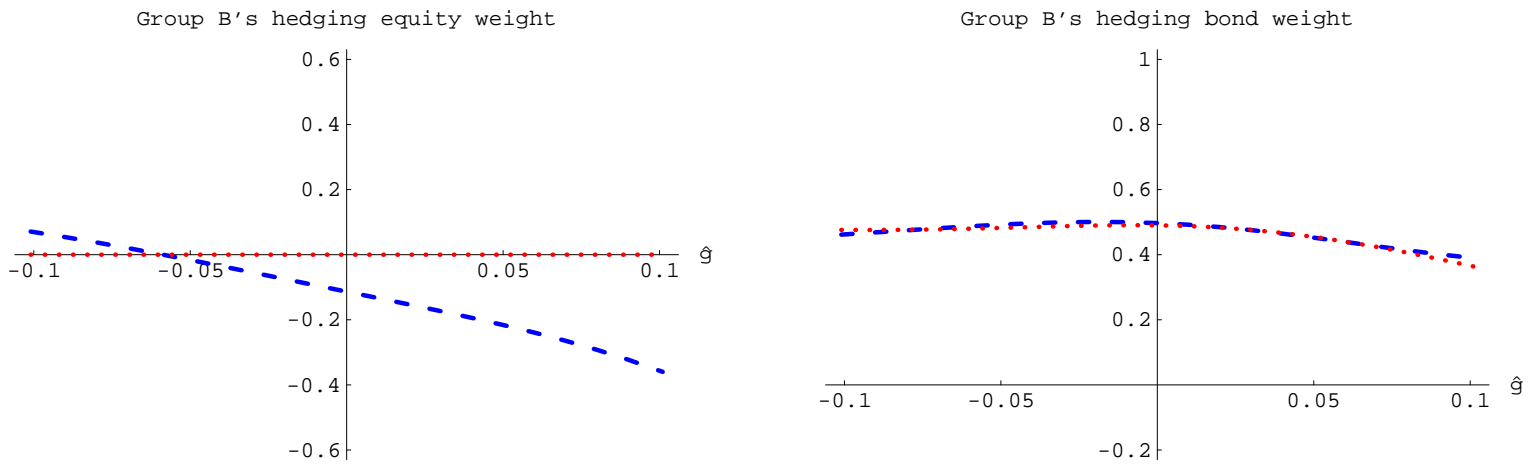

Group B's optimal portfolio weights are plotted in the first row, against $\widehat{g}$, for $\phi=0$ (dotted line) as well as $\phi=0.95$ (dashed curve). Rows 2 and 3 of the figure decompose the exposure demands into a static, short-term component (driven by current expected returns) and an intertemporal hedging component. The parameter values used here are given in Table 1. In particular, the two groups of investors have equal weights and average expectation, $f^{M}$, is held fixed at $\bar{f}$. 
As we did for the case of rationality, we can relate the total portfolio holdings to the optimal exposure demands under irrationality. Recall from the top row of Figure 6 that, in the case of irrationality, the positive exposure to the fundamental shock and the negative exposure to signal shock are both increased compared to the case of rationality and that this happens along parallel lines. How does this translate into equity and bond holdings? On the side of bonds, there is not a clear difference between the demands for them in the cases of rationality and irrationality (see the right-hand-side plot in the first row of Figure 8). While the weight allocated to bonds turns out not to change much, the weight allocated to equity does change substantially and in a non parallel fashion (see the left-hand-side plot in the first row of Figure 8). The reason for the change in the weight allocated to equity is to be found in Figure 7, which shows that there is a substantial change in the amount of equity needed to construct a unit exposure to the two shocks as one goes from the case of rationality to that of irrationality. As we saw, because of the $\widehat{g}$-exposure components of equity, this change is especially significant in the case of disagreement $(\widehat{g} \neq 0)$. Thus, in order to have about the same exposure under irrationality as under rationality, the optimal holding of equity has to change significantly.

To understand the motivations underlying the optimal portfolio of Group $B$ investors, we can decompose the total portfolio into a "static" component and an "intertemporal-hedging" component, as suggested in Merton $(1971,1973)$ and as was done above for exposure demands. Doing this for the bond (Figure 8, second column), we discover that the zero demand for the bond at the point $\widehat{g}=0$ under rationality ( $\phi=0$, dotted line) is the sum of a negative static component and a positive hedging component, each amounting to about half the size of the investor's wealth for our parameter configuration. The static component (plotted in the second row, right column) reflects beliefs about returns and the exploitation of return predictability. When Group $B$ is, for instance, optimistic about future growth $(\widehat{g}>0)$, it shorts the bond and invests the proceeds in the short-term deposit. This is because it expects the short rate to rise relative to the current value of long rates. Equity would be an inferior way to speculate about future expected growth, or about future spot interest rates, because the realized one-period output flow $\delta$ would introduce noise in ex post returns that is absent with bonds. Indeed, the static demand for equity is not very different from one.

The bond is a hedge, even in the absence of disagreement, for a reason that is the mirror image of the reason for which it is a speculative instrument in the presence of disagreement. Even when investors happen to agree about expected future growth today, they know that tomorrow both of their opinions will have evolved. The hedge operates as follows. If, tomorrow, Group B's belief $\widehat{f}^{B}$ rises to a higher level than today, bond prices are reduced so that a person holding a short position in the bond collects an offsetting return. The protection operates also in the case of a reduced belief of growth. Investors also use the bond as a hedge against future disagreement, $\widehat{g}=\widehat{f}^{B}-\widehat{f}^{A}$. Both hedges work in the same direction because we are currently looking at the demand of Group $B$. Given the high volatility of interest rates in our model, the hedging demand is also quite sizable - it represents around half of the wealth of Group $B$ in our numerical example. The strong hedging pressure brings down the expected excess return on bond, which explains why the static demand is strongly negative.

The motives behind the components of demand for bonds remain what they were under irrationality. The static demand reflects a desire to take a bet on future growth by means of term structure bets. The hedging demand remains strong at approximately the same level as under rationality.

Just like we did for bonds, we can also decompose the holding of equity into a static and an intertemporal hedging component. We have already discussed above that the aggregate holding of equity in the case of irrationality (dashed line) and agreement $(\widehat{g}=0)$ is a smaller fraction of wealth than it would be in a rational market because rational, risk averse investors are deterred by the presence of the irrational traders. Hence, rational investors prefer to take refuge in the riskless short-term asset unless they are extremely optimistic about future growth. The reduced equity position is a way to hedge fluctuations in the beliefs (see bottom row, first column of Figure 8).

If, however, the rational investors are currently more optimistic than the irrational ones $(\widehat{g}>0)$, they overcome their fear and invest in equities. The opposite is, of course, true when they are pessimistic. The demand for equity is mostly due to static demand (see first column, second row of Figure 8). 
However, because investors may change their minds in subsequent periods, the increased static demand in case of optimism is tempered by an accompanying increased hedging demand for stocks (first column, third row of Figure 8). It is at first puzzling that the static demand for equity seems greatly affected by irrationality. Since this is the static component, it would appear that investors do this in order to exploit predictability in stock returns. When examining exposure demands, however, we concluded that the static exposure demand was left unchanged by irrationality so that: "Exploiting the return predictability created by irrationality is not a concern of Group B's portfolio strategy." The explanation of this apparent contradiction is the same as the one we gave above for the fact that the presence of irrationality greatly affects equity demand in general: there is a substantial change in the amount of equity needed to construct a unit exposure to the two shocks as one goes from the case of rationality to that of irrationality. As we saw, however, this was related to the $\widehat{g}$-exposure components of equity, not to the static components of equity.

Summarizing the optimal portfolio choice of the rational Group B:

Proposition 5 In the case of irrationality as in the case of rationality, the bond serves both to take a view on future expected growth rates and to hedge against their revisions which will be reflected in future bond prices. The total holding of equity in the case of irrationality is reduced relative to the case of rationality when there is agreement, because risk averse investors are deterred by the presence of the irrational traders. Under disagreement, which drives predictability, the position of rational investors in equity is strongly influenced by whether they are currently more optimistic or pessimistic than irrational investors. However, because investors may change their minds in subsequent periods, the increased static demand for equity in case of optimism is tempered by an increased, negative hedging demand for stocks.

In conclusion, we have exhibited the way in which rational risk-arbitrageurs trade on their beliefs that the market is being foolish. When doing so, they must hedge future fluctuations in the market's sentiment. This illustrates the general idea that risk arbitrage cannot just be based on a current price divergence. It must also be based on a model of irrational behavior and a prediction concerning the speed of convergence. The risk arbitrage must include a protection in case there is a deviation from that prediction, a form of risk that David (2004) has called "trading risk" and that we called "sentiment risk". We have found that, for the current form of heterogeneous beliefs, bonds are an essential accompaniment of equity investment, in order to hedge the trading or sentiment risk that is present in the financial market.

\section{$7 \quad$ Profits of rational investors $v s$. survival of overconfident agents}

We now return to the question we asked originally concerning the potential for gains that the excessive volatility creates for the rational investors who follow the portfolio strategy that we described in the previous section. By asking whether rational risk arbitrageurs can take advantage of overconfident investors, we simultaneously ask whether rational investors eliminate irrational investors from the economy very quickly, or whether overconfident investors can survive for some time.

The survival of irrational traders is an issue that was raised by De Long, Shleifer, Summers, and Waldmann (1990a, 1991) in a partial-equilibrium setting, in which traders did not affect prices. The survival of excessively optimistic or pessimistic agents, in an economy in which one category of agents knows the true probability distribution, is the focus of recent papers by Kogan, Ross, Wang, and Westerfield (2003) and Yan (2004). Here, however, we consider a different kind of irrational agents, who change their mind too frequently, being sometimes too optimistic and at other times too pessimistic about the growth rate of aggregate dividends, as compared to the beliefs of Bayesian investors, not as compared to the truth. Kogan, Ross, Wang, and Westerfield (2003) considers agents who consume only at some terminal horizon date so that their saving rate is not optimized and the growth of the economy is not an important factor in the analysis. Yan (2004), in contrast, considers agents who consume intertemporally and make optimal savings decisions. In an economy the specification of which is very close to ours, Berrada (2004) has discussed the issue of survival by means of simulations. 
Figure 9: Survival of the irrational Group $A$
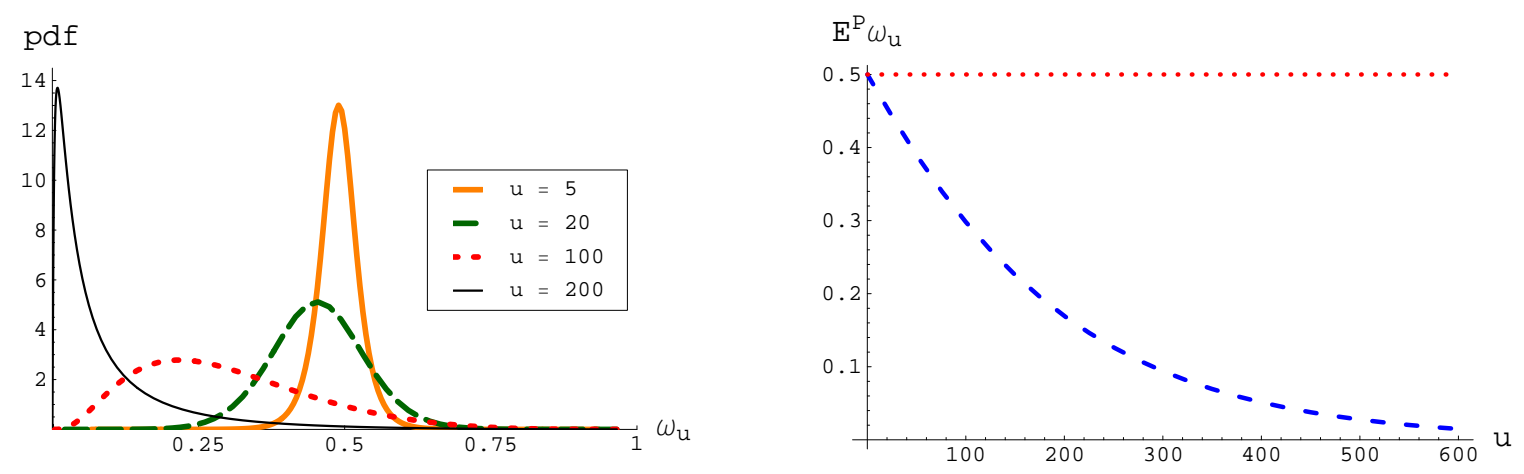

The plot on the left gives the probability density function (pdf) of Group $A$ 's share of consumption, $\omega_{u}$, after the passage of $u$ years. The plot on the right shows the expected value under the objective measure of Group $A$ 's consumption share, $\mathbb{E}_{0}^{P} \omega_{u}$, as a function of time measured in years, with current time assumed to be 0 and future time denoted by $u$ on the $x$-axis. In the second plot, the dotted line represents the case where $\phi=0$ and all agents are rational, while the dashed line represents the case where $\phi=0.95$ implying that Group $A$ is irrational. The parameter values used here are given in Table 1. In particular, the two groups of investors have equal initial weights, $\omega_{0}=1 / 2$.

Most previous studies, with the exception of Berrada (2004), have defined "survival" in terms of the irrational agents' asymptotic share of wealth as the horizon goes to infinity. In general, however, wealth is a sufficient summary statistic neither of an agent's welfare nor of his or her influence on asset prices. Under von Neuman-Morgenstern, time-additive utility, the share of consumption is such a summary statistic. Therefore, we measure the survival of irrational agents in the economy by studying the evolution of the share of total dividend that will be consumed by them. The probability distribution of this share is computed under the objective, or true, probability measure rather than under the measure of either Group $A$ or $B$.

Group A's ratio of consumption to aggregate dividends is given by Equation (35). To compute the distribution of this ratio, we need the conditional distribution of $\eta_{u}$, given $\eta_{t}, f_{t}, \widehat{f}_{t}^{A}, \widehat{f}_{t}^{B}$ at $t$. As in Subsection 3.3, we first obtain its characteristic function (see Lemma 3 in Appendix A). Then, the expected value and the probability distribution of the share of Group A's consumption in (35) can be obtained by means of Fourier inversion (as shown in Equation (B4) of Appendix B).

We study the case where Groups $A$ and $B$ start out with the same estimate of the future growth rate, that is, $\widehat{f}^{A}=\widehat{f}^{B}$ and also consume the same share of aggregate dividend, $\omega_{0}=1 / 2$. Recall also that we are assuming that both categories of agents have the same, time additive, isoelastic utility function. In the left panel of Figure 9, we plot the probability density function of Group $A$ 's share of consumption after the elapse of different numbers of years. We see from the left panel that as time passes, the density moves to the left, and thus, Group A's share of consumption is decreasing. To understand the rate at which this share is decreasing, in the right panel we plot the expected value of this share, $\mathbb{E}_{0}^{P} \omega_{u}$, against time measured in years. This plot in the right panel considers two cases: one, where Group $A$ is Bayesian $(\phi=0)$ and the other where Group $A$ is irrational $(\phi=0.95)$.

Both plots confirm that ultimately, irrational agents become extinct in that their share of consumption vanishes. But, the more interesting observation is that, in contrast to what is typically assumed in models of rational asset pricing, irrational agents do not lose out right away. For instance, the right-hand side plot in Figure 9 shows that after 100 years the overconfident agents' expected share of consumption of the aggregate dividends has dropped only to $30 \%$ from the initial share of $50 \%$. Recall that in Figure 4 we showed that the relation between the volatility of equity returns and the consumption share of Group $A$ is linear. The fact that the irrational group is not eliminated from the population instantly implies that the phenomenon of excess volatility will also not be eliminated quickly. 


\section{Conclusion}

In a capital market characterized by excessive volatility, we have analyzed the price and return behavior that would prevail in equilibrium and the trading strategy that would allow a rational investor to take advantage of the excess volatility. To achieve our goal, we constructed a general-equilibrium model in which stock prices were excessively volatile, using the device proposed by Scheinkman and Xiong (2003). In our model, there are two groups of agents, and one group (irrational or overconfident) believes that the magnitude of the correlation between the innovations in the signal and innovations in some unobserved variable (the expected growth rate of dividends) is larger than it actually is. Consequently, when a signal is received, this group of agents adjusts their beliefs too much and overreacts to it, which then generates excessive stock price movements. The excess movement was regarded as a "sentiment" factor.

For given beliefs, however, both classes of agents are rational in their decision making, in the sense that both are intertemporal optimizers. In this way, the irrational investors are not sitting ducks. Rational investors have to engage in a fairly intricate investment strategy to triumph over the irrational ones. And their victory can only be achieved in the fairly long run.

We believe that this undertaking brings two benefits. First, given that we have worked out in careful detail the optimal portfolio strategies to be followed by rational people and defined the derivative securities that would facilitate the implementation of these strategies, our model should be of practical use to hedge funds who play the price-convergence game. They often have at their disposal perfectmarket pricing models that allow them to spot pricing anomalies. But, that is not sufficient information to be able to put in place a "risk arbitrage" strategy, including the optimal timing of trades into the strategy, of trades out of the strategy, plus the accompanying hedges. For that purpose, hedge funds also need a model of the equilibrium stochastic process of price spreads. We provide one such model. We caution, however, that the gains will accumulate at a slow pace.

Second, the model combines the technical virtues of continuous-time, rational-expectations equilibrium asset pricing models (including the use of the martingale approach) with a single, well-defined, almost axiomatic deviation from rational Bayesian learning. In this way, it has allowed us to analyze the consequences for asset prices and portfolio strategies of that specific deviation. We hope that this model, or similar models obtained by this method, can become workhorses in the development of behavioral equilibrium theory. 


\section{A The moment generating functions}

Lemma 1 The moment-generating-function for the joint distribution of $\delta$ and $\eta$ at maturity $u$ under the measure of Group B is given by

$$
\mathbb{E}_{\widehat{f}^{B}, \widehat{g}}^{B}\left[\left(\frac{\delta_{u}}{\delta}\right)^{\varepsilon}\left(\frac{\eta_{u}}{\eta}\right)^{\chi}\right]=H_{f}\left(\widehat{f}^{B}, t, u ; \varepsilon\right) \times H_{g}(\widehat{g}, t, u ; \varepsilon, \chi),
$$

with:

$$
\begin{aligned}
H_{f}\left(\widehat{f}^{B}, t ; u, \varepsilon\right)= & \mathbb{E}_{\widehat{f}^{B}}^{B}\left[\left(\frac{\delta_{u}}{\delta}\right)^{\varepsilon}\right] \\
= & \exp \left\{\varepsilon\left[\bar{f}(u-t)+\frac{1}{\zeta}\left(\widehat{f}^{B}-\bar{f}\right)\left[1-e^{-\zeta(u-t)}\right]\right]+\frac{1}{2} \varepsilon(\varepsilon-1) \sigma_{\delta}^{2}(u-t)\right. \\
& \left.+\frac{\varepsilon^{2} \gamma^{B}}{2 \zeta^{2}}\left[1-e^{-\zeta(u-t)}\right]^{2}+\frac{\varepsilon^{2} \sigma_{f}^{2}}{4 \zeta^{3}}\left[2 \zeta(u-t)-3+4 e^{-\zeta(u-t)}-e^{-2 \zeta(u-t)}\right]\right\},
\end{aligned}
$$

and

$$
H_{g}(\widehat{g}, t, u ; \varepsilon, \chi)=\exp \left\{A_{1}(\chi ; u-t)+\varepsilon^{2} A_{2}(\chi ; u-t)+\varepsilon \widehat{g} B(\chi ; u-t)+\widehat{g}^{2} C(\chi ; u-t)\right\},
$$

where the functions $A_{1}(\chi ; u-t), A_{2}(\chi ; u-t), B(\chi ; u-t)$, and $C(\chi ; u-t)$ are given explicitly in the proof. Moreover, the moment-generating-function is finite and real for $0 \leq \chi \leq 1$ and $\varepsilon \in \mathbb{R}$.

Proof: We want to compute

$$
H\left(\delta, \eta, \widehat{f}^{B}, \widehat{g}, t, u ; \varepsilon, \chi\right)=\mathbb{E}_{\delta, \eta, \widehat{f}^{B}, \widehat{g}}^{B}\left[\left(\delta_{u}\right)^{\varepsilon}\left(\eta_{u}\right)^{\chi}\right] .
$$

This function satisfies the linear PDE:

$$
0 \equiv \mathcal{D} H\left(\delta, \eta, \widehat{f}^{B}, \widehat{g}, t, u ; \varepsilon, \chi\right)+\frac{\partial H}{\partial t}\left(\delta, \eta, \widehat{f}^{B}, \widehat{g}, t, u ; \varepsilon, \chi\right),
$$

with the initial condition $H\left(\delta, \eta, \widehat{f}^{B}, \widehat{g}, t, t ; \varepsilon, \chi\right)=\delta^{\varepsilon} \eta^{\chi}$, and where $\mathcal{D}$ is the differential generator of $\left(\delta_{t}, \eta_{t}, \widehat{f}_{t}^{B}, \widehat{g}_{t}\right)$ under the probability measure of Group $B$.

Spelling out (A6) we have:

$$
\begin{aligned}
0= & \frac{\partial H}{\partial \delta} \delta \widehat{f}^{B}-\frac{\partial H}{\partial \widehat{f}^{B}} \zeta\left(\widehat{f}^{B}-\bar{f}\right)-\frac{\partial H}{\partial \widehat{g}} \widehat{g}\left(\zeta+\frac{\phi \sigma_{s} \sigma_{f}+\gamma^{A}}{\sigma_{s}^{2}}+\frac{\gamma^{A}}{\sigma_{\delta}^{2}}\right)+\frac{1}{2} \frac{\partial^{2} H}{\partial \delta^{2}}\left(\delta \sigma_{\delta}\right)^{2} \\
& +\frac{1}{2} \frac{\partial^{2} H}{\partial \eta^{2}}(\eta \widehat{g})^{2}\left(\frac{1}{\sigma_{\delta}^{2}}+\frac{1}{\sigma_{s}^{2}}\right)+\frac{1}{2} \frac{\partial^{2} H}{\partial \widehat{g}^{2}}\left[\left(\frac{\gamma^{B}-\gamma^{A}}{\sigma_{\delta}}\right)^{2}+\left(\frac{\gamma^{B}-\left(\phi \sigma_{s} \sigma_{f}+\gamma^{A}\right)}{\sigma_{s}}\right)^{2}\right] \\
& +\frac{1}{2} \frac{\partial^{2} H}{\partial\left(\widehat{f}^{B}\right)^{2}}\left(\frac{1}{\sigma_{\delta}^{2}}+\frac{1}{\sigma_{s}^{2}}\right)\left(\gamma^{B}\right)^{2}-\frac{\partial^{2} H}{\partial \delta \partial \eta} \delta \eta \widehat{g}+\frac{\partial^{2} H}{\partial \delta \partial \widehat{g}} \delta\left(\gamma^{B}-\gamma^{A}\right) \\
& +\frac{\partial^{2} H}{\partial \delta \partial \widehat{f}^{B}} \delta \gamma^{B}-\frac{\partial^{2} H}{\partial \eta \partial \widehat{g}} \eta \widehat{g}\left(\frac{\gamma^{B}-\gamma^{A}}{\sigma_{\delta}^{2}}+\frac{\gamma^{B}-\left(\phi \sigma_{s} \sigma_{f}+\gamma^{A}\right)}{\sigma_{s}^{2}}\right) \\
& -\frac{\partial^{2} H}{\partial \eta \partial \widehat{f}^{B}} \eta \widehat{g}\left(\frac{1}{\sigma_{\delta}^{2}}+\frac{1}{\sigma_{s}^{2}}\right) \gamma^{B}+\frac{\partial^{2} H}{\partial \widehat{g} \partial \widehat{f}^{B}}\left(\frac{\gamma^{B}-\gamma^{A}}{\sigma_{\delta}^{2}}+\frac{\gamma^{B}-\left(\phi \sigma_{s} \sigma_{f}+\gamma^{A}\right)}{\sigma_{s}^{2}}\right) \gamma^{B}+\frac{\partial H}{\partial t} .
\end{aligned}
$$

The solution of this PDE is

$$
H\left(\delta, \eta, \widehat{f}^{B}, \widehat{g}, t, u ; \varepsilon, \chi\right)=\delta^{\varepsilon} \eta^{\chi} \times H_{f}\left(\widehat{f}^{B}, t, u ; \varepsilon\right) \times H_{g}(\widehat{g}, t, u ; \varepsilon, \chi),
$$


where $H_{f}\left(\widehat{f}^{B}, t, u ; \varepsilon\right)$ and $H_{g}(\widehat{g}, t, u ; \varepsilon, \chi)$ are defined in (A3) and (A4). ${ }^{50}$ Substituting (A8) into the $\mathrm{PDE}$ and simplifying, we find that the functions of time, $A_{1}, A_{2}, B$ and $C$, that are present in (A4) need to solve the following ODEs:

$$
\begin{aligned}
C^{\prime}(u-t)= & a C^{2}(u-t)-2 b C(u-t)+c, \quad C(0)=0 \\
B^{\prime}(u-t)= & B(u-t)[a C(u-t)-b]+k+l e^{-\zeta(u-t)} \\
& +2\left[m+n e^{-\zeta(u-t)}\right] C(u-t), \quad B(0)=0 \\
A_{1}^{\prime}(u-t)= & \frac{a}{2} C(u-t), \quad A_{1}(0)=0, \\
A_{2}^{\prime}(u-t)= & B(u-t)\left[m+n e^{-\zeta(u-t)}+\frac{a}{4} B(u-t)\right], \quad A_{2}(0)=0
\end{aligned}
$$

where

$$
\begin{aligned}
& a=2\left[\left(\frac{\gamma^{B}-\gamma^{A}}{\sigma_{\delta}}\right)^{2}+\left(\frac{\gamma^{B}-\left(\phi \sigma_{s} \sigma_{f}+\gamma^{A}\right)}{\sigma_{s}}\right)^{2}\right] \\
& b=\zeta+\frac{\phi \sigma_{s} \sigma_{f}+\gamma^{A}}{\sigma_{s}^{2}}+\frac{\gamma^{A}}{\sigma_{\delta}^{2}}+\chi\left(\frac{\gamma^{B}-\gamma^{A}}{\sigma_{\delta}^{2}}+\frac{\gamma^{B}-\left(\phi \sigma_{s} \sigma_{f}+\gamma^{A}\right)}{\sigma_{s}^{2}}\right), \\
& c=\frac{1}{2} \chi(\chi-1)\left(\frac{1}{\sigma_{\delta}^{2}}+\frac{1}{\sigma_{s}^{2}}\right),
\end{aligned}
$$

and

$$
\begin{aligned}
k & =-\chi\left[1+\frac{\gamma^{B}}{\zeta}\left(\frac{1}{\sigma_{\delta}^{2}}+\frac{1}{\sigma_{s}^{2}}\right)\right] \\
l & =\chi \frac{\gamma^{B}}{\zeta}\left(\frac{1}{\sigma_{\delta}^{2}}+\frac{1}{\sigma_{s}^{2}}\right) \\
m & =\gamma^{B}-\gamma^{A}+\frac{\gamma^{B}}{\zeta}\left(\frac{\gamma^{B}-\gamma^{A}}{\sigma_{\delta}^{2}}+\frac{\gamma^{B}-\left(\phi \sigma_{s} \sigma_{f}+\gamma^{A}\right)}{\sigma_{s}^{2}}\right) \\
n & =-\frac{\gamma^{B}}{\zeta}\left(\frac{\gamma^{B}-\gamma^{A}}{\sigma_{\delta}^{2}}+\frac{\gamma^{B}-\left(\phi \sigma_{s} \sigma_{f}+\gamma^{A}\right)}{\sigma_{s}^{2}}\right) .
\end{aligned}
$$

Denoting

$$
q=\sqrt{b^{2}-a c}
$$

and

$$
\begin{array}{ll}
v_{1}=0, & \vartheta_{1}=\frac{2 c m+k(b+q)}{q}, \\
v_{2}=2 q, & \vartheta_{2}=\frac{2 c m+k(b-q)}{q}, \\
v_{3}=\zeta, & \vartheta_{3}=\frac{2 c n+l(b+q)}{q-\zeta}, \\
v_{4}=2 q+\zeta, & \vartheta_{4}=\frac{2 c n+l(b-q)}{q+\zeta}, \\
v_{5}=q, & \vartheta_{5}=-\left(\vartheta_{1}+\vartheta_{2}+\vartheta_{3}+\vartheta_{4}\right),
\end{array}
$$

\footnotetext{
${ }^{50}$ To see that $H_{f}$, which is defined in (A2), is the moment generating function for $\delta_{u} / \delta$ under Group $B$ 's measure, one can verify that $\delta^{\varepsilon} H_{f}\left(\widehat{f}^{B}, t ; u, \varepsilon\right)$ solves the PDE in (A7) but with all terms that are the partial derivatives of $H$ with respect to either $\eta$ or $\widehat{g}$ dropped.
} 
we obtain

$$
\begin{aligned}
C(u-t) & =\frac{c\left(1-e^{-2 q(u-t)}\right)}{q+b+(q-b) e^{-2 q(u-t)}} \\
B(u-t) & =\frac{\sum_{i=1}^{5} \vartheta_{i} e^{-v_{i}(u-t)}}{q+b+(q-b) e^{-2 q(u-t)}} \\
A_{1}(u-t) & =\frac{a}{2} \int_{t}^{u} C(\tau-t) d \tau \\
& =\frac{1}{2}\left[(b-q)(u-t)+\ln (2 q)-\ln \left(q+b+(q-b) e^{-2 q(u-t)}\right)\right] \\
A_{2}(u-t) & =\int_{t}^{u} B(\tau-t)\left[m+n e^{-\zeta(\tau-t)}+\frac{a}{4} B(\tau-t)\right] d \tau \\
& =\sum_{j=1}^{5} \vartheta_{j}\left[m D_{1}\left(\vartheta_{j} ; u-t\right)+n D_{1}\left(\vartheta_{j}+\zeta ; u-t\right)\right]+\frac{a}{4} \sum_{i, j=1}^{5} \vartheta_{i} \vartheta_{j} D_{2}\left(\vartheta_{i}+\vartheta_{j} ; u-t\right)(
\end{aligned}
$$

where, denoting by $\mathcal{H}$ the standard hypergeometric function,

$$
\begin{aligned}
D_{1}(p ; u-t)=\int_{t}^{u} \frac{e^{-p(\tau-t)}}{q+b+(q-b) e^{-2 q(u-t)}} d \tau \\
\quad=\left\{\begin{array}{lr}
\frac{2 q(u-t)-\ln (2 q)+\ln \left(q+b+(q-b) e^{-2 q(u-t)}\right)}{2 q(q+b)}, & p=0, \\
\frac{1}{p(q+b)}\left[\mathcal{H}\left(1, \frac{p}{2 q}, 1+\frac{p}{2 q},-\frac{q-b}{q+b}\right)-e^{-p(u-t)} \mathcal{H}\left(1, \frac{p}{2 q}, 1+\frac{p}{2 q},-\frac{q-b}{q+b} e^{-2 q(u-t)}\right)\right], & p>0,
\end{array}\right.
\end{aligned}
$$

and

$$
\begin{aligned}
D_{2}(p ; u-t) & =\int_{t}^{u} \frac{e^{-p(\tau-t)}}{\left[q+b+(q-b) e^{-2 q(u-t)}\right]^{2}} d \tau \\
& =\frac{1}{2 q(q+b)}\left[\frac{1}{2 q}-\frac{e^{-p(u-t)}}{q+b+(q-b) e^{-2 q(u-t)}}+(2 q-p) D_{1}(p ; u-t)\right]
\end{aligned}
$$

To show that the function $H_{g}(\widehat{g}, t, u ; \varepsilon, \chi)$ is well defined for $\chi \in[0,1]$ and $u \geq t$, first note that the radicand in the expression (A20) for $q$ may be written as a quadratic trinomial of $\chi$ :

$$
b^{2}-a c=q_{2} \chi^{2}+q_{1} \chi+q_{0},
$$

where

$$
\begin{aligned}
& q_{2}=-\frac{\phi^{2} \sigma_{f}^{2}}{\sigma_{\delta}^{2}} \\
& q_{1}=2 \phi \sigma_{f}\left(\frac{\phi \sigma_{f}}{\sigma_{\delta}^{2}}-\frac{\zeta}{\sigma_{s}}\right), \\
& q_{0}=\left(\zeta+\frac{\phi \sigma_{f}}{\sigma_{s}}\right)^{2}+\left(1-\phi^{2}\right)\left(\frac{\sigma_{f}^{2}}{\sigma_{s}^{2}}+\frac{\sigma_{f}^{2}}{\sigma_{\delta}^{2}}\right) .
\end{aligned}
$$

Since $\phi \in[0,1]$ and $\zeta>0$, it is immediate that $q_{2} \leq 0, q_{0}>\zeta^{2}$, and

$$
q_{2}+q_{1}+q_{0}=\zeta^{2}+\frac{\sigma_{f}^{2}}{\sigma_{\delta}^{2}}+\frac{\sigma_{f}^{2}}{\sigma_{s}^{2}}>\zeta^{2} .
$$

So, when $\chi \in[0,1], q=\sqrt{b^{2}-a c}$ is real. Moreover, $q>\zeta$ so that $\left\{\vartheta_{i}\right\}_{i=1}^{5}$ are finite. 
Taking into account that $a \geq 0$, and for $\chi \in[0,1], c \leq 0$ and

$$
b=\chi \sqrt{\zeta^{2}+\frac{\sigma_{f}^{2}}{\sigma_{\delta}^{2}}+\frac{\sigma_{f}^{2}}{\sigma_{s}^{2}}}+(1-\chi) \sqrt{\left(\zeta+\frac{\phi \sigma_{f}}{\sigma_{s}}\right)^{2}+\left(1-\phi^{2}\right)\left(\frac{\sigma_{f}^{2}}{\sigma_{s}^{2}}+\frac{\sigma_{f}^{2}}{\sigma_{\delta}^{2}}\right)}>\zeta,
$$

we obtain that $q-b \geq 0$ and

$$
q+b+(q-b) e^{-2 q(u-t)} \geq q+b>0 .
$$

Therefore, when $\chi \in[0,1]$ and $u \geq t$, the functions $C(u-t)$ and $B(u-t)$ are well defined and bounded; the integrals $A_{1}(u-t)$ and $A_{2}(u-t)$ are convergent, and thus, their closed-form expressions (A29) and (A12) are also well defined.

\section{Proof of Lemma 2}

To prove Statement 1 , note that because $\eta_{u}$ is a martingale under the measure of Group $B$, for $0 \leq \chi \leq 1$, it must be the case by Jensen's inequality that $\mathbb{E}_{\widehat{g}}^{B}\left[\left(\frac{\eta_{u}}{\eta}\right)^{\chi}\right] \leq 1$ for any value of $\widehat{g}$. Because from (A4)

$$
\mathbb{E}_{\widehat{g}}^{B}\left[\left(\frac{\eta_{u}}{\eta}\right)^{\chi}\right]=H_{g}(\widehat{g}, t, u ; 0, \chi)=\exp \left\{A_{1}(\chi ; u-t)+\widehat{g}^{2} C(\chi ; u-t)\right\},
$$

this implies $A_{1}(\chi ; u-t) \leq 0$ and $C(\chi ; u-t) \leq 0$ and proves Statement 1 of the lemma.

To prove Statement 2, we need to show that in the expression

$$
\frac{H_{g}(\widehat{g}, t, u ; \varepsilon, \chi)}{H_{g}(\widehat{g}, t, u ; 0, \chi)}=\exp \left\{\varepsilon^{2} A_{2}(\chi ; u-t)+\varepsilon \widehat{g} B(\chi ; u-t)\right\},
$$

the functions $B(\chi ; u-t)$ and $A_{2}(\chi ; u-t)$ are non-positive. We prove this by contradiction. Assume the function $B(u-t)$ takes positive values. Then since this function is smooth and $B(0)=0$, there must exist $u_{1} \geq t$ such that $B\left(u_{1}-t\right)=0$ and $B^{\prime}\left(u_{1}-t\right)>0$. However, it can be shown that the RHS in (A10) is nonpositive at $u_{1}$. Actually, since $B\left(u_{1}-t\right)=0$ the first term in (A10) is equal to zero. Then from (A16) and (A17), $k, k+l \leq 0$ and hence $k+l e^{-\zeta\left(u_{1}-t\right)} \leq 0$. Finally, $m+n e^{-\zeta\left(u_{1}-t\right)} \geq 0$ since from (A18) and (A19)

$$
\begin{aligned}
m+n & =\gamma^{B}-\gamma^{A} \geq 0 \\
m & \equiv \frac{a}{4 \zeta} \geq 0
\end{aligned}
$$

where the last identity can be verified by substituting the expressions for $\gamma^{A}$ and $\gamma^{B}$ into (A13) and (A18). Therefore, by contradiction, we have shown that $B(u-t) \leq 0$.

To prove $A_{2}(u-t) \leq 0$, it is sufficient to show that in (A31)

$$
\Psi(u-t)=m+n e^{-\zeta(u-t)}+\frac{a}{4} B(u-t) \geq 0 .
$$

Assume that $\Psi(u-t)$ is negative. Then since $\Psi(u-t)$ is smooth and $\Psi(0)=m+n \geq 0$, there exists $u_{2} \geq t$ such that $\Psi\left(u_{2}-t\right)=0$ and $\Psi^{\prime}\left(u_{2}-t\right)<0$. On the other hand, substituting $\frac{a}{4} B\left(u_{2}-t\right)=$ $-m-n e^{-\zeta\left(u_{2}-t\right)}$ into (A10) and taking into account (A44) we have

$$
\begin{aligned}
\Psi^{\prime}\left(u_{2}-t\right) & =-\zeta n e^{-\zeta\left(u_{2}-t\right)}+\frac{a}{4} B^{\prime}\left(u_{2}-t\right) \\
& =-\zeta n e^{-\zeta\left(u_{2}-t\right)}-\left(m+n e^{-\zeta\left(u_{2}-t\right)}\right)\left[2 \zeta m C\left(u_{2}-t\right)-b\right]+\zeta m\left[k+l e^{-\zeta\left(u_{2}-t\right)}\right] \\
& \geq m(b+\zeta k)+(b n+\zeta m l-\zeta n) e^{-\zeta\left(u_{2}-t\right)} \geq 0,
\end{aligned}
$$


where to get the last inequality we find for $\chi \in[0,1]$ :

$$
b+\zeta k=(1-\chi) \sqrt{\left(\zeta+\frac{\phi \sigma_{f}}{\sigma_{s}}\right)^{2}+\left(1-\phi^{2}\right)\left(\frac{\sigma_{f}^{2}}{\sigma_{s}^{2}}+\frac{\sigma_{f}^{2}}{\sigma_{\delta}^{2}}\right)} \geq 0,
$$

and taking into account (A39):

$$
\begin{aligned}
m(b+\zeta k)+(b n+\zeta m l-\zeta n) & =b(m+n)-\zeta(\chi m+n) \\
& \geq(b-\zeta)(m+n) \geq 0 .
\end{aligned}
$$

So, $\Psi(u-t) \geq 0$ and $A_{2}(u-t) \leq 0$. This completes the proof of Statement 2 of the lemma.

To prove Statement 3 first note that, when $\phi=0, a=m=n=0$ (see Equations (A13), (A18) and (A19)) and the functions $A_{1}(\chi ; u-t)$ and $A_{2}(\chi ; u-t)$, as defined through (A28) and (A30), are zero. Therefore, when $\phi=0$ and $\widehat{g}=0, H_{g}(\widehat{g}, t, u ; \varepsilon, \chi)=1$. On the other hand, when $\phi>0$ and also $u>t$ and $0<\chi<1, a>0$, and from (A26) the function $C(\chi ; u-t)$ is not zero and, thus, strictly negative. Consequently, from (A28) $A_{1}(\chi ; u-t)<0$ and, if in addition $\widehat{g}=0$ or $\varepsilon=0$, then $H_{g}(\widehat{g}, t, u ; \varepsilon, \chi)<1$.

Statement 4 follows directly from

$$
\frac{1}{H_{g}} \frac{\partial H_{g}}{\partial \widehat{g}}(\widehat{g}, t, u ; \varepsilon, \chi)=\varepsilon B(\chi ; u-t)+2 \widehat{g} C(\chi ; u-t),
$$

and the previously established results that $B(\chi ; u-t) \leq 0$ and $C(\chi ; u-t) \leq 0$.

Finally, from (A3)

$$
\frac{1}{H_{f}} \frac{\partial H_{f}}{\partial \widehat{f}^{B}}\left(\widehat{f}^{B}, t, u ; \varepsilon\right)=\frac{\varepsilon}{\zeta}\left(1-e^{-\zeta(u-t)}\right),
$$

which verifies Statement 5 and completes the proof of the lemma.

Lemma 3 The moment generating function of $\eta$ under the objective probability measure is

$$
\mathbb{E}_{\widehat{g}^{A}, \widehat{g}^{B}}^{P}\left[\frac{\eta_{u}}{\eta}\right]^{\chi}=H_{P}\left(\widehat{g}^{A}, \widehat{g}^{B}, t, u ; \chi\right)
$$

where $\widehat{g}^{A} \triangleq \widehat{f}^{A}-f, \widehat{g}^{B} \triangleq \widehat{f}^{B}-f$, and

$$
\begin{aligned}
H_{P}\left(\widehat{g}^{A}, \widehat{g}^{B}, t, u ; \chi\right)= & \exp \left\{A_{P}(\chi ; u-t)+C^{A}(\chi ; u-t)\left(\widehat{g}^{A}\right)^{2}+C^{B}(\chi ; u-t)\left(\widehat{g}^{B}\right)^{2}\right. \\
& \left.+2 \widehat{g}^{A} \widehat{g}^{B} C^{A B}(\chi ; u-t)\right\},
\end{aligned}
$$

for certain functions of time $A_{P}, C^{A}, C^{B}$, and $C^{A B}$ that are given in the proof.

Proof: We wish to compute the following expected value:

$$
\widetilde{H}\left(\eta, \widehat{g}^{A}, \widehat{g}^{B}, t, u ; \chi\right)=\mathbb{E}_{\eta, \widehat{g}^{A}, \widehat{g}^{B}}^{P}\left[\eta_{u}\right]^{\chi} .
$$

Under the objective probability measure, the processes $\eta, \widehat{g}^{A}$ and $\widehat{g}^{B}$ obey the following stochastic differential equations:

$$
\begin{aligned}
d \widehat{g}_{t}^{A} & =-\widehat{g}_{t}^{A}\left(\zeta+\frac{\gamma^{A}}{\sigma_{\delta}^{2}}+\frac{\phi \sigma_{s} \sigma_{f}+\gamma^{A}}{\sigma_{s}^{2}}\right) d t+\frac{\gamma^{A}}{\sigma_{\delta}} d Z_{t}^{\delta}+\frac{\phi \sigma_{s} \sigma_{f}+\gamma^{A}}{\sigma_{s}} d Z_{t}^{s}-\sigma_{f} d Z_{t}^{f}, \\
d \widehat{g}_{t}^{B} & =-\widehat{g}_{t}^{B}\left(\zeta+\frac{\gamma^{B}}{\sigma_{\delta}^{2}}+\frac{\gamma^{B}}{\sigma_{s}^{2}}\right) d t+\frac{\gamma^{B}}{\sigma_{\delta}} d Z_{t}^{\delta}+\frac{\gamma^{B}}{\sigma_{s}} d Z_{t}^{s}-\sigma_{f} d Z_{t}^{f} \\
\frac{d \eta_{t}}{\eta_{t}} & =\left(\widehat{g}_{t}^{B}-\widehat{g}_{t}^{A}\right) \widehat{g}_{t}^{B}\left(\frac{1}{\sigma_{\delta}^{2}}+\frac{1}{\sigma_{s}^{2}}\right) d t-\left(\widehat{g}_{t}^{B}-\widehat{g}_{t}^{A}\right)\left(\frac{1}{\sigma_{\delta}} d Z_{t}^{\delta}+\frac{1}{\sigma_{s}} d Z_{t}^{s}\right) .
\end{aligned}
$$


The function $\widetilde{H}\left(\eta, \widehat{g}^{A}, \widehat{g}^{B}, t, u ; \chi\right)$ satisfies the linear PDE:

$$
0 \equiv \mathcal{D} \widetilde{H}\left(\eta, \widehat{g}^{A}, \widehat{g}^{B}, t, u ; \chi\right)+\frac{\partial \widetilde{H}}{\partial t}\left(\eta, \widehat{g}^{A}, \widehat{g}^{B}, t, u ; \chi\right),
$$

with the initial condition $H\left(\eta, \widehat{g}^{A}, \widehat{g}^{B}, t, t ; \chi\right)=\eta^{\chi}$, where $\mathcal{D}$ is the differential generator of $\left(\eta_{t}, \widehat{g}_{t}^{A}, \widehat{g}_{t}^{B}\right)$ under the objective probability measure. Spelling out (A57) using (A54) and (A55), we have:

$$
\begin{aligned}
0= & \frac{\partial \widetilde{H}}{\partial \eta} \eta\left(\widehat{g}_{t}^{B}-\widehat{g}_{t}^{A}\right) \widehat{g}_{t}^{B}\left(\frac{1}{\sigma_{\delta}^{2}}+\frac{1}{\sigma_{s}^{2}}\right)-\frac{\partial \widetilde{H}}{\partial \widehat{g}^{A}}\left(\zeta+\frac{\gamma^{A}}{\sigma_{\delta}^{2}}+\frac{\phi \sigma_{s} \sigma_{f}+\gamma^{A}}{\sigma_{s}^{2}}\right) \widehat{g}^{A} \\
& -\frac{\partial \widetilde{H}}{\partial \widehat{g}^{B}}\left(\zeta+\frac{\gamma^{B}}{\sigma_{\delta}^{2}}+\frac{\gamma^{B}}{\sigma_{s}^{2}}\right) \widehat{g}^{B}+\frac{1}{2} \frac{\partial^{2} \widetilde{H}}{\partial \eta^{2}}\left[\eta\left(\widehat{g}_{t}^{B}-\widehat{g}_{t}^{A}\right)\right]^{2}\left(\frac{1}{\sigma_{\delta}^{2}}+\frac{1}{\sigma_{s}^{2}}\right) \\
& +\frac{1}{2} \frac{\partial^{2} \widetilde{H}}{\partial\left(\widehat{g}^{A}\right)^{2}}\left(\frac{\left(\gamma^{A}\right)^{2}}{\sigma_{\delta}^{2}}+\frac{\left(\phi \sigma_{s} \sigma_{f}+\gamma^{A}\right)^{2}}{\sigma_{s}^{2}}+\sigma_{f}^{2}\right)+\frac{1}{2} \frac{\partial^{2} \widetilde{H}}{\partial\left(\widehat{g}^{B}\right)^{2}}\left(\frac{\left(\gamma^{B}\right)^{2}}{\sigma_{\delta}^{2}}+\frac{\left(\gamma^{B}\right)^{2}}{\sigma_{s}^{2}}+\sigma_{f}^{2}\right) \\
& -\frac{\partial^{2} \widetilde{H}}{\partial \eta \partial \widehat{g}^{A}} \eta\left(\widehat{g}_{t}^{B}-\widehat{g}_{t}^{A}\right)\left(\frac{\gamma^{A}}{\sigma_{\delta}^{2}}+\frac{\phi \sigma_{s} \sigma_{f}+\gamma^{A}}{\sigma_{s}^{2}}\right)-\frac{\partial^{2} \widetilde{H}}{\partial \eta \partial \widehat{g}^{B}} \eta\left(\widehat{g}_{t}^{B}-\widehat{g}_{t}^{A}\right)\left(\frac{\gamma^{B}}{\sigma_{\delta}^{2}}+\frac{\gamma^{B}}{\sigma_{s}^{2}}\right) \\
& +\frac{\partial^{2} \widetilde{H}}{\partial \widehat{g}^{A} \partial \widehat{g}^{B}}\left(\frac{\gamma^{A} \gamma^{B}}{\sigma_{\delta}^{2}}+\frac{\left(\phi \sigma_{s} \sigma_{f}+\gamma^{A}\right) \gamma^{B}}{\sigma_{s}^{2}}+\sigma_{f}^{2}\right)+\frac{\partial \widetilde{H}}{\partial t} .
\end{aligned}
$$

The appropriate solution of this PDE is

$$
\widetilde{H}\left(\eta, \widehat{g}^{A}, \widehat{g}^{B}, t, u ; \chi\right)=\eta^{\chi} \times H_{P}\left(\widehat{g}^{A}, \widehat{g}^{B}, t, u ; \chi\right),
$$

where $H_{P}\left(\widehat{g}^{A}, \widehat{g}^{B}, t, u ; \chi\right)$ is defined in $(\mathrm{A} 52)$ and

$$
\begin{aligned}
A_{P}(u-t)= & \int_{t}^{u}\left[C^{A}(\tau-t)\left(\frac{\left(\gamma^{A}\right)^{2}}{\sigma_{\delta}^{2}}+\frac{\left(\phi \sigma_{s} \sigma_{f}+\gamma^{A}\right)^{2}}{\sigma_{s}^{2}}+\sigma_{f}^{2}\right)+C^{B}(\tau-t)\left(\frac{\left(\gamma^{B}\right)^{2}}{\sigma_{\delta}^{2}}+\frac{\left(\gamma^{B}\right)^{2}}{\sigma_{s}^{2}}+\sigma_{f}^{2}\right)\right. \\
& \left.+2 C^{A B}(\tau-t)\left(\frac{\gamma^{A} \gamma^{B}}{\sigma_{\delta}^{2}}+\frac{\left(\phi \sigma_{s} \sigma_{f}+\gamma^{A}\right) \gamma^{B}}{\sigma_{s}^{2}}+\sigma_{f}^{2}\right)\right] d \tau .
\end{aligned}
$$

The functions of time $C^{A}, C^{A B}$ and $C^{B}$ are obtained as the elements of the matrix $Z=\left(\begin{array}{cc}C^{A} & C^{A B} \\ C^{A B} & C^{B}\end{array}\right)$, itself defined as follows. Let matrices $X(u-t)$ and $Y(u-t)$ be the unique solution of the linear Cauchy problem

$$
\left\{\begin{array}{cc}
\dot{X}=Q^{11} X+Q^{12} Y, & X(0)=I, \\
\dot{Y}=Q^{21} X+Q^{22} Y, & Y(0)=0,
\end{array}\right.
$$

where $I$ is the identity $2 \times 2$ matrix. Then $Z(u-t)=Y(u-t)[X(u-t)]^{-1}$. The coefficients in (A61) are given by:

$$
\begin{aligned}
& Q^{21}=\left(\begin{array}{cc}
\frac{1}{2} \chi(\chi-1)\left(\frac{1}{\sigma_{\delta}^{2}}+\frac{1}{\sigma_{s}^{2}}\right) & -\frac{1}{2} \chi^{2}\left(\frac{1}{\sigma_{\delta}^{2}}+\frac{1}{\sigma_{s}^{2}}\right) \\
-\frac{1}{2} \chi^{2}\left(\frac{1}{\sigma_{\delta}^{2}}+\frac{1}{\sigma_{s}^{2}}\right) & \frac{1}{2} \chi(\chi+1)\left(\frac{1}{\sigma_{\delta}^{2}}+\frac{1}{\sigma_{s}^{2}}\right)
\end{array}\right), \\
& Q^{11}=-\left(Q^{22}\right)^{\top}=\left(\begin{array}{cc}
\zeta+(1-\chi)\left(\frac{\gamma^{A}}{\sigma_{\delta}^{2}}+\frac{\phi \sigma_{s} \sigma_{f}+\gamma^{A}}{\sigma_{s}^{2}}\right) & \chi\left(\frac{\gamma^{A}}{\sigma_{\delta}^{2}}+\frac{\phi \sigma_{s} \sigma_{f}+\gamma^{A}}{\sigma_{s}^{2}}\right) \\
-\chi\left(\frac{\gamma^{B}}{\sigma_{\delta}^{2}}+\frac{\gamma^{B}}{\sigma_{s}^{2}}\right) & \zeta+(1+\chi)\left(\frac{\gamma^{B}}{\sigma_{\delta}^{2}}+\frac{\gamma^{B}}{\sigma_{s}^{2}}\right)
\end{array}\right), \\
& Q^{12}=\left(\begin{array}{cc}
-2\left(\frac{\left(\gamma^{A}\right)^{2}}{\sigma_{\delta}^{2}}+\frac{\left(\phi \sigma_{s} \sigma_{f}+\gamma^{A}\right)^{2}}{\sigma_{s}^{2}}+\sigma_{f}^{2}\right) & -2\left(\frac{\gamma^{A} \gamma^{B}}{\sigma_{\delta}^{2}}+\frac{\left(\phi \sigma_{s} \sigma_{f}+\gamma^{A}\right) \gamma^{B}}{\sigma_{s}^{2}}+\sigma_{f}^{2}\right. \\
-2\left(\frac{\gamma^{A} \gamma^{B}}{\sigma_{\delta}^{2}}+\frac{\left(\phi \sigma_{s} \sigma_{f}+\gamma^{A}\right) \gamma^{B}}{\sigma_{s}^{2}}+\sigma_{f}^{2}\right) & -2\left(\frac{\left(\gamma^{B}\right)^{2}}{\sigma_{\delta}^{2}}+\frac{\left(\gamma^{B}\right)^{2}}{\sigma_{s}^{2}}+\sigma_{f}^{2}\right)
\end{array}\right),
\end{aligned}
$$

which completes the proof. 


\section{B The wealth and price functions}

Knowing the characteristic function (A1) from Appendix A, the single-maturity claims prices $\mathbb{E}_{\eta, \widehat{f}^{B}, \widehat{g}}\left[\frac{\xi_{u}^{B}}{\xi_{t}^{B}}\right]$, $\mathbb{E}_{\eta, \hat{f}^{B}, \widehat{g}}\left[\frac{\xi_{u}^{B}}{\xi_{t}^{B}} \frac{\delta_{u}}{\delta_{t}}\right]$ and $\mathbb{E}_{\eta, \widehat{f}^{B}, \widehat{g}}\left[\frac{\xi_{u}^{B}}{\xi_{t}^{B}} \frac{c_{u}^{B}}{c_{t}^{B}}\right]$ can be obtained by one of two methods. One is applicable only if $1-\alpha \in \mathbb{N}$. Then the bracket $\left[\left(\frac{\eta_{u}}{\lambda^{A}}\right)^{\frac{1}{1-\alpha}}+\left(\frac{1}{\lambda^{B}}\right)^{\frac{1}{1-\alpha}}\right]^{1-\alpha}$ can be expanded into an exact finite series by virtue of the binomial formula as in Equation (42) of the text. The overall calculation in this case is greatly simplified. It leads to the prices of single-maturity claims (43), (44) and (48). ${ }^{51}$ The second method is general in that it applies for any value of risk aversion. This method is the inverse Fourier transform, for which the formulae are given below and which can be computed by means of the Fast Fourier Transform:

$$
\begin{aligned}
\mathbb{E}_{\eta, \widehat{f}^{B}, \widehat{g}}^{B}\left[\frac{\xi_{u}^{B}}{\xi_{t}^{B}}\right]= & e^{-\rho(u-t)} H_{f}\left(\widehat{f}^{B}, t, u ; \alpha-1\right) \\
& \times \int_{0}^{\infty}\left(\frac{1-\omega(\eta)}{1-\omega(\widetilde{\eta})}\right)^{1-\alpha}\left[\frac{1}{2 \pi} \int_{-\infty}^{+\infty}\left(\frac{\widetilde{\eta}}{\eta}\right)^{-i \chi} H_{g}(\widehat{g}, t, u ; \alpha-1, i \chi) d \chi\right] \frac{d \widetilde{\eta}}{\widetilde{\eta}}, \\
\mathbb{E}_{\eta, \widehat{f}^{B}, \widehat{g}}^{B}\left[\frac{\xi_{u}^{B}}{\xi_{t}^{B}} \frac{\delta_{u}}{\delta_{t}}\right]= & e^{-\rho(u-t)} H_{f}\left(\widehat{f}^{B}, t, u ; \alpha\right) \\
& \times \int_{0}^{\infty}\left(\frac{1-\omega(\eta)}{1-\omega(\widetilde{\eta})}\right)^{1-\alpha}\left[\frac{1}{2 \pi} \int_{-\infty}^{+\infty}\left(\frac{\widetilde{\eta}}{\eta}\right)^{-i \chi} H_{g}(\widehat{g}, t, u ; \alpha, i \chi) d \chi\right] \frac{d \widetilde{\eta}}{\widetilde{\eta}}, \\
\mathbb{E}_{\eta, \widehat{f}^{B}, \widehat{g}}^{B}\left[\frac{\xi_{u}^{B}}{\xi_{t}^{B}} \frac{c_{u}^{B}}{c_{t}^{B}}\right]= & e^{-\rho(u-t)} H_{f}\left(\widehat{f}^{B}, t, u ; \alpha\right) \\
& \times \int_{0}^{\infty}\left(\frac{1-\omega(\eta)}{1-\omega(\widetilde{\eta})}\right)^{-\alpha}\left[\frac{1}{2 \pi} \int_{-\infty}^{+\infty}\left(\frac{\widetilde{\eta}}{\eta}\right)^{-i \chi} H_{g}(\widehat{g}, t, u ; \alpha, i \chi) d \chi\right] \frac{d \widetilde{\eta}}{\widetilde{\eta}} .
\end{aligned}
$$

Similarly, the share of consumption of Group $A$ under the objective probability measure is:

$$
\mathbb{E}_{\eta, \widehat{g}^{A}, \widehat{g}^{B}}^{P}\left[\omega\left(\eta_{u}\right)\right]=\int_{0}^{\infty} \omega(\widetilde{\eta})\left[\frac{1}{2 \pi} \int_{-\infty}^{+\infty}\left(\frac{\widetilde{\eta}}{\eta}\right)^{-i \chi} H_{P}\left(\widehat{g}^{A}, \widehat{g}^{B}, t, u ; i \chi\right) d \chi\right] \frac{d \widetilde{\eta}}{\widetilde{\eta}}
$$

Finally, because the prices of perpetual claims (46) and (45) involve time integrals of (B2) and (B1) (or (43) and(44)) and these integrals have an infinite upper bound, we need to check for convergence. The conditions for convergence of prices are derived in the theorem below

Theorem 3 The growth conditions for the price of equity, $F$, and the price of the bond, $P$, to be well defined are, respectively:

$$
G(\alpha)<\rho \text { and } G(\alpha-1)<\rho,
$$

where

$$
G(\varepsilon)=\varepsilon \bar{f}+\frac{1}{2} \varepsilon(\varepsilon-1) \sigma_{\delta}^{2}+\frac{\varepsilon^{2} \sigma_{f}^{2}}{2 \zeta^{2}}
$$

Proof: Since for $u \geq t$ and $\chi \in[0,1],\left|\frac{b-q}{q+b} e^{-q(u-t)}\right|<1$, we can take Taylor series in (A27):

$$
\begin{aligned}
B(u-t) & =\frac{\sum_{i=1}^{5} \vartheta_{i} e^{v_{i}(u-t)}}{(q+b)\left(1-\frac{b-q}{q+b} e^{-2 q(u-t)}\right)}=\frac{\sum_{i=1}^{5} \vartheta_{i} e^{v_{i}(u-t)}}{(q+b)} \sum_{j=0}^{\infty}\left[\frac{b-q}{q+b} e^{-2 q(u-t)}\right]^{j}, \\
& =\sum_{j=0}^{\infty}\left[h_{j}^{I} e^{-j q(u-t)}+h_{j}^{I I} e^{-(j q+\zeta)(u-t)}\right],
\end{aligned}
$$

\footnotetext{
${ }^{51}$ The $\chi$ argument belongs to $[0,1]$ allowing us to apply Lemma 1 to conclude that $H_{g}$ is well defined.
} 
where $h_{j}^{I}$, and $h_{j}^{I I}$ are certain functions of $\chi$ (and independent of time) such that the series in (B7) is uniformly convergent.

Define

$$
\Pi\left(\widehat{f}^{B}, \widehat{g}, t, u ; \varepsilon, \chi\right)=e^{-\rho(u-t)} H_{f}\left(\widehat{f}^{B}, t, u ; \varepsilon\right) H_{g}(\widehat{g}, t, u ; \varepsilon, \chi) .
$$

Since in (A4) $A_{1}, A_{2}, C \leq 0$, we have $\Pi \leq \bar{\Pi}$, where

$$
\bar{\Pi}\left(\widehat{f}^{B}, \widehat{g}, t, u ; \varepsilon, \chi\right)=e^{-\rho(u-t)} H_{f}\left(\widehat{f}^{B}, t, u ; \varepsilon\right) \exp [\varepsilon \widehat{g} B(\chi ; u-t)] .
$$

Substituting (B7)and (A3), we find that the function $\bar{\Pi}$ may be written as:

$$
\begin{aligned}
& \bar{\Pi}\left(\widehat{f}^{B}, \widehat{g}, t, u ; \varepsilon, \chi\right)=\pi_{0}\left(\widehat{f}^{B}, \widehat{g} ; \varepsilon, \chi\right) \exp \left\{[G(\varepsilon)-\rho](u-t)+\pi_{1}(\varepsilon) e^{-2 \zeta(u-t)}\right. \\
& \left.\quad+\sum_{j=0}^{\infty}\left[\pi_{j}^{I}(\widehat{g} ; \varepsilon, \chi) e^{-(j+1) q(u-t)}+\pi_{j}^{I I}\left(\widehat{f}^{B}, \widehat{g} ; \varepsilon, \chi\right) e^{-(j q+\zeta)(u-t)}\right]\right\},
\end{aligned}
$$

where the series converges uniformly.

Following the line of argument in Brennan and Xia (2001, Theorem 6) ${ }^{52}$ one can easily show that, when $\chi \in[0,1]$, the integrals

$$
\int_{t}^{\infty} \bar{\Pi}\left(\widehat{f}^{B}, \widehat{g}, t, u ; \varepsilon, \chi\right) d u
$$

and

$$
\int_{t}^{\infty} \Pi\left(\hat{f}^{B}, \widehat{g}, t, u ; \varepsilon, 0\right) d u=\int_{t}^{\infty} e^{-\rho(u-t)} H_{f}\left(\widehat{f}^{B}, t, u ; \varepsilon\right) d u
$$

are finite if and only if $G(\varepsilon)-\rho<0$.

Assume first that $1-\alpha \in \mathbb{N}$. Then (46) and (45) imply that the prices are well defined as long as the integral $\int_{t}^{\infty} \Pi\left(\hat{f}^{B}, \widehat{g}, t, u ; \varepsilon, \chi\right) d u$ is convergent for every $\chi \in J=\left\{\frac{j}{1-\alpha}\right\}_{j=0}^{1-\alpha}$ and when $\varepsilon=\alpha$ for the stock and $\varepsilon=\alpha-1$. Therefore, convergence of integral (B12) is a necessary condition (since $\chi=0 \in J$ ) and convergence of integral (B11) is a sufficient condition (since $\Pi \leq \bar{\Pi}$ ) for the prices to be well defined. It only remains to notice that, for each price, these conditions are identical and are given by (B5).

Now, let $\alpha$ be any real number such that $1-\alpha>0$. Observe that when $y>0$, the function $f(x)=\left(1+y^{1 / x}\right)^{x}$ increases with $x>0$ :

$$
\begin{aligned}
f^{\prime}(x) & =\frac{1}{x}\left(1+y^{1 / x}\right)^{x-1}\left[x\left(1+y^{1 / x}\right) \ln \left(1+y^{1 / x}\right)-y^{1 / x} \ln y\right] \\
& >\frac{1}{x}\left(1+y^{1 / x}\right)^{x-1}\left[x y^{1 / x} \ln \left(y^{1 / x}\right)-y^{1 / x} \ln y\right]=0 .
\end{aligned}
$$

Therefore,

$$
\frac{1}{\lambda^{B}}<\left[\left(\frac{\eta_{u}}{\lambda^{A}}\right)^{\frac{1}{1-\alpha}}+\left(\frac{1}{\lambda^{B}}\right)^{\frac{1}{1-\alpha}}\right]^{1-\alpha} \leq\left[\left(\frac{\eta_{u}}{\lambda^{A}}\right)^{\frac{1}{1-[\alpha]}}+\left(\frac{1}{\lambda^{B}}\right)^{\frac{1}{1-[\alpha]}}\right]^{1-[\alpha]} .
$$

Replacing the bracket $\left[\left(\frac{\eta_{u}}{\lambda^{A}}\right)^{\frac{1}{1-\alpha}}+\left(\frac{1}{\lambda^{B}}\right)^{\frac{1}{1-\alpha}}\right]^{1-\alpha}$ with its lower and upper bounds (B14) in the expressions (45) and (46) for securities market prices and applying the above results for the case of $1-\alpha \in \mathbb{N}$, we obtain necessary (when the lower bound is substituted; $\chi=0$ ) and sufficient (when the upper bound is substituted; $\chi=\frac{j}{1-[\alpha]}, j=0, \ldots, 1-[\alpha]$ ) conditions for the prices to be well defined. The fact that, for each price, the necessary and sufficient conditions are identical and are given by (B5) completes the proof.

\footnotetext{
${ }^{52}$ To show that for integral (B11), one should additionally note that, since $q, \zeta>0$ and the series in (B10) is uniformly convergent, it tends to zero when $u \rightarrow \infty$.
} 


\section{References}

Abel, A. B., 1990, "Asset Prices under Habit Formation and Catching Up with the Joneses," American Economic Review, 80, 38-42.

Alchian, A., 1950, "Uncertainty, Evolution and Economic Theory," The Journal of Political Economy, $58,211-221$.

Allen, F., S. Morris, and H. S. Shin, 2004, "Beauty Contests and Iterated Expectations in Asset Markets," Working paper, University of Pennsylvania.

Bansal, R., and A. Yaron, 2004, "Risks for the Long Run: A Potential Resolution of Asset Pricing Puzzles," Journal of Finance, 59, 1481-151.

Barberis, N., A. Shleifer, and R. Vishny, 1998, "A Model of Investor Sentiment," Journal of Financial Economics, 49, 307-43.

Barsky, R. B., and J. B. De Long, 1993, "Why Does the Stock Market Fluctuate?," Quarterly Journal of Economics, 108, 291-311.

Basak, S., 2004, "Asset Pricing with Heterogeneous Beliefs," forthcoming in Journal of Banking and Finance.

Berrada, T., 2004, "Bounded Rationality and Asset Pricing," Working paper, HEC Montreal.

Biais, B., and P. Bossaerts, 1998, "Asset Prices and Trading Volume in a Beauty Contest," Review of Economic Studies, 65, 307-40.

Brennan, M. J., and Y. Xia, 2001, "Stock Price Volatility and Equity Premium," Journal of Monetary Economics, 47, 249-83.

Bullard, J., and J. Duffy, 1998, "Learning and Excess Volatility," Working paper, Federal Reserve Bank of Saint-Louis.

Buraschi, A., and A. Jiltsov, 2005, "Model Uncertainty and Option Markets with Heterogeneous Agents," forthcoming in Journal of Finance.

Campbell, J. Y., and J. H. Cochrane, 1999, "By Force of Habit: A Consumption-Based Explanation of Aggregate Stock Market Behavior," Journal of Political Economy, 107, 205-51.

Campbell, J. Y., and R. J. Shiller, 1988a, "The Dividend-Price Ratio and Expectations of Future Dividends and Discount Factors," Review of Financial Studies, 1, 195-227.

Campbell, J. Y., and R. J. Shiller, 1988b, "Stock Prices, Earnings, and Expected Dividends," Journal of Finance, 43, 661-76.

Cao, H. H., and H. Ou-Yang, 2005, "Bubbles and Panics in a Frictionless Market with Heterogeneous Expectations," Working paper, Cheung Kong Graduate School of Business.

Cecchetti, S. G., P.-s. Lam, and N. C. Mark, 2000, "Asset Pricing with Distorted Beliefs: Are Equity Returns Too Good to Be True?," American Economic Review, 90, 787-805.

Cochrane, J. H., 1991, "Volatility Tests and Efficient Markets: Review Essay," Journal of Monetary Economics, 27, 463-85.

Cochrane, J. H., 2001, Asset Pricing, Princeton University Press.

Constantinides, G. M., 1990, "Habit Formation: A Resolution of the Equity Premium Puzzle," Journal of Political Economy, 98, 519-43.

Cox, J. C., and C.-f. Huang, 1989, "Optimal Consumption and Portfolio Policies When Asset Prices Follow a Diffusion Process," Journal of Economic Theory, 49, 33-83.

Daniel, K., D. Hirshleifer, and A. Subrahmanyam, 1998, "Investor Psychology and Security Market Under- and Overreactions," Journal of Finance, 53, 1839-85.

David, A., 2004, "Heterogeneous Beliefs, Trading Risk, and the Equity Risk Premium," Working paper, Washington University.

David, A., and P. Veronesi, 2002, "Option Prices with Uncertain Fundamentals: Theory and Evidence on the Dynamics of Implied Volatilities," Working paper, University of Chicago. 
De Long, J. B., A. Shleifer, L. H. Summers, and R. J. Waldmann, 1990a, "Noise Trader Risk in Financial Markets," Journal of Political Economy, 98, 703-38.

De Long, J. B., A. Shleifer, L. H. Summers, and R. J. Waldmann, 1990b, "Positive Feedback Investment Strategies and Destabilizing Rational Speculation," Journal of Finance, 45, 379-95.

De Long, J. B., A. Shleifer, L. H. Summers, and R. J. Waldmann, 1991, "The Survival of Noise Traders in Financial Markets," Journal of Business, 64, 1-20.

Detemple, J., and S. Murthy, 1994, "Intertemporal Asset Pricing with Heterogenous Beliefs," Journal of Economic Theory, 62, 294-320.

Duffie, D., N. Garleanu, and L. H. Pedersen, 2002, "Securities Lending, Shorting, and Pricing," Journal of Financial Economics, 66, 307-39.

Feldman, D., 2005, "Incomplete Information Equilibria: Separation Theorems and Other Myths," Working paper, The University of New South Wales.

Flavin, M. A., 1983, "Excess Volatility in the Financial Markets: A Reassessment of the Empirical Evidence," Journal of Political Economy, 91, 929-56.

Friedman, M., 1953, Essays in Positive Economics, University of Chicago Press, Chicago, IL.

Froot, K. A., and J. Frankel, 1989, "Forward Discount Bias: Is it an Exchange Risk Premium?," Quarterly Journal of Economics, 104, 139-161.

Gallmeyer, M., 2000, "Beliefs and Volatility," Working paper, Carnegie Mellon University.

Gallmeyer, M., and B. Hollifield, 2004, "An Examination of Heterogeneous Beliefs with a Short Sale Constraint," Working paper, Carnegie-Mellon University.

Gollier, C., 2005, "Who Should We Believe? Collective Risk-Taking Decisions with Heterogeneous Beliefs," Working paper, University of Toulouse.

Grossman, S. J., and J. E. Stiglitz, 1980, "On the Impossibility of Informationally Efficient Markets," American Economic Review, 70, 393-408.

Hansen, L. P., J. C. Heaton, and N. Li, 2005, "Consumption Strikes Back? Measuring Long Run Risk," Working paper, University of Chicago.

Harris, M., and A. Raviv, 1993, "Differences of Opinion Make a Horse Race," Review of Financial Studies, 6, 473-506.

Harrison, J. M., and D. M. Kreps, 1978, "Speculative Investor Behavior in a Stock Market with Heterogeneous Expectations," Quarterly Journal of Economics, 92, 323-336.

Hellwig, M. F., 1980, "On the Aggregation of Information in Complete Markets," Journal of Economic Theory, 26, 279-312.

Heston, S. L., 1993, "A Closed-Form Solution for Options with Stochastic Volatility with Applications to Bond and Currency Options," Review of Financial Studies, 6, 327-43.

Hong, H., and J. C. Stein, 1999, "A Unified Theory of Underreaction, Momentum Trading, and Overreaction in Asset Markets," Journal of Finance, 54, 2143-84.

Jouini, E., and C. Napp, 2003, "Consensus Consumer and Intertemporal Asset Pricing with Heterogeneous Beliefs," Working paper, CEREMADE Universite Paris 9-Dauphine.

Kandel, E., and N. D. Pearson, 1995, "Differential Interpretation of Public Signals and Trade in Speculative Markets," The Journal of Political Economy, 103, 831-872.

Karatzas, I., J. Lehoczky, and S. Shreve, 1987, "Optimal Portfolio and Consumption Decisions for a 'Small Investor' on a Finite Horizon," SIAM Journal of Control and Optimization, 25, 1157-1186.

Kim, T. S., and E. Omberg, 1996, "Dynamic Nonmyopic Portfolio Behavior," Review of Financial Studies, 9, 141-61.

Kleidon, A. W., 1986, "Variance Bounds Tests and Stock Price Valuation Models," Journal of Political Economy, 94, 953-1001.

Kogan, L., S. Ross, J. Wang, and M. Westerfield, 2003, "The Price Impact and Survival of Irrational Traders," Working paper, MIT. 
LeRoy, S. F., and R. D. Porter, 1981, "The Present-Value Relation: Tests Based on Implied Variance Bounds," Econometrica, 49, 555-74.

Lintner, J., 1969, "The Aggregation of Investor's Diverse Judgments and Preferences in Purely Competitive Security Markets," Journal of Financial and Quantitative Analysis, 4, 347-400.

Lipster, R. S., and A. N. Shiryaev, 2001, Statistics of Random Processes II, Applications, Springer Verlag, second edn.

Loewenstein, M., and G. A. Willard, 2005, "The Limits of Investor Behavior," Working paper, University of Maryland.

Menzly, L., T. Santos, and P. Veronesi, 2004, "Understanding Predictability," Journal of Political Economy, 112, 1-47.

Merton, R. C., 1971, "Optimum Consumption and Portfolio Rules in a Continuous Time Model," Journal of Economic Theory, 3, 373-413.

Merton, R. C., 1973, "The Theory of Rational Option Pricing," Bell Journal of Economics and Management Science, 4.

Sandroni, A., 2000, "Do Markets Favor Agents Able to Make Accurate Predictions?," Econometrica, 68, $1303-1341$.

Scheinkman, J. A., and W. Xiong, 2003, "Overconfidence and Speculative Bubbles," Journal of Political Economy, 111, 1183-1219.

Shefrin, H., 2005, A Behavioral Approach to Asset Pricing, Academic Press Inc., London.

Shiller, R. J., 1981, "Do Stock Prices Move Too Much to be Justified by Subsequent Changes in Dividends?," American Economic Review, 71, 421-36.

Timmermann, A., 1996, "Excess Volatility and Predictability of Stock Prices in Autoregressive Dividend Models with Learning," Review of Economic Studies, 63, 523-57.

Timmermann, A. G., 1993, "How Learning in Financial Markets Generates Excess Volatility and Predictability in Stock Prices," Quarterly Journal of Economics, 108, 1135-45.

Veronesi, P., 1999, "Stock Market Overreaction to Bad News in Good Times: A Rational Expectations Equilibrium Model," Review of Financial Studies, 12, 975-1007.

Wang, J., 1993, "A Model of Intertemporal Asset Prices under Asymmetric Information," Review of Economic Studies, 60, 249-82.

West, K. D., 1988a, "Bubbles, Fads and Stock Price Volatility Tests: A Partial Evaluation," Journal of Finance, 43, 639-56.

West, K. D., 1988b, "Dividend Innovations and Stock Price Volatility," Econometrica, 56, 37-61.

Williams, J. T., 1977, "Capital Asset Prices with Heterogeneous Beliefs," Journal of Financial Economics, 5, 219-39.

Yan, H., 2004, "Natural Selection in Financial Markets: Does It Work?," Working paper, London Business School.

Ziegler, A., 2000, "Optimal Portfolio Choice under Heterogeneous Beliefs," European Finance Review, 4, 1-19. 